\title{
Gravitational radiation detection with laser interferometry
}

\author{
Rana X. Adhikari* \\ Division of Physics, Math, and Astronomy, California Institute of Technology, Pasadena, \\ California 91125, USA
}

(published 21 February 2014)

\begin{abstract}
Gravitational-wave detection has been pursued relentlessly for over 40 years. With the imminent operation of a new generation of laser interferometers, it is expected that detections will become a common occurrence. The research into more ambitious detectors promises to allow the field to move beyond detection and into the realm of precision science using gravitational radiation. In this article, the state of art for the detectors is reviewed and an outlook for the coming decades is described.
\end{abstract}

DOI: $10.1103 /$ RevModPhys.86.121

PACS numbers: 04.30.- w, 04.80.Nn, 95.55.Ym, 07.60.Ly

\section{CONTENTS}

I. Introduction

II. Gravitational Waves

A. Response of interferometer to space-time strain

B. Brief overview of sources

1. Pulsars

2. Transients

3. Cosmic background radiation

III. Alternatives to Interferometric Detection
A. Acoustic detectors
B. Pulsar timing
C. Artificial satellite timing
D. Polarization of the microwave background

IV. Fundamentals of Interferometric Detectors

A. Delay lines versus Fabry-Pérot resonator 125

B. Power recycling

C. Signal recycling and extraction

V. Sensitivity Limits of Laser Interferometers

A. Phase noise

1. Quantum vacuum fluctuations

2. Scattering from residual gas

3. Backscatter

B. Displacement noise

1. Mirror thermal noise

2. Suspension thermal noise

3. Seismic vibrations

4. Newtonian gravity noise

5. Electromagnetic coupling

VI. First Generation Detectors

A. Excess optical loss

B. Optical cross coupling

1. Fluctuations of the light

2. Local oscillator phase noise

3. Motion of auxiliary mirrors

C. Low-frequency mirror motion

1. Seismic amplification

2. Noise from damping

D. Nonlinear noise generation

1. Bilinear angle to length conversion
2. Actuator nonlinearities

133

E. Thermal distortions 133

VII. Second Generation Detectors

134

A. Monolithic silica suspensions $\quad 134$

B. Mirror metrology 134

C. Dual recycling 135

D. High-power optomechanics 136

1. Angular instabilities 136

2. Parametric instabilities 137

E. Low-frequency seismic isolation $\quad 138$

VIII. Third Generation Detectors 138

A. Quantum nondemolition 139

1. Frequency dependent squeezed state injection 139

2. Frequency dependent readout quadrature $\quad 140$

3. QND observable readout 140

4. Optical losses and QND 140

B. Circumventing mirror thermal noise 140

1. Non-Gaussian beam shapes 140

2. Heteroepitaxial Bragg mirrors 141

C. Newtonian gravity noise subtraction 141

D. Beyond silica suspensions 142

1. Silicon suspensions and cryogenics 142

2. Electromagnetic suspensions 143

IX. Low-frequency Detectors 143

A. Interferometers in space 143

1. eLISA 143

2. DECIGO and BBO 144

B. Low-frequency terrestrial detectors 144

1. Torsion bar antenna 144

2. Atom interferometers 145

X. Conclusion 145

Acknowledgments $\quad 145$

$\begin{array}{ll}\text { References } & 145\end{array}$

\section{INTRODUCTION}

Nearly a century ago, Einstein predicted the existence of gravitational radiation as a consequence of his general theory of relativity (GR) (Einstein, 1916, 1918; Einstein and Engel, 1997). For the next several decades, the existence and properties of gravitational radiation were hotly contested within the theoretical community but remained out of observational

*rana@caltech.edu 
reach. In 1974, Hulse and Taylor (Taylor, Fowler, and McCulloch, 1979) discovered a pulsar in a binary neutron star system. They soon realized that this system serves as an excellent laboratory to test GR. The decrease in the orbital energy of the binary system was found to match the theoretical predictions. During the following decades several other binary pulsars with orbital periods of less than a day have been discovered and the combined data show that the measured energy loss matches well with the calculated loss due to the emission of gravitational radiation. Gravitational waves (GWs) are real.

Modern efforts to detect gravitational radiation on the Earth focus on the use of laser interferometry (Weiss, 1972). Laboratory scale research throughout the last several decades of the 20th century led to the construction of a worldwide network of kilometer scale interferometers (Abramovici et al., 1992; Lück et al., 2006; Acernese et al., 2008; Tatsumi, 2008; Abbott et al., 2009c).

Several excellent monographs (Giazotto, 1989; Saulson, 1994; Barish and Weiss, 1999; Weiss, 1999; Aufmuth and Danzmann, 2005; Braginsky, 2008; Freise and Strain, 2010; Cella and Giazotto, 2011; Pitkin et al., 2011) have been written on the techniques of gravitational-wave detection by laser interferometry. In this review, we discuss the current state of gravitational-wave detectors, describing in detail the fundamental limits to their astrophysical reach, and then present prospects for the future.

\section{GRAVITATIONAL WAVES}

In the weak-field approximation of general relativity, the space-time metric $g_{\mu \nu}$, can be described as (Misner, Thorne, and Wheeler, 1973)

$$
g_{\mu \nu} \simeq \eta_{\mu \nu}+h_{\mu \nu},
$$

where $\eta_{\mu \nu}$ is the Minkowski metric describing flat space and $h_{\mu \nu}$ is the perturbation to the metric due to the gravitational wave. In the transverse-traceless gauge, this can be understood as a strain in space-time:

$$
h_{\mu \nu}(z, t)=\left(\begin{array}{cccc}
0 & 0 & 0 & 0 \\
0 & -h_{+} & h_{\times} & 0 \\
0 & h_{\times} & h_{+} & 0 \\
0 & 0 & 0 & 0
\end{array}\right),
$$

where the two independent polarizations of the wave have amplitudes $h_{+}$and $h_{\times}$, respectively.

\section{A. Response of interferometer to space-time strain}

In order to relate this perturbed metric with laboratory observables, we can examine how some precision measurement apparatus will respond to such a strain. To illustrate this we set up two free masses, one located at the origin and one located a distance $x=L$ from the origin. We measure the separation between these two masses by sending a laser beam from the origin to bounce off of the far mass and measure the phase of the return beam relative to the source. The accumulated round-trip phase is

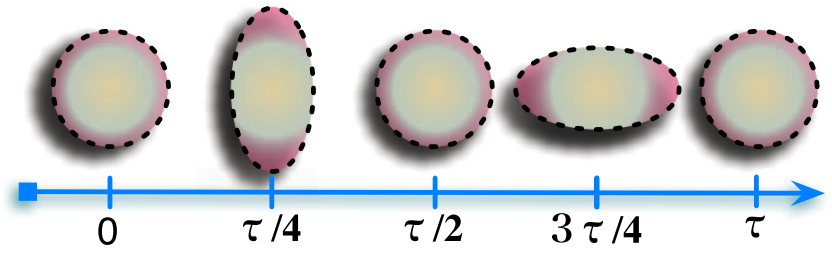

FIG. 1 (color online). Exaggerated example of the effect of a GW on a ring of test particles. The GW is coming from above, is "plus" polarized, and has a period $\tau$. As the wave passes, the ring is alternately stretched and compressed. This quadrupolar strain pattern matches well to the geometry of a Michelson interferometer.

$$
\Phi_{\mathrm{rt}}\left(t_{\mathrm{rt}}\right)=\int_{0}^{t_{\mathrm{rt}}} 2 \pi \nu d t
$$

where $t_{\mathrm{rt}}$ is the time it takes for the light to make one roundtrip and $\nu$ is the frequency of the light. In the absence of gravitational radiation, we can do the integral by changing it into an integral over length. To do this we use the flat space metric $\eta_{\mu \nu}$ to relate space and time for light $\left(t_{\mathrm{rt}}=2 L / c\right.$ and $d t=d x / c)$.

In the presence of a gravitational wave, we instead use Eq. (1) to calculate the space-time interval; the perturbed round-trip phase is

$$
\Phi_{\mathrm{rt}}\left(t_{\mathrm{rt}}\right)=2 \frac{2 \pi \nu}{c} \int_{0}^{L} \sqrt{\left|g_{x x}\right|} d x \simeq 2\left(1-h_{+} / 2\right) \frac{2 \pi L}{\lambda}
$$

in the case of a "plus" oriented wave with a period much longer than the round-trip light travel time. Repeating this integral, but doing the integration now along the $y$ axis, we get that $\Phi_{\mathrm{rt}} \simeq 2\left(1+h_{+} / 2\right)(2 \pi L / \lambda)$. The difference in the phase shift between the two arms is then $\Delta \Phi \simeq 2 h_{+}(2 \pi L / \lambda)$.

Interpreting the phase shifts as length variations means that the apparent length of each arm is stretched and compressed as the gravitational wave passes. A diagram of this is shown in Fig. 1. The length change is proportional to the original distance between the masses,

$$
\frac{\Delta L}{L}=\frac{1}{2} h_{+}
$$

which is why a gravitational wave is said to cause a strain in space. In contrast, the term "gravity wave" is usually used to refer to waves in fluids or solids where the restoring force is due to gravity.

The strain along the interferometer arms for a gravitational wave from an arbitrary direction (in spherical coordinates centered on the detector) is (Christensen, 1992)

$$
\begin{aligned}
& h_{x x}=-\cos \theta \sin 2 \phi h_{\times}+\left(\cos ^{2} \theta \cos ^{2} \phi-\sin ^{2} \phi\right) h_{+}, \\
& h_{y y}=\cos \theta \sin 2 \phi h_{\times}+\left(\cos ^{2} \theta \sin ^{2} \phi-\cos ^{2} \phi\right) h_{+} .
\end{aligned}
$$

The interferometer response in the low-frequency approximation (time scales much longer than the one way light travel time) is proportional to $\left|h_{y y}-h_{x x}\right|$. Figure 2 shows this dc response for + waves, for $\times$ waves, and for unpolarized waves (a quadrature sum of the two cases). In the coordinate system used in these plots, the interferometer is located at the origin with the arms parallel to the $x$ and $y$ axes. 

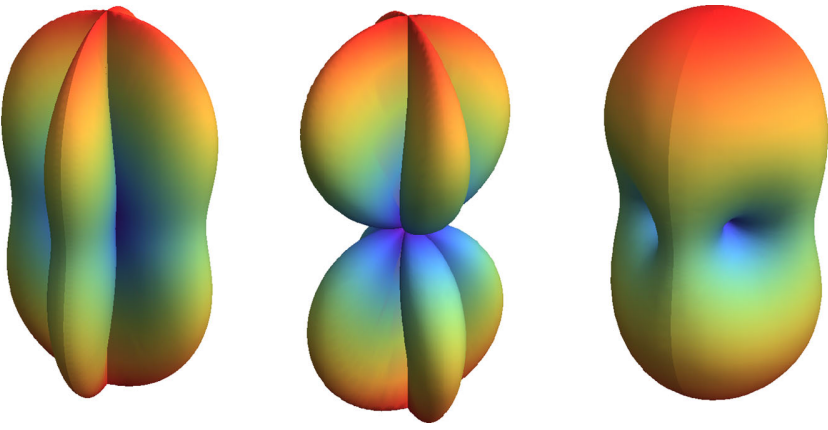

FIG. 2 (color online). Interferometer antenna response for $(+)$ polarization (left), $(\times)$ polarization (middle), and unpolarized waves (right).

\section{B. Brief overview of sources}

All terrestrial detectors of gravitational waves are focused roughly on the audio frequency band due to technological limits of the detectors and probable source characteristics. In order to verify all of the properties of the waves, one would like to follow in the footsteps of Heinrich Hertz by generating and then detecting the gravitational waves. However, due to the relatively high rigidity of space-time, it is not feasible to generate measurable amounts of gravitational radiation in the laboratory (Romero and Dehnen, 1981) by conventional means or even through the use of nuclear explosives arranged to produce quadrupolar mass-energy accelerations (Chapline, Nuckolls, and Wood, 1974). Therefore, we look to astrophysical and cosmological sources to provide the radiation. In this way, the hunt for gravitational radiation leads to the development of a new branch of astronomy. Previous overviews (Hawking and Israel, 1989; Cutler and Thorne, 2002) covered the list of known sources as well as describing the astrophysical and cosmological science that can be extracted from them (Sathyaprakash and Schutz, 2009).

\section{Pulsars}

One of the earliest predicted sources of gravitational radiation were the recently discovered pulsars (Hewish et al., 1968). The extremely stable period of pulsation of these rotating neutron stars tells us that the energy lost to gravitational radiation must be small (Ipser, 1971) at best. The compensating factor that makes detection a possibility is the periodic nature of the signal; after correcting for the Doppler modulations from the detector motions relative to the source (Brady et al., 1998; Abbott et al., 2009b), one can improve the signal-to-noise ratio by the square root of the integration time.

Observations (Chakrabarty et al., 2003) of a "speed limit" for pulsars seem to support the theory (Bildsten, 1998) that gravitational radiation works to brake the spin of the fastest pulsars before they are ripped apart by their relativistic spins. Expectations from neutron star models indicate that the ellipticity may range from $10^{-9}$ to $10^{-6}$ (Ushomirsky, Cutler, and Bildsten, 2000; Owen, 2006) for conventional neutron stars and somewhat larger for more exotic stars (Owen, 2005).

In order to greatly improve the sensitivity of the pulsar searches, the Einstein @ Home (2012) project distributes some of the Laser Interferometer Gravitational-wave
Observatory (LIGO) data to the home computers of an international team of volunteers. Although no gravitational waves have been detected so far, this project has detected pulsars using electromagnetic astronomical data (Knispel et al., 2011).

\section{Transients}

The signal which all ground-based detectors are aimed toward is the inspiral and merger of compact binary objects: neutron stars (NS) and black holes (BH). Perhaps 1/3 to 1/2 of the stars in the Universe have companions (Lada, 2006). Through various mechanisms, some small fraction of these can evolve into a NS/NS, NS/BH, or $\mathrm{BH} / \mathrm{BH}$ binary [white dwarfs are not quite so compact; mass transfer between the stars begins (Farmer and Phinney, 2003; Lorén-Aguilar et al., 2005) well before the inspiral signal enters the accessible band of the ground-based detectors]. These compact binaries will eventually merge after they have released their orbital energy through gravitational radiation. The Hulse-Taylor binary is one such binary; it is expected to merge in $\sim 3 \times 10^{8} \mathrm{yr}$. Estimates of the binary merger rates (Phinney, 1991; Belczynski, Kalogera, and Bulik, 2002) using bounds from astrophysical observations as well as predictions from population synthesis models vary by a few orders of magnitude. For the upcoming second generation interferometric detectors, the compact binary detection rate may be as low as $1 /$ yr or as high as 3/day (Abadie et al., 2010). A combination of extensive analytic methods (Faye et al., 2012) and high accuracy numerical simulations (Scheel et al., 2009; Szilágyi, Lindblom, and Scheel, 2009; Ajith et al., 2012) have allowed for the calculation of accurate wave forms by which one can search for these binary inspirals using matched template methods (Allen et al., 2012).

It is most likely that the largest fraction of gravitationalwave sources have not yet been modeled well enough to use a template based search. These will include sources such as stellar collapse leading to supernovae (Ott, 2009), the boiling of the cooling neutron star at the end of the collapse (Liu and Lindblom, 2001), and soft gamma-ray repeaters (Abbott et al., 2008). The most exciting prospect in making a broadband search for gravitational waves is to make a discovery of an entirely unexpected astrophysical phenomenon (Cutler and Thorne, 2002; Ando et al., 2012).

\section{Cosmic background radiation}

Starobinskii (1979) and others (Rubakov, Sazhin, and Veryaskin, 1982; Abbott and Wise, 1984) pointed out that a period of cosmic expansion in the early Universe could produce a spectrum of gravitational radiation. Allen (1988) later derived the full spectrum of gravitational waves expected from a standard inflationary universe scenario. This model predicts a nearly white spectrum (in units of energy) in the frequency band from $10^{-15}$ to $10^{10} \mathrm{~Hz}$ (Turner, 1997). This radiation from the early Universe traveled to our detectors with very little scattering along the way giving us a direct measurement of the state of the Universe at a time which is less than $10^{-30} \mathrm{~s}$ after the big bang (Weinberg, 2004). A review of prospects for detecting this inflationary background as well as possible astrophysical foregrounds is given by Allen (1997). 
There are two observational constraints on the cosmological background of gravitational waves. The relative abundances of the light elements in the Universe today tightly constrain any deviations from the standard model in big bang nucleosynthesis (BBN) (Peebles, 1993). An excess of gravitational radiation at the time of BBN changed the expansion rate of the Universe. The BBN model placed an upper limit of $\sim 10^{-5}$ (in units of the closure density of the Universe) on the energy in this primordial gravitational radiation. Certain exotic theories of the early Universe predicted higher frequency gravitational radiation (Mandic and Buonanno, 2006; Woodard, Romania, and Tsamis, 2011); for some of those models, a recent search using the LIGO detectors makes a slightly tighter bound (Abbott et al., 2009a) than from the BBN model.

\section{ALTERNATIVES TO INTERFEROMETRIC DETECTION}

\section{A. Acoustic detectors}

Attempts to make a direct detection of gravitational radiation started 50 years ago with Joseph Weber (Weber, 1960, 1970). Weber's claims of detection were never confirmed (Douglass et al., 1975; Kafka and Schnupp, 1978; Brown, Mills, and Tyson, 1982); a review of these confirmation efforts is given by Tyson and Giffard (1978).

Nevertheless, the excitement generated in the early 1970s led, in the following years, to the development of an active world-wide network of acoustic "bar" detectors with an ever increasing astrophysical reach. By the end of the 20th century, the bars had reached strain sensitivities of 3-7 $\times 10^{-19}$ for $\sim 1 \mathrm{~ms}$ bursts (Ju, Blair, and Zhao, 2000). A summary of the sensitivity of these detectors in shown in Table I.

\section{B. Pulsar timing}

In the late 1970s, Sazhin (1978) and Detweiler (1979) pointed out that the regular pulse periods of radio pulsars could be used to search for gravitational radiation in the $10-100 \mathrm{nHz}$ band. For the past three decades, astronomers have used the ever improving timing available for radio antennas and the ever increasing number of known pulsars (Hellings and Downs, 1983; Lorimer, 2008; Anholm et al., 2009; Van Haasteren et al., 2009) to search for a stochastic GW background of cosmological origin as well as the mergers of massive black holes.

TABLE I. Best sensitivity of acoustic bar detectors (Ju, Blair, and Zhao, 2000). Sensitivity is characterized by minimal detectable strain in the bar bandwidth (peak strain) and also the strain noise spectral density at the frequency of best sensitivity.

\begin{tabular}{lcccc}
\hline \hline Detector & Location & $\begin{array}{c}\text { Frequency } \\
(\mathrm{Hz})\end{array}$ & $\begin{array}{c}\text { Peak strain } \\
\left(h_{c}\right)\end{array}$ & $\begin{array}{c}\text { Strain noise } \\
{[h(f)]}\end{array}$ \\
\hline ALLEGRO & LSU & 900 & $7 \times 10^{-19}$ & $7 \times 10^{-19}$ \\
EXPLORER & CERN & 900 & $7 \times 10^{-19}$ & $7 \times 10^{-19}$ \\
NIOBE & UWA & 700 & $5 \times 10^{-19}$ & $7 \times 10^{-19}$ \\
NAUTILUS & Frascati & 900 & $6 \times 10^{-19}$ & $7 \times 10^{-19}$ \\
AURIGA & Legnaro & 900 & $3 \times 10^{-19}$ & $7 \times 10^{-19}$ \\
\hline \hline
\end{tabular}

\section{Artificial satellite timing}

Doppler tracking of man-made spacecraft was proposed as a means of detecting low-frequency gravitational waves in 1975 (Estabrook and Wahlquist, 1975). A carrier signal is sent to the spacecraft from the Earth, a transponder on the spacecraft sends the signal back, and the frequencies of the incoming and outgoing signals are compared. The relative fractional frequency fluctuations $y_{2}$ due to GWs can be written as (Wahlquist, 1987; Armstrong et al., 2003)

$$
\begin{aligned}
y_{2}[t]= & -\frac{1-\mu}{2} \bar{\Psi}[t]-\mu \bar{\Psi}\left[t-\frac{1+\mu}{2} T_{2}\right] \\
& +\frac{1+\mu}{2} \bar{\Psi}\left[t-T_{2}\right],
\end{aligned}
$$

where $\mu$ is the projection of the gravitational-wave unit wave vector onto the Earth-satellite unit vector, and $\bar{\Psi}$ is a function encoding the response of the satellite signal's response to the two polarizations of gravitational waves. The best sensitivity using this method was achieved (Armstrong et al., 2003) using the 2001-2002 data tracking the Cassini satellite. The strain noise in the $0.01-10 \mathrm{mHz}$ band ranged from $10^{-13}$ to $10^{-12} / \sqrt{\mathrm{Hz}}$. Prospects for improving this sensitivity have been explored (Armstrong, 2006); improved frequency standards, subtraction of plasma dispersion, and reduction of mechanical vibration in the terrestrial antenna may lead to as much as an order of magnitude improvement. Until a dedicated laser interferometer mission can be launched, satellite tracking will remain the most sensitive probe of gravitational waves in this frequency band (Asmar et al., 2005).

\section{Polarization of the microwave background}

At the largest spatial scales (time scales of the order of the age of the Universe), gravitational waves can be observed by measuring the polarization of the cosmic background radiation itself (Caldwell, Kamionkowski, and Wadley, 1998). The largest polarization signals are produced by the cosmological density fluctuations and would seem to swamp the small signals expected by gravitational waves. Hope is not lost, however. The gravitational waves produce a polarization vector field with a curl, whereas the scalar density perturbations do not (Hu and White, 1997). Finding this signal provided an unambiguous signal of cosmic inflation. There are many sources of foreground (Fraisse et al., 2009) contamination which must be removed in order to extract the gravitational-wave signal. These removal techniques are being actively developed by the teams pursuing polarization signals in the Wilkinson Microwave Anisotropy Probe (WMAP) (Bennett et al., 2012) and Planck (Ade et al., 2013) data as well as the numerous ground-based experiments which have been specifically designed to hunt for the polarization signal. Space-based mission concepts such as CMBpol (Baumann et al., 2009) and PRISM (Andre et al., 2013) are the ultimate word in the detection of these gravitational waves. 


\section{FUNDAMENTALS OF INTERFEROMETRIC DETECTORS}

All of the large GW laser interferometers in the past, as well as those planned for the next decade, are essentially Michelson interferometers [as opposed to, e.g., Sagnac interferometers (Sun et al., 1996)]. As Eq. (4) shows, the measured optical phase shift is proportional to the Michelson arm length; with typical parameters $(L \sim 1 \mathrm{~km}, \lambda \sim 1 \mu \mathrm{m}$, $h \sim 10^{-21}$ ) the phase shift is just $10^{-11} \mathrm{rad}$. In order to amplify the signal to detectable levels, one wants to increase $L$ by a few orders of magnitude. Unfortunately, the interferometer arm lengths are limited to a few kilometers due to practical constraints (chiefly available land and prohibitively high construction costs). In order to improve the signal-tonoise ratio, the Michelson is enhanced using several compound optical resonators.

\section{A. Delay lines versus Fabry-Pérot resonator}

In order to artificially increase the Michelson arm length, one can bounce the light back and forth in the arms to increase the interaction time with the gravitational wave, thereby increasing the optical phase shift. With sufficiently large mirrors, one could construct a Herriott delay line (Herriott and Schulte, 1965; Shoemaker et al., 1988; Beyersdorf, Byer, and Fejer, 2000) with hundreds of bounces. Drever (1983a, 1991) proposed to instead use Fabry-Pérot optical resonators in place of the delay lines. These cavities have the advantage of combining all of the many "bounces" of the delay line onto a single spot. This greatly reduces the size, and thereby, the cost, of the mirrors. An added complexity is that the Fabry-Pérot cavity must be servo controlled to be within a small fraction of its resonance linewidth in order to operate linearly.

Nearly all of the modern interferometers now use FabryPérot cavities instead of delay lines due to issues with scattered light in the latter (Schnupp et al., 1985). The technical

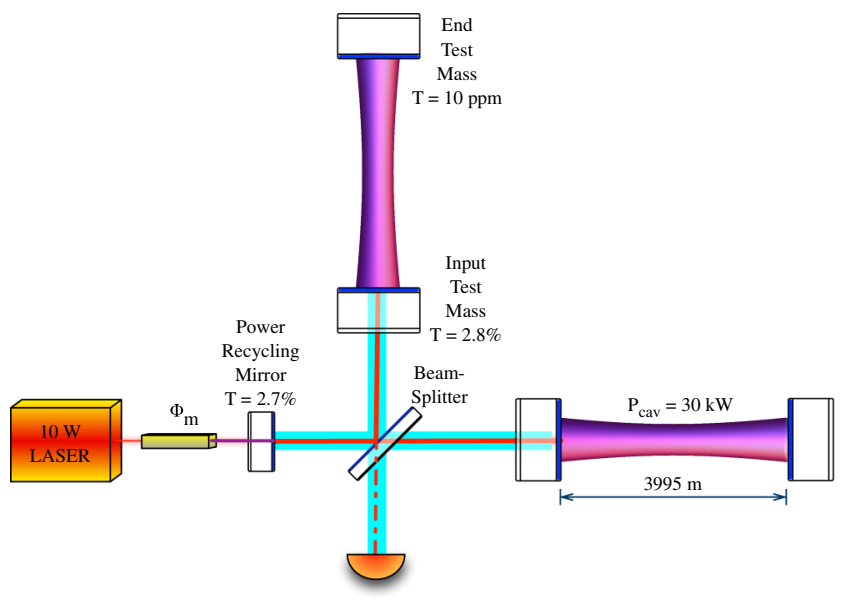

FIG. 3 (color online). Schematic of the initial LIGO interferometers. The input beam is phase modulated and then built up resonantly in the power recycling cavity. The phase modulation sidebands resonate only in the power recycling cavity. The light incident on the photodetector at the bottom of the diagram carries the GW signal. servo control issues have been largely solved over the past few decades using multidegree-of-freedom extensions (Fritschel et al., 2001; Arai, 2002; Grote, 2003; Acernese et al., 2006a) of the Pound-Drever-Hall rf heterodyne cavity locking technique (Drever et al., 1983)

Fluctuations in the alignment (Morrison et al., 1994a, 1994b; Mavalvala et al., 1998; Grote et al., 2002; Acernese et al., 2010a) and transverse beam size (Mueller et al., 2000) are sensed in a similar fashion.

\section{B. Power recycling}

The interferometer arm cavities are adjusted in length microscopically such that the fields from each arm interfere destructively at the Michelson antisymmetric port. This causes almost all of the laser light to return toward the laser. By placing a partially transmitting mirror between the laser and the Michelson beam splitter, this return light can be made to return toward the beam splitter interfering constructively with the incoming laser light. The finite transmissivity of this so-called "power recycling mirror" (Drever, 1983b) is chosen to nearly equal the total scattering losses from the Michelson's optics and thereby provide optimum power coupling from the laser source into the interferometer arms (GW transducer). In this sense, one can think of the power recycling mirror providing an impedance match to the rest of the interferometer (Fritschel, Shoemaker, and Weiss, 1992). The modern GW interferometers with Fabry-Pérot arm cavities have been able to increase the laser power impinging on the beam splitter by a factor of $\sim 65$ by using this method (cf. Fig. 3). The GEO600 detector has achieved a power gain of 1000 using power recycling (Blair et al., 2012).

\section{Signal recycling and extraction}

Just as a mirror on the symmetric side of the beam splitter can coherently amplify the power stored in the interferometer, a carefully placed mirror at the antisymmetric side of the beam splitter can amplify differential signals (see, e.g., Fig. 11). This technique is called signal recycling (Meers, 1988; Mizuno, 1995) and can be used to resonantly build up the GW signal. The GEO600 interferometer (Grote, 2010) has been successfully using signal recycling for the past several years.

The alternative strategy (which is often used in practice) is to use a kind of "antirecycling." To reduce the thermal loading due to bulk absorption in the input test masses, the Fabry-Pérot arm cavities are made to have a very high finesse. For the same arm cavity power, this lowers the power level in the optics of the power recycling cavity and allows for a high power to be stored in the cavity with minimal thermal distortions. This narrow linewidth cavity would then normally amplify only the low-frequency GW signals (and not the signals above the arm cavity pole frequency) and thereby seems like a nonsensical design choice. However, by adjusting the microscopic position of the "signal recycling mirror" to form a resonant cavity with the input test mass mirrors, the effective linewidth of the combined system (the differential arm cavity mode + the signal recycling cavity) is broadened. The signal recycling mirror's function has been transformed 
from signal recycling to resonantly extracting the GW sidebands. This technique is referred to as resonant sideband extraction (RSE) (Mizuno et al., 1993; Strain et al., 2003).

These two configurations, signal recycling and resonant sideband extraction, are the extrema of a continuous space of detuning for the signal recycling cavity. The microscopic tuning of this cavity allows for great flexibility in shaping the detector's frequency response (cf. Fig. 12).

\section{SENSITIVITY LIMITS OF LASER INTERFEROMETERS}

Laser interferometers are limited by two broad classes of noise: displacement noise and phase noise.

Displacement (or force) noises work by directly moving the interferometer mirrors. Most of these forces are filtered by the mechanical response of the mirror and its suspension and so are strongly attenuated above several hertz. Many of these force noises can be mitigated by increasing the mass of the mirror.

Phase noises produce fluctuations in the phase of the optical field used to read out the GW strain. These noise sources are modified only by the optomechanical response of the interferometer (in nearly the same way as the gravitational-wave strain) and therefore have no strong frequency dependence.

\section{A. Phase noise}

\section{Quantum vacuum fluctuations}

A "fundamental" limit to the sensing of optical phase shifts comes from the stochastic fluctuations in the arrival times of photons at the photodetector. Before 1980, the picture was that a laser interferometer could, at best, be limited by the Poisson statistics. In this picture the signalto-noise ratio (SNR) for optical sensing varied as $1 / \sqrt{P}$ (where $P$ is the input laser power) and the fluctuating radiation pressure on the mirror varied as $\sqrt{P}$. This description is similar to that of the "Heisenberg microscope" used to describe uncertainty in introductory physics courses (Feynman, 1965).

A more precise characterization of the quantum measurement limits was derived by Caves (Caves, 1981; Caves and Schumaker, 1985; Schumaker and Caves, 1985) and others (Loudon, 1981) in the early 1980s. In this picture, the noise arises from the beat between the fluctuations of the vacuum ground state of the electromagnetic field and the stable laser light: vacuum fields entering from the antisymmetric port split at the beam splitter, producing differential forces on the arm cavity mirrors (for in-phase fluctuations) and phase fluctuations (for fields that are in the quadrature phase). Vacuum fields at frequencies far from the laser frequency are rejected by the arm cavities, return to the photodetector, and beat with the static field present at the antisymmetric port (due to both, intentional and unintentional, asymmetries in the arms).

Increasing the laser power leads to a reduction in the measurement uncertainty for the mirror position but increases the amount of momentum perturbations. These momentum perturbations produce position fluctuations after a finite amount of time. Similarly, reducing the laser power reduces the momentum noise but also decreases the positional precision. For a given set of parameters, the laser power may be optimized to give the optimum strain sensitivity at a particular frequency. A detailed analysis of this quantum limit for a free mass leads to the so-called "standard quantum limit" (SQL) (Braginsky and Khalili, 1999):

$$
S_{x}(f)=\frac{2 \hbar}{m(2 \pi f)^{2}} .
$$

The SQL represents the envelope of minima in the strain noise as the laser power is tuned assuming that the amplitude and phase fluctuations from the vacuum fields are uncorrelated.

At the turn of the century, our understanding of quantum noise in interferometers was revolutionized by the work of Buonanno and Chen (2001, 2002) and Kimble et al. (2001). They showed that the combination of high power and a signal recycling cavity can build up significant quantum correlations within the interferometer. The correlation of the vacuum fluctuations can then allow for significant backaction evasion in limited frequency ranges: microscopic detuning of the signal recycling cavity leads to a radiation pressure driven restoring force. This "optical spring" can be tuned (Miyakawa et al., 2006) via the cavity detuning to optimize the response to different astrophysical sources. Further development of these quantum nondemolition (QND) techniques with application to third generation detectors is presented in Sec. VIII.A.

\section{Scattering from residual gas}

Fluctuations in the column density of gas in the interferometer arms produce noise in the measured optical phase (Zucker and Whitcomb, 1996; Takahashi et al., 2002). For a single species of molecule, the power spectral density of apparent strain fluctuations is

$$
S_{h}(f)=\frac{(4 \pi \alpha)^{2} \rho}{v_{0} L^{2}} \int_{0}^{L} \frac{\exp \left[-2 \pi f \omega(z) / v_{0}\right]}{\omega(z)} d z,
$$

where $\alpha$ is the polarizability, $\omega$ is the beam radius, $L$ is the interferometer arm length, $\rho$ is the number density, and $v_{0}$ is the most probable speed for the particle. Taking $\mathrm{H}_{2}$ as an example, it is only necessary to reach a residual pressure of $\sim 10^{-9}$ torr to reduce the induced strain noise from this molecule to $\sim 10^{-25} / \sqrt{\mathrm{Hz}}$. The partial pressure required for highly polarizable substances, such as hydrocarbons and water, is much more stringent.

\section{Backscatter}

Imperfections in the mirror shape at spatial scales larger than $\sim 1 \mathrm{~mm}$ (called "figure error;" cf. Fig. 10) can scatter the light incident on the mirrors into small angles that deposit the light into the long beam tubes. Imperfections at smaller spatial scales (called "microroughness") will produce a diffuse scatter of the light directly into the nearby vacuum chambers. A small fraction of these scattered-light fields is scattered back to the mirror and can then recombine with the circulating field via the mirror imperfections (Thorne, 1989; Winkler et al., 1994; Flanagan and Thorne, 1995; Vinet, Brisson, and Braccini, 1996). 
Seismically driven motions of the vacuum system can in this way produce phase and amplitude fluctuations of the light field within the interferometer (Schilling et al., 1981; Ottaway, Fritschel, and Waldman, 2012). Work prior to 2012 included only the terms leading to phase modulation of the interferometer's stored field. Since the relative phase between the scatterers and the interferometer field is random, there should be an equal contribution to both the phase and amplitude quadratures. With the increasingly high-power levels in modern interferometers, the amplitude component turns out to be dominant at low frequencies via the influence of radiation pressure on the mirror motion. This mechanism is analogous to that of the quantum noise in that the amplitude noise becomes dominant at low frequencies.

Careful engineering of dark, polished, scattered-light beam traps throughout the long vacuum tubes and in the vicinity of the mirrors are expected to suppress the influence of the scattered light to below the current quantum backaction limits. Backscatter from the photodetectors used for signal detection can be mitigated by moving the detectors onto a quiet, in-vacuum platform and/or using external phase modulators (Lück et al., 2008).

\section{B. Displacement noise}

All of the following effects produce motion of the test mass through stochastic fluctuation of forces. As such, the powers from these types of noise are concentrated at lower frequencies and are not important for higher frequency astrophysical sources (e.g., supernovae, millisecond pulsars, binary neutron star mergers, and ringdowns).

\section{Mirror thermal noise}

When considering fundamental sources of displacement noise for macroscopic objects (such as the interferometer mirrors) we are reminded that the equipartition theorem demands that there be $k_{B} T$ of energy per mode in any solid that is in thermal equilibrium. In order to determine what the apparent displacement noise fluctuations are for the mirrors, we computed the spectral density of fluctuations from each mode and then summed them up while including weighting factors for the effective mass in each mode as well as the spatial overlap between the mechanical eigenmode and the laser field distribution (Gillespie and Raab, 1995). This method is quite complicated and converges slowly with increasing mode number.

An alternative approach (González and Saulson, 1994; Levin, 1998) is to directly apply Callen's fluctuationdissipation theorem (Callen and Welton, 1951; Bernard and Callen, 1959; Kubo, 1966) to the mirror for the laser beam shape in question. Here the power spectrum of apparent displacement fluctuations is

$$
S_{x}(f)=\frac{k_{B} T}{\pi^{2} f^{2}}|\operatorname{Re}[Y(f)]|
$$

where $T$ is the temperature of the mirror, and $Y(f) \equiv$ $\dot{x}(f) / F(f)$ is the complex mechanical admittance (inverse of impedance) associated with the optical readout beam profile. The meaning of this is the following: in order to determine the level of apparent rms mirror fluctuation due

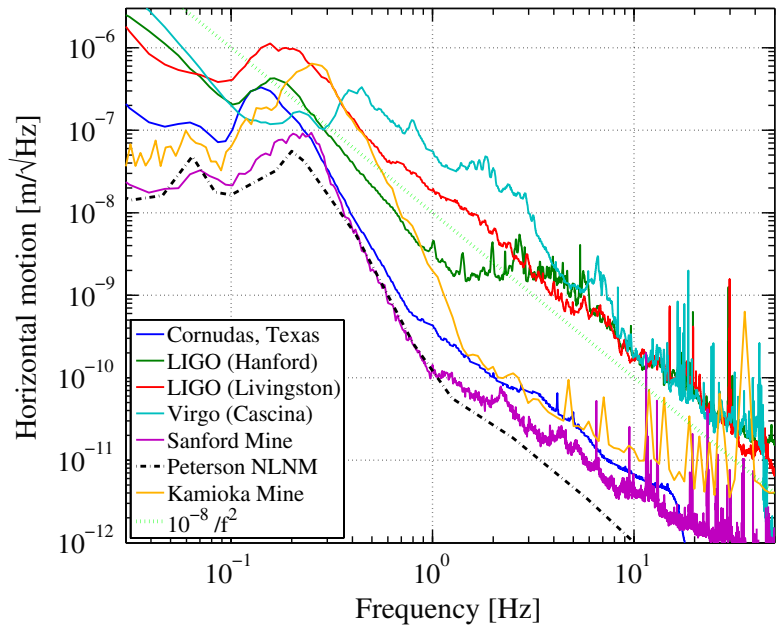

FIG. 4 (color online). The seismic vibration spectral densities shown for some of the relatively quiet sites of the current GW detector network. Also shown are two promising locations for future low-frequency detectors in the U.S.: the $4100 \mathrm{ft}$ level of the Sanford Underground Lab and a surface site near El Paso, TX. The USGS New Low Noise Model (Peterson, 1993) is included as a reference. All the spectra here [with the exception of Kamioka (Aso and Araya, 2012)] are estimated using Welch's method but with median instead of mean averaging so as to better reject non-Gaussian transients.

to thermal forces, we need only to apply a sinusoidal driving force $F(f)$ and then "measure" the response. In the case that there is no dissipation, the mechanical response of the system will be entirely in phase (modulo $180^{\circ}$ ) with the applied force. As with a classical electronic circuit, this orthogonal phase response is proportional to the dissipation: the phase shift between excitation and response is the loss angle $(\phi)$ or, equivalently, $1 / Q$ (the quality factor) of the material.

Following Levin's approach for the mirror thermal noise we can express the displacement noise power spectrum as

$$
S_{x}(f)=\frac{2 k_{B} T}{\pi^{3 / 2} f} \frac{1-\sigma}{\omega E} \phi_{\text {sub }}
$$

where $\omega$ is the spot size (1/ $e^{2}$ radius) of the beam, $E$ is the Young's modulus of the mirror substrate, $\sigma$ is the scalar Poisson's ratio for the substrate, and $\phi_{\text {sub }}$ is the loss angle. The best samples of fused silica, sapphire, and silicon can have loss angles as low as $10^{-8}$ or better and, as such, do not limit the sensitivity of modern detectors (cf. Fig. 13).

Mirror coating thermal noise.-In fact, the dominant source of thermal noise of the mirror surface is the mechanical dissipation in the dielectric, thin-film coating on the mirror surface and not the bulk mirror material. These coatings, which have very good optical qualities, are quite poor from the internal friction standpoint. The dependence of this loss on the type of material, number of layers, and layer structure has been studied extensively (Harry et al., 2002, 2006, 2007; Braginsky and Vyatchanin, 2003; Penn et al., 2003; Rowan, Hough, and Crooks, 2005; Flaminio et al., 2010; Kondratiev, Gurkovsky, and Gorodetsky, 2011; Evans et al., 2012; Hong et al., 2012; Bassiri et al., 2013).

In addition to the Brownian noise, the thermodynamic temperature fluctuations in the coating also produce noise 
(Braginsky and Vyatchanin, 2003). In this case, however, there is an additional complication: the temperature fluctuations also give rise to fluctuations in the index of refraction of the dielectric thin films. Since this thermorefractive noise has the same source as the thermoelastic noise, they must add (or subtract) coherently (Evans et al., 2008). A judicious choice of the coating layer structure can be used to mostly cancel the effects from these fundamental temperature fluctuations (Harry, Bodiya, and DeSalvo, 2012).

\section{Suspension thermal noise}

A simple example of the power of the fluctuationdissipation theorem for calculating thermal noise is the damped harmonic oscillator (Saulson, 1990). In this case, the admittance is simply

$$
Y(f)=\frac{i}{2 \pi m} \frac{f}{f_{0}^{2}+i f_{0} f / Q-f^{2}},
$$

where $f_{0}$ is the resonance frequency of the oscillator. Equation (13) gives the admittance, and thereby the thermal noise, for an oscillator damped in a viscous manner. In the absence of technical limits such as damping from gas in the vicinity of the oscillator (which can be removed through standard vacuum techniques) or friction at the top clamp (Kovalik and Saulson, 1993; Cagnoli et al., 1999) of a pendulum, the mechanical losses in low loss springs and flexures can often be characterized by a constant complex term in the spring constant $k=k_{0}(1+i \phi)$, where $\phi$, the loss angle, is also equal to $1 / Q$. This case is often referred to as structural damping.

The suspension for the mirrors of the interferometer must serve several purposes: isolate the mirror from ground vibrations, decouple the mirror from the ground to allow it to move freely in response to the gravitational waves, and hold the mirror without introducing extra thermal noise. These needs are simultaneously met by suspending the mirror as a pendulum from a thin fiber.

In contrast to a standard mechanical spring, nearly all of the potential energy for the pendulum is stored in the gravitational field (Logan, Hough, and Robertson, 1993; González and Saulson, 1994); the pendulum's gravitational spring constant is given by the simple relation $k_{g}=m g / l$, where $m$ is the mirror mass, $g$ is the acceleration due to gravity, and $l$ is the pendulum length. With this simple model, there would be no damping and the pendulum would have an infinite $Q$. In reality, there is some energy stored in the bending of the pendulum wire at the two ends. The spring constant for a pendulum supported by $N$ wires is $k_{\text {wire }}=N \sqrt{T E I} / 2 l^{2}$ (Saulson, 1990; González, 2000), where $T$ is the tension, $E$ is the Young's modulus of the material, and $I$ is the moment of the inertia wire's cross section. As this spring is much weaker than the gravitational spring, the overall loss angle of the pendulum is given by $\phi_{\text {pend }}=\phi_{\text {wire }}\left(k_{\text {wire }} / k_{g}\right)$. This reduction factor [the so-called "dissipation dilution" factor (Cagnoli et al., 2000)] is what allows for having such a low level of thermal noise in a pendulum.

\section{Seismic vibrations}

Seismic vibrations of the laboratory prove to be a lowfrequency limit for all terrestrial laser interferometers. The largest strains of the Earth's surface over kilometer scales are due to the tidal gravity from the Moon and the Sun (Melchior, 1983). These Earth tides produce length changes of 100-200 $\mu \mathrm{m}$ over a $4 \mathrm{~km}$ baseline and all of the large interferometers are compensated by long-range actuators external to the vacuum system.

In the absence of earthquakes, the next largest component of the ground motion is known as the "secondary microseism" and occurs at periods of 3-10 s (Webb, 1992). This low-frequency vibration can sometimes grow to an amplitude of several microns and must be canceled by an appropriate feedback system. Above $\sim 1 \mathrm{~Hz}$, the typical vibration spectra for reasonably quiet sites (e.g., Fig. 4) is given by (Aki and Richards, 2009)

$$
x_{G}=10^{-8}\left(\frac{1 \mathrm{~Hz}}{f}\right)^{2} \frac{\mathrm{m}}{\sqrt{\mathrm{Hz}}} .
$$

To reach astrophysically interesting strain sensitivities $\left(\sim 10^{-21} / \sqrt{\mathrm{Hz}}\right)$ with a km-scale detector therefore requires suppressing the vibrations by a factor of at least $10^{8}$ at $10 \mathrm{~Hz}$ and $10^{6}$ at $100 \mathrm{~Hz}$.

The best seismic vibration sensors reach a level of $\sim 10^{-13} \mathrm{~m} / \sqrt{\mathrm{Hz}}$ (Ringler and Hutt, 2010). Incorporating such sensors into active vibration isolation platforms (Newell et al., 1997; Hensley, Peters, and Chu, 1999) is useful in reducing the large, low-frequency motions and bringing the interferometer close to the desired operating point. The final several orders of magnitude in suppression can be achieved only by using passive isolation. In all of the laser interferometers to date, this passive isolation is roughly the same: a chain of masses and springs isolates the final test mass from the actively controlled platform.

Figure 5 shows the transfer function from horizontal motion of the ground to motion of the test mass in the laser beam

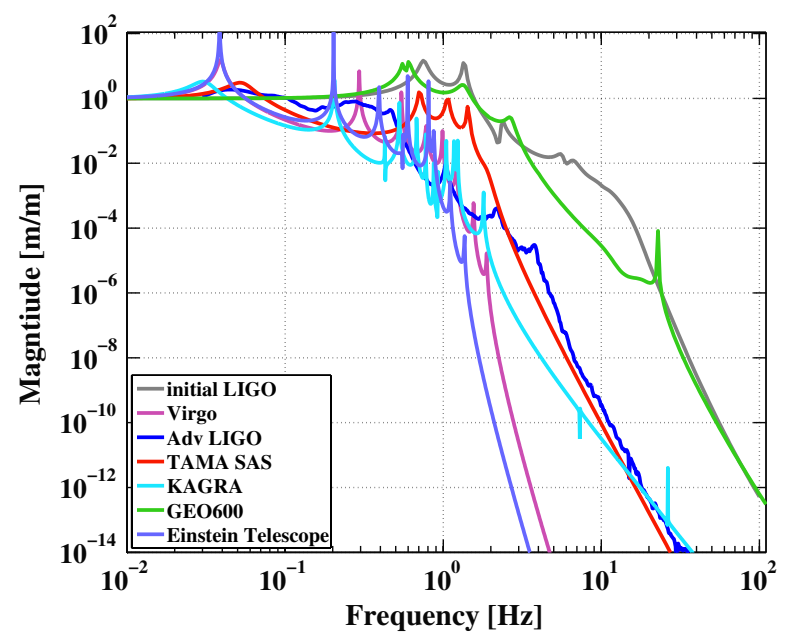

FIG. 5 (color online). Vibration isolation for the initial LIGO (Giaime et al., 1996; Ponslet and Miller, 1998), Virgo (Ballardin et al., 2001; Acernese et al., 2010b; Accadia et al., 2011b), TAMA (with SAS) (Márka et al., 2002), GEO600 (Plissi et al., 1998; Grote, 2003; Strain, 2012), Advanced LIGO (Abbott et al., 2002), KAGRA (Somiya, 2011), and the Einstein telescope cases (Einstein Telescope Science Team, 2011). In the KAGRA case, the mechanical links for cooling (included) are expected to limit the isolation performance above $\sim 1 \mathrm{~Hz}$ (Takahashi, 2012). 
TABLE II. Comparison of first generation interferometers. The numbers in the seismic row refer to the number of external active, internal passive, and pendulum suspension stages, respectively. For TAMA and LIGO, substantial hardware upgrades to the seismic isolation took place during the commissioning phase-these numbers refer to the post-upgrade configurations.

\begin{tabular}{lccccc}
\hline \hline Detector & TAMA & GEO & Virgo & LIGO 2 km & LIGO 4 km \\
\hline Arm length $(\mathrm{m})$ & 300 & 600 & 3000 & 2009 & 3995 \\
Mirror mass $(\mathrm{kg})$ & 1 & 5.5 & 21 & 10.5 & 10.5 \\
Beam spot size $(\mathrm{cm})$ & 0.85 & 2.4 & 2.1 & 3.5 & 3.5 \\
No. of seismic stages & $0+1+4$ & $1+3+3$ & $0+1+6$ & $1+4+1$ & $1+4+1$ \\
Stored power $(\mathrm{kW})$ & 1 & 5 & 20 & 30 & 50 \\
Strain noise $\left(10^{-23} / \sqrt{\mathrm{Hz}}\right)$ & 150 & 20 & 6 & 5 & 2 \\
Sensitive band $(\mathrm{kHz})$ & $0.3-10$ & $0.3-5$ & $0.02-3$ & $0.06-2$ & $0.06-2$ \\
Location & Japan & Germany & Italy & USA & USA \\
\hline \hline
\end{tabular}

direction. In most cases, this allows one to predict the motion of the test mass given the measurement of the ground noise. This assumption must be corrected for the presence of active vibration isolation systems incorporating seismometers with nonzero internal noise. The Advanced LIGO isolation system is intentionally limited by the noise of these sensors in the $1-10 \mathrm{~Hz}$ region and so, instead of the usual transfer function, the ratio of the modeled test mass motion to the ground motion is shown.

A further complication comes from the nontrivial cross couplings within the isolation systems. Vertical motion and tilts (Lantz et al., 2009; Giazotto, 2012) of the ground couple to the test mass due to mechanical cross coupling in the isolation platforms and mirror suspensions, as well as within the seismic sensors themselves. As such, the transfer functions can be considered to be only approximations of the true vibration isolation levels.

\section{Newtonian gravity noise}

Even with a much improved seismic vibration filtration system, there exists a fundamental limit to terrestrial gravitational experiments (Weiss, 1972). Density fluctuations in the atmosphere and surface waves on the ground can lead to fluctuations in the Newtonian gravitational force (also called gravity gradient noise) on the test masses. Following Saulson (1984), the equivalent strain noise is given by

$$
\delta h_{\mathrm{NN}}(f)=\frac{G}{\sqrt{3} \pi} \frac{\rho_{E}}{L} \frac{x_{\mathrm{GND}}(f)}{f^{2}},
$$

where $G$ is the gravitational constant, $\rho_{E}$ is the density of the nearby ground, $L$ is the interferometer arm length, and $x_{\mathrm{GND}}$ is the ambient ground noise. More sophisticated treatments of the correlations among the seismic waves (Beccaria et al., 1998a; Hughes and Thorne, 1998), atmospheric perturbations (Creighton, 2008), and anthropogenic influences (Thorne and Winstein, 1999) concluded that these Newtonian gravity fluctuations would nearly limit the performance of the second generation detectors in the 5-15 Hz band.

A detailed survey of the sources of vibration at the LIGO sites (Driggers and Harms, 2011) has taken into account vibrating machinery, ambient acoustics, and resonances of the surrounding structures in the laboratory; the resulting estimate is shown in Fig. 6. Although the seismic and acoustic sources that produce these forces can themselves be filtered out, there is no way to shield the test masses from their gravitational forces. It is likely that the Newtonian noise will exceed the quantum backaction limits at low frequencies (see Fig. 13). Mitigation strategies are discussed in Sec. VIII.C.

\section{Electromagnetic coupling}

In addition to the forces mentioned above, the mirrors of the interferometer may be disturbed by spurious electromagnetic forces: ambient fluctuations of the local electric and magnetic fields, as well as impacts from the background of cosmic rays.

\section{a. Cosmic rays}

At sea level, the stationary background of high-energy cosmic rays is dominated by muons. Within the typical mirror volume of $\sim 0.02 \mathrm{~m}^{2}$, there are $\sim 10-50$ muons passing through per second (Weiss, 1972; Braginsky, Ryazhskaya, and Vyatchanin, 2006). The muons deposit energy in the mirrors by exciting (or ionizing) the electrons bound by the

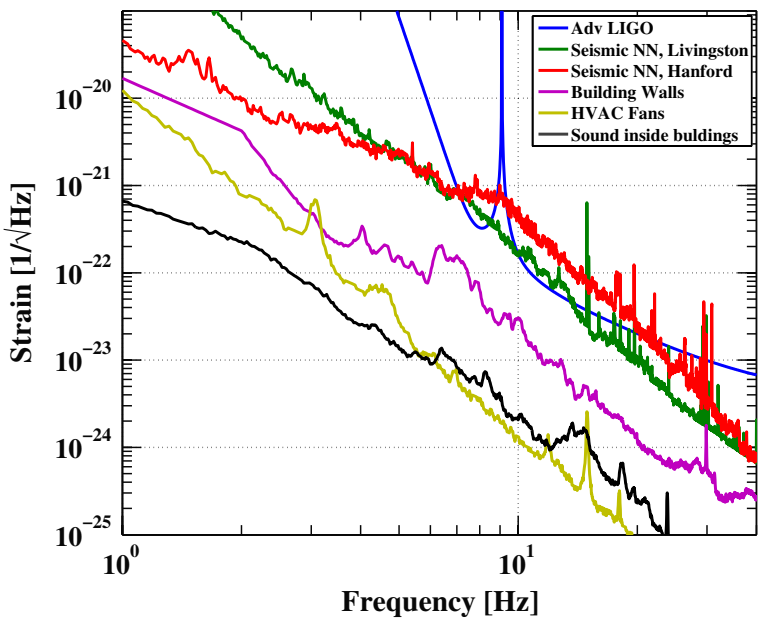

FIG. 6 (color online). Estimate of the Newtonian gravity noise at the LIGO sites compared to the fundamental thermodynamic and quantum limits for Advanced LIGO. The dominant contribution is from surface waves on the nearby ground. Vibrations of the building walls and acoustics within the building are not very significant. The turbulence from wind outside of the building primarily couples through the vibration of the building walls and is therefore already included in this estimate. 
molecules in the material. The Bethe formula (Poenaru and Greiner, 1997) tells us that most of the particles pass through the mirror depositing $\sim 100 \mathrm{MeV}$ in kinetic energy. The false alarm rate (rate of apparent GW signals) due to these highenergy muons is extremely low; they can practically be rejected by demanding a coincidence between remote interferometers for making gravitational-wave detections. The background of low energy muons, however, leads to a stationary noise spectrum given by (Yamamoto et al., 2008)

$$
h_{\text {cosmic }}(f) \simeq\left[10^{-27}-10^{-26}\right]\left(\frac{100 \mathrm{~Hz}}{f}\right) \frac{1}{\sqrt{\mathrm{Hz}}}
$$

with some variation between fused silica, sapphire, and silicon (the most common mirror materials for GW detectors). This noise source is $2-3$ orders of magnitude lower than the standard quantum limit for all of the present and envisaged future detectors.

\section{b. Ambient magnetic fields}

The ambient magnetic field fluctuation spectra are fairly broad. As measured at several sites in the U.S. they show a characteristic $1 / f$ behavior (Campbell, 1965); at the LIGO sites this $1 / f$ character has been observed with an amplitude of $B(f) \sim\left(10^{-11} / f\right) \mathrm{T} / \sqrt{\mathrm{Hz}}$. In nearly all laboratories on the Earth, the dominant features in the spectrum are the harmonics of the ac power line $(60 \mathrm{~Hz}$ in the U.S.; $50 \mathrm{~Hz}$ at the $\mathrm{GW}$ detector sites in Italy, Japan, and Germany). At lower frequencies $(5-50 \mathrm{~Hz})$, the dominant magnetic field fluctuations in the horizontal direction are due to the Schumann resonances (extremely low-frequency traveling waves within the Earth's surface-ionosphere cavity) and appear as a broad set of peaks at multiples of $\sim 7 \mathrm{~Hz}$ (Balser and Wagner, 1960; Volland, 1995). The amplitude of these peaks changes diurnally and also with the intensity of distant lightning activity.

These magnetic fluctuations couple into the interferometer chiefly through the magnets which are used to actuate the interferometer's mirrors. In the second generation GEO and LIGO detectors, the magnets have been removed from the test mass mirrors. The magnets on the next closest mirror in the suspension chain may also provide too strong of a coupling path depending upon the magnitude of local ferromagnetic components (which cause gradients).

\section{c. Surface charge}

Surface charges on the arm cavity mirrors can produce spurious forces on the mirrors through interaction with nearby conducting surfaces (e.g., the mirror suspension frames) (Mitrofanov et al., 2004; Ugolini et al., 2008; Pollack et al., 2010). These charges may build up through the friction induced by the movements of dust during the evacuation of the chambers. Random fluctuations of the charges could produce force fluctuations comparable to the thermal and quantum limits for the mirror, however, current estimates and measurements are not yet accurate enough to make the case. To be safe, several mitigation strategies are being pursued, including irradiation of the mirror surface with UV light (Sun et al., 2006) and occasionally introducing small amounts of an ultrapure ionized gas into the vacuum chambers.

\section{FIRST GENERATION DETECTORS}

The first generation of long-baseline interferometers formed the first broadband worldwide network for gravitational-wave detection. The network consisted of TAMA (300 m) near Tokyo, Japan (Takahashi et al., 2008; Arai et al., 2009); GEO (600 m) near Hannover, Germany (Grote, 2003, 2010); Virgo (3 km) near Pisa, Italy (Barsotti, 2006; Accadia et al., 2012a); and the LIGO interferometers (Abramovici et al., 1992; B. Abbott et al., 2004; Adhikari, 2004; Abbott et al., 2009c; Fricke et al., 2012)-a 4 km one in Livingston, LA and in Hanford, WA both a $2 \mathrm{~km}$ and a $4 \mathrm{~km}$ interferometer in the same vacuum system. Table II lists some of the key parameters of these detectors.

The installation and initial commissioning of these detectors started in the late 1990s. Although more advanced techniques were known at the time, these first generation instruments were built with some conservatism and therefore had several similarities. TAMA300, Virgo, and the three LIGO detectors were configured as power-recycled, FabryPérot Michelson interferometers. The GEO600 interferometer was the least conservative of all and included three "advanced" techniques: dual recycling, triple suspensions, and fused silica fibers to hold the mirrors.

The reasonably good agreement between the initial design sensitivity goals and the final performance of the LIGO and Virgo detectors (cf. Fig. 7) may lead to a false confidence in the accuracy of those early estimates. In reality, the commissioning period for all of the initial interferometers extended over several years and greatly enhanced the understanding of the large interferometers. In all cases, major hardware changes were made in order to bridge the gap between the early performance and the science goals.

In the TAMA interferometer, the initial seismic isolation was replaced with a more elaborate (Virgo-like) system to greatly reduce the seismic noise in the $1-100 \mathrm{~Hz}$ band (Takahashi et al., 2008). For the GEO600 detector, an active seismic feed-forward system, a thermal compensation system, and scattered-light mitigation techniques have been installed over the years. The Virgo interferometer was upgraded with an active thermal lens correction system, isolation optics between the laser and the interferometer

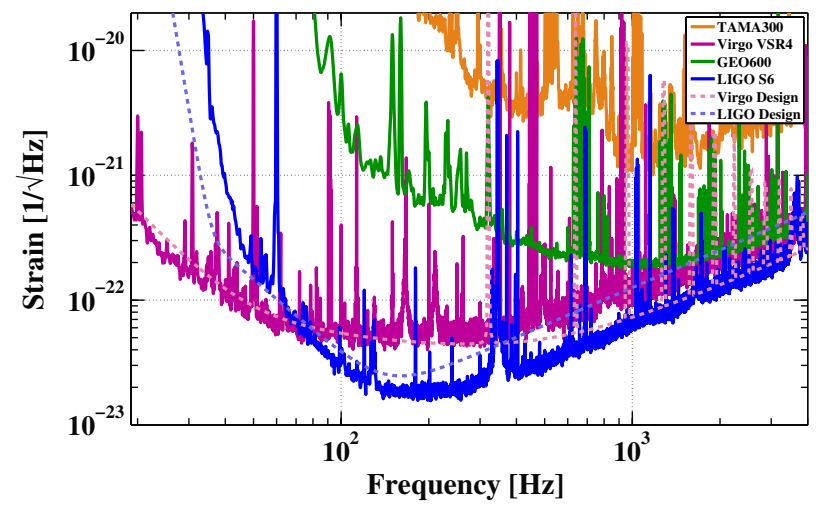

FIG. 7 (color online). Strain noise for the first generation detectors. TAMA300, GEO600, Virgo+, and Enhanced LIGO. Also shown (dashed) are the strain noise goals for the initial Virgo and LIGO detectors. 

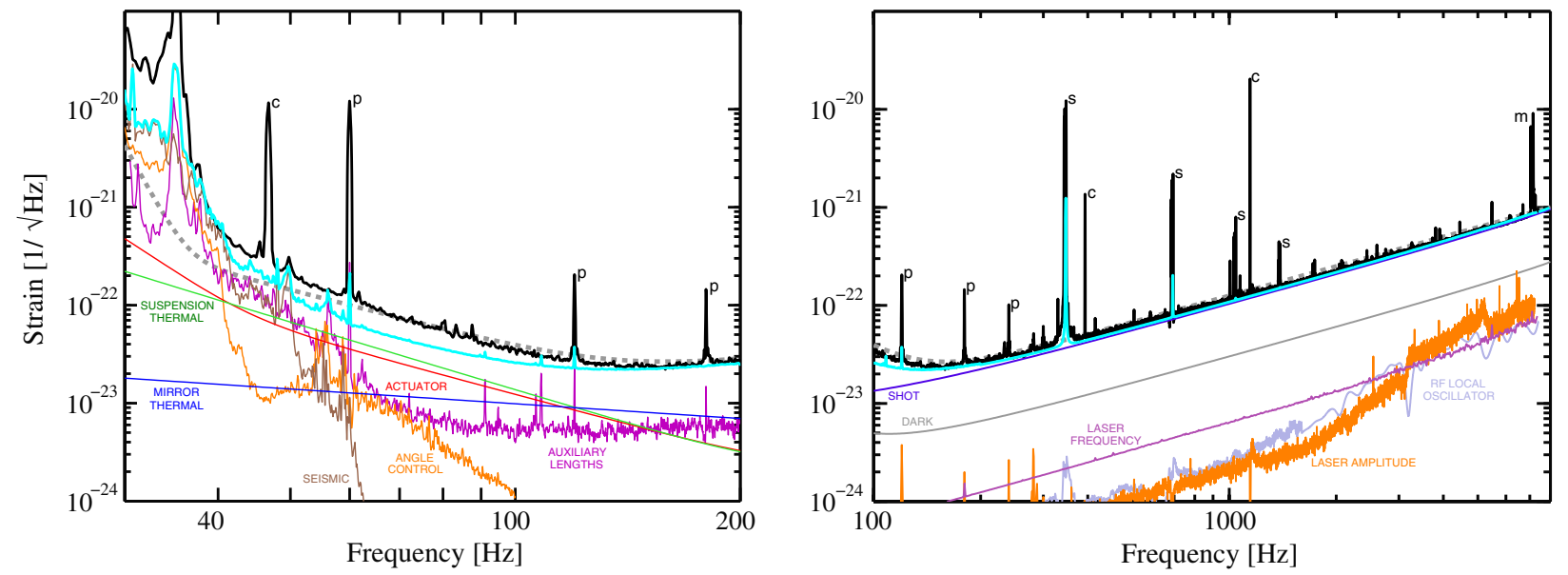

FIG. 8 (color online). Noise budget of the LIGO Hanford $4 \mathrm{~km}$ detector during the fifth LIGO science run (S5) (Abbott et al., 2009c). The left plot shows mainly the noise sources that act as a force on the mirrors. The right plot shows noise sources that appear as a phase noise on the light. The known peaks in the measured strain data are indicated as $(p)$ for power lines, $(c)$ for calibration lines, $(s)$ for the violin modes of the mirror suspension, and $(\mathrm{m})$ for the mirror's internal eigenmodes. The lighter unlabeled trace is a quadrature sum of all known noise sources and the darker unlabeled trace is the measured strain output of the interferometer. The discrepancy between these two traces remains unexplained but is suspected to be due to excess friction in the suspension wire attachments to the mirror. The dashed trace is the initial LIGO Science goal.

(Virgo Collaboration, 2008, 2010), as well as numerous control system upgrades (Accadia, Acernese et al., 2011; Accadia et al., 2011a). LIGO also added a thermal compensation system as well as an active seismic isolation system for the Louisiana interferometer (R. Abbott et al., 2004; Hardham et al., 2004), acoustic isolation chambers for the external optics, and an extensive upgrade to the digital control system.

Between the operation of the initial detectors and installation of the second generation detectors, there was an additional scientific data taking run which followed major hardware upgrades of the Virgo and LIGO detectors (Virgo+ and enhanced LIGO) which incorporated the noise analyses of the initial detectors (e.g., Fig. 8) and several technologies in development of the second generation machines.

In the remainder of this section, the most significant unexpected or nonideal features are described as well as the associated mitigation strategies.

\section{A. Excess optical loss}

With the use of power recycling, nearly all of the laser light is coupled into the interferometer. Good matching between the interferometer arms ensures that only a small fraction (of approximately a few percent) escapes out of the antisymmetric port. Most of the laser power entering the interferometer is scattered into the surrounding vacuum system. For all of the interferometers, the measured optical losses were significantly higher than expected from the initial, tabletop measurements (Sato et al., 1999). A small fraction of the losses came from absorption in the mirror substrate and on the high-reflectivity dielectric mirror coatings within the FabryPérot arms (in the case of LIGO, Virgo, and TAMA) (Hild et al., 2006; Ottaway et al., 2006; Brooks et al., 2009). Depending upon the level of contamination, the absorption of the mirror surfaces ranged from 1-10 ppm, leading to a wide range of problematic thermal gradients in the mirrors.
Scatter losses.-As described next (Sec. VII.B), perturbations in the mirror surface can scatter light out of the interferometer. This scatter loss is the chief limit to the power buildup within the resonant cavities. Although $\sim$ ppm level losses have been observed in small optical cavities (Rempe et al., 1992; Uehara et al., 1995), the round-trip losses in the Fabry-Pérot arms of these large interferometers ranged from $100 \mathrm{ppm}$ (LIGO) to $300 \mathrm{ppm}$ (Virgo) (Acernese et al., 2007). A small fraction of this was due to point defects (cf. Fig. 10) in the mirror coating. The largest fraction of the loss was due to mirror surface perturbations at the scales of several centimeters.

\section{B. Optical cross coupling}

All of these interferometers were designed with a high level of symmetry to passively reject many noise sources. Differential phase shifts in the interferometer arms (e.g., strain from a gravitational wave) directly produce a signal at the antisymmetric port. Fluctuations of the incoming laser light or motions of the other mirrors also coupled through to the GW channel in sometimes unexpected ways and new techniques were developed to combat these issues.

\section{Fluctuations of the light}

The Michelson topology, in particular, is largely insensitive to amplitude and frequency fluctuations of the illuminating laser light. By adjusting the length of the interferometer arms microscopically, the antisymmetric port is made to be nearly dark. In this "dark fringe" condition the commonmode rejection ratio for laser frequency noise was found to be 200-1000 for the various interferometers, limited by the imbalance in scatter losses between the arms. Laser power fluctuations can directly drive the mirrors through radiation pressure and an imbalance of the power in the arms. Power fluctuations can also produce apparent mirror fluctuations due 
to gain modulation of quasistatic offsets in the length control feedback loops of the interferometer. Some of these operating point fluctuations are driven by seismic motion and so this noise source comes from the product of seismic motion and laser power fluctuations.

\section{Local oscillator phase noise}

In the TAMA, LIGO, and Virgo interferometers, the scheme that was used to read out differential arm cavity strain is similar, mathematically, to the standard Pound-Drever-Hall technique which is widely used with simple, rigid cavities. An important difference, however, is that both the carrier field and the rf sidebands travel through a few optical cavities before being optically recombined on the antisymmetric port photodetector (cf. Fig. 3).

In principle, phase noise of the oscillator used to generate the rf sidebands canceled during the demodulation of the heterodyne signal. The electronic local oscillator signal used in the demodulation does not, however, experience the same temporal filtering that the optical sidebands do. Furthermore, the filtering experienced by the optical sideband fields is not as simple as might be envisaged by modeling the process by propagation of plane waves (Camp et al., 2000). Each of the higher-order transverse modes of the sideband field experiences a different phase shift in the cavities (Ballmer, 2006; Grote, 2008). In this way, the final recombined signal depends in a detailed way on the mirror surface perturbations and, as explained in Sec. VI.E, on the thermal state of the recycling cavity optics.

In order to reduce this noise to below the shot-noise limits, multiple strategies were employed: the mirror curvatures were adjusted with auxiliary heating lasers, the cavity lengths were microscopically adjusted to match the optical and electronic paths, and finally, an ultra-low-noise crystal oscillator (Wenzel, 2012) was used to reduce the source term by an order of magnitude (to a phase noise level of $<-160 \mathrm{dBc} / \mathrm{Hz})$.

\section{Motion of auxiliary mirrors}

Longitudinal motions of the other mirrors in the interferometer (e.g., the power recycling mirror and the beam splitter) weakly coupled to the GW readout (Regehr, 1995), but not so weakly that they can be completely neglected.

The motion of the beam splitter (or, more precisely, differential motion of the short Michelson interferometer formed by the beam splitter and the input test masses) couples in the usual way; the mirror motion modulates the phase of the carrier field and produces a signal as if it were a gravitational wave. This produces a weaker signal since it does not experience the resonant buildup of the arm cavities. However, this mirror has noise imposed on it by its feedback control loop which is orders of magnitude above the shot-noise limits of the GW channel. In order to recover the quantum limited performance of the interferometer, this feedback noise was filtered and injected into the end mirrors so as to cancel the initial noise injection (Fritschel et al., 2001). This feedforward path was able to cancel the noise by a factor of 30-100 (for LIGO) in the most sensitive frequency band. The Virgo feed-forward system achieved several times more cancellation by using an adaptive gain in this path (Acernese et al., 2010c).

The coupling of the power recycling mirror motion is less straightforward. This motion produces a signal only through the existence of asymmetries. The imbalance in the amplitude reflectivity of the arm cavities produces a carrier field at the antisymmetric port which is in the orthogonal phase from the gravitational-wave signal sidebands. The power recycling mirror motion modulates the phase of the rf sidebands and couples this orthogonal phase field into the GW channel. This coupling was $\sim 10$ times smaller than the Michelson coupling, but was dealt with in essentially the same way, although the achieved cancellation factor was several times smaller.

Removing these noise sources allowed the interferometers to operate much closer to their fundamental limits. An unpleasant side effect is that the residual noise from these processes is highly nonstationary, almost by definition. The static coupling path is canceled by these electronic cancellation paths, but time variation in the optomechanical properties of the interferometers (due to temperature, seismic noise, beam pointing, optical losses, etc.) produces large fluctuations in the residual coupling. The next generation interferometers have the added complexity of also needing to cancel the motion of the signal recycling cavity, but the added benefit of having much less low-frequency mirror motion resulting in less variation in the coupling constants.

\section{Low-frequency mirror motion}

Simple estimates of the coupling of seismic vibration (e.g., Sec. V.B.3) to the interferometer's strain channel assume that the coupling is essentially linear. During the decade spent commissioning these interferometers, it became clear that this assumption fails in a myriad of ways: large, lowfrequency motion produces noise in the GW detection band.

\section{Seismic amplification}

As can be seen from Fig. 5, below $1 \mathrm{~Hz}$, many of the isolation systems amplify the ground noise. In the case of the passive systems, this comes from the lowest natural frequencies of the stacks and suspensions. In the case of the active systems, this can come from the coupling of tilts into the active sensors or insufficient phase margin in the control systems. As is well known from electronic filter design, it is necessary to have some high resonances in the passband in order to have steep attenuation in the stop band for reactive, low-pass filters. Such is also the case for these mechanical vibration filters; damping the low-frequency resonances leads to performance degradation in the GW band. Unfortunately, this design tradeoff leads to an amplification of motion in the anthropogenic band which can be highly nonstationary (Acernese et al., 2004; Daw et al., 2004; Saccorotti et al., 2011; Accadia et al., 2012b).

\section{Noise from damping}

In order to mitigate this problem, the suspension systems were designed to have some capability of using "cold damping": sensors local to each test mass can be used to sense and 
suppress these high amplitude, low-frequency motions. This is only partially successful. Although it is possible to reduce the motion somewhat, it proved impossible to completely compensate the amplification without introducing excess noise into the GW signal band. The feedback filters must obey the Kramers-Kronig relations.

For the interferometer mirrors in the recycling cavity or Michelson area, the situation is more complicated. Optics that are separated by much less than a seismic wavelength move coherently. In the absence of active feedback systems, the differential motion among these optics is highly suppressed. The noise of the local damping sensors has no such correlation, however. Attempting to apply damping in such a situation actually amplifies the relative interferometric length fluctuations at low frequencies. In practice, these issues require the delicate tailoring of the local damping feedback filters and limits how strong the damping of the high $Q$ mechanical resonances can be.

\section{Nonlinear noise generation}

These large, low-frequency motions all conspire to produce noise in the GW band through several different nonlinear mechanisms.

\section{Bilinear angle to length conversion}

The large ground motions in the $0.1-1 \mathrm{~Hz}$ band produce angular fluctuations in the interferometer mirrors through cross couplings in the vibration isolation and suspension systems; the source of the angular motion is chiefly horizontal motion of the ground and not tilt. These fluctuations are partially canceled by a complicated control system (Mavalvala et al., 1998; Grote et al., 2002; Acernese et al., 2006b) based on rf heterodyne detection of the optical wave fronts on quadrant photodetectors and feedback through a MIMO (multiple input-multiple output) digital signal processing system. The control system feeds some of the sensor noise in the GW detection band back into the mirrors. The mirror actuators are balanced so as to place the axes of rotation of the mirror at the center of the laser beam spot position and this cancels the coupling of angular noise to interferometer strain readout, to first order (Tatsumi, Arai, and TAMA Collaboration, 2006). Because of the residual low-frequency mirror motions, the resonating laser beam moves around with respect to this null point by hundreds of microns. The angle to strain coupling is therefore, nonstationary (Dooley, 2011). During intense storms or times of high anthropogenic seismicity, the low-frequency noise of the detectors becomes compromised by this nonstationary noise source.

\section{Actuator nonlinearities}

In order to maintain the resonance condition of the interferometer, the control system must compensate for the approximate micron scale motions below $1 \mathrm{~Hz}$ while simultaneously introducing less than $10^{-19} \mathrm{~m}$ of motion in the GW band around $100 \mathrm{~Hz}$. This requires the mirror actuator to be highly linear: the upconversion of force noise must be less than 1 part in $10^{9}$. While such a high dynamic range is possible with just modern low noise electronics, it is not feasible to do so using magnetic actuators, due to the Barkhausen effect (Barkhausen, 1919; Bittel, 1969; Bertotti, 1998; Durin and Zapperi, 2004). The low-frequency control forces are applied to the mirror using magnet-coil pairs. The time-varying control forces, which are used to compensate for the seismic motions, induce domain flips in the more loosely bound domains of the magnets attached to the mirrors. In the $\mathrm{NdFeB}$ magnets used in LIGO and TAMA, there were many weakly bound domains and the Barkhausen effect exhibited a force noise upconversion of 1 part in $10^{7}$. The Virgo interferometer was instrumented with SmCo magnets which have a much smaller Barkhausen effect. However, any nearby ferromagnetic materials can lead to this fluctuating magnetic noise (Schofield, 2010). Future interferometers are being designed to use multiple chain pendulums (as in Virgo and GEO) so as to minimize the dynamic range requirements. To minimize the magnetic coupling, the magnets will be either downsized drastically or eliminated altogether in favor of electrostatic actuators.

\section{E. Thermal distortions}

The small, but nonzero, optical absorption in the mirrors of the interferometers produced significant thermal gradients within the optics. These gradients produced distortions of the mirror surface (thermal expansion of the glass) as well as a significant thermal lensing within the substrate (temperature dependence of the refractive index) (Hello and Vinet, 1990, 1993; Winkler et al., 1991; Strain et al., 1994). The presence of low levels of contaminants on the optics' surfaces led to higher than anticipated levels of absorption.

There are several mechanisms by which thermal distortions can lead to instability and degraded noise performance in the interferometers. The simplest mechanism is through reduction of signal; differential thermal lensing in the input test mass mirrors reduces the spatial overlap of the GW signal sidebands with each other. This contrast defect also increases the shot-noise level at the antisymmetric port. Thermal lensing in the recycling cavity optics can also destabilize the angular control system by reducing the sensitivity to certain degrees of freedom and destabilizing the feedback control matrix.

A particular optical design choice exacerbated some of these problems. The recycling cavities were made much shorter (for practical reasons) than the long arms. With the large beams required for low thermal noise, such short cavities are geometrically unstable [cavity $g$ factor (Siegman, 1986) near unity] (Gretarsson et al., 2007). Small thermal distortions were found to drive the system into instability due to the degeneracy among the higher-order spatial modes.

In order to compensate for this effect, active thermal correction systems were installed to smooth out the thermal gradients (Lück et al., 2004; Lawrence et al., 2004; Ballmer, 2006; Accadia, Swinkels, and V. Collaboration, 2010; Rocchi et $a l ., 2012)$ as well as to improve the fringe contrast at the antisymmetric port.

Because of the troubles with degenerate cavities, the KAGRA and Advanced LIGO detectors are adding extra optics in their design to break the modal degeneracy in the 
recycling cavities (Arain and Mueller, 2008; Granata, Barsuglia et al., 2010).

\section{SECOND GENERATION DETECTORS}

The purpose of the second generation interferometers is to achieve such a strain sensitivity that the detections of gravitational waves should become fairly regular, enabling the use of these detectors as astronomical tools. They are roughly an order of magnitude more sensitive than the first generation detectors (cf. Fig. 18). The worldwide network comprising Advanced LIGO (Harry, 2010; LIGO, 2011), Advanced Virgo (Virgo Collaboration, 2011, 2012), GEO-HF (Lück et al., 2010), and KAGRA (KAGRA, 2011; Somiya, 2011) all use power and signal recycling and a variant of tunable resonant sideband extraction.

\section{A. Monolithic silica suspensions}

The first generation LIGO and Virgo interferometers were somewhat limited by thermal noise in the mirror suspensions. This was partially due to the intrinsic dissipation of the steel wires and partially due to excess friction in the wire attachments (Abbott et al., 2009c). In order to avoid both of these problems, the new suspensions are nearly monolithic: instead of a steel pendulum wire, a high-quality fused silica [sapphire for KAGRA (Uchiyama et al., 1998; Tomaru et al., 2002)] fiber is drawn and then bonded directly to silica attachments on the mirror barrel (Robertson et al., 2002; Aston et al.,

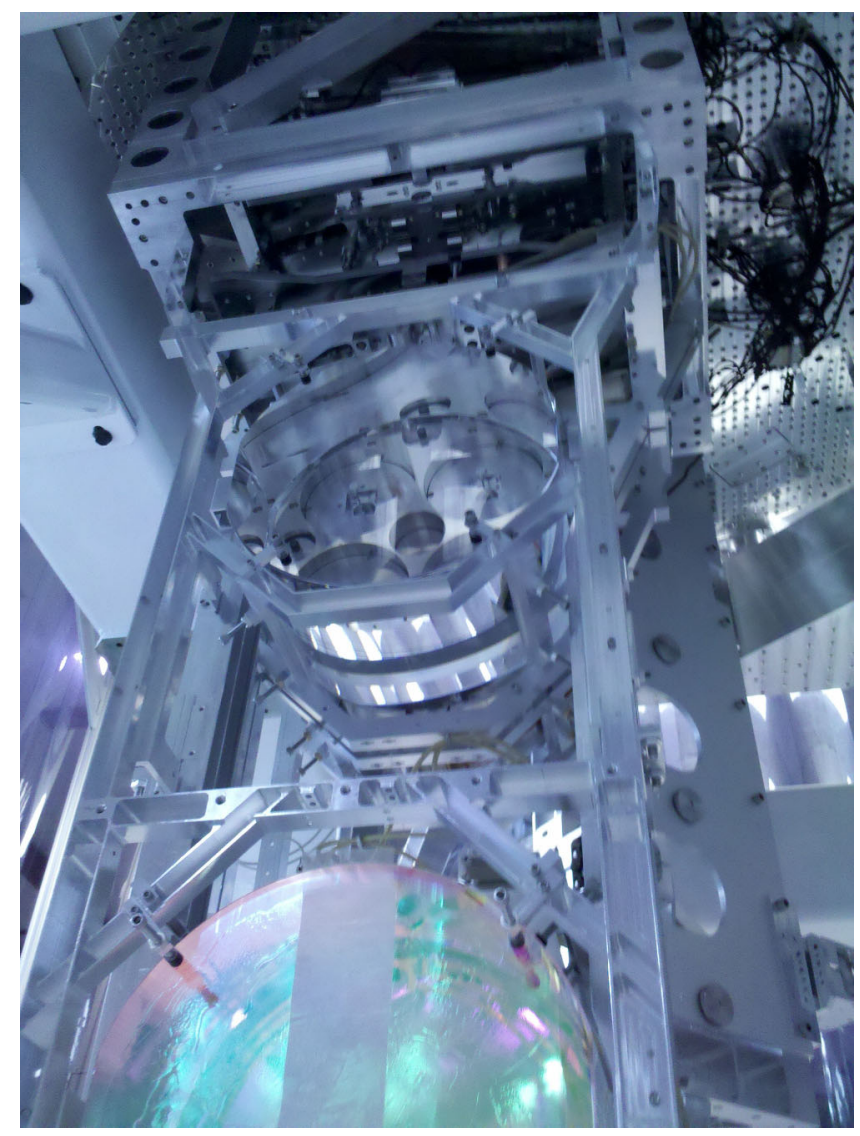

FIG. 9 (color online). Advanced LIGO quadruple suspension. The final stage is a $40 \mathrm{~kg}$ mirror suspended by four laser-welded silica fibers.
2012; Cumming et al., 2012). As most of the elastic energy (and therefore the dissipation) is concentrated near the bending points at the ends (cf. Sec. V.B.2), the cross sectional shape, near the ends, is optimized with respect to the noise (Cumming et al., 2009). This type of silica suspension has been used in the GEO600 and Virgo+ interferometers. An example Advanced LIGO suspension is shown in Fig. 9.

As with the mirror coatings (cf. Sec. V.B.1), both the dissipation due to internal friction and the thermoelastic (Zener) damping need to be considered. For highly stressed fibers, one must also consider the temperature dependence of the Young's modulus $(d Y / d T)$ : the fundamental thermodynamic temperature fluctuations which produce the usual thermoelastic noise via the thermal expansion coefficient also drive the stressed suspension fiber by changing the Young's modulus (Cagnoli and Willems, 2002). Fortuitously for LIGO and Virgo, fused silica has a positive $d Y / d T$; the result is that an appropriate fiber diameter can be chosen to cancel these thermoelastic effects.

Finally, studies of the fiber's mechanical loss as a function of fiber dimension have revealed that $Q$ is limited by defects in the fiber's surface (Gretarsson and Harry, 1999; Gretarsson et al., 2000; Penn et al., 2006; Heptonstall et al., 2010; Hammond et al., 2012) and not by the intrinsic mechanical dissipation of fused silica. Several decades of experience with surface treatment of quartz oscillators and quartz fibers have influenced the current design for GW detectors. The fibers which are now used for the Advanced LIGO mirrors are pristine with a small concentration of residual defects in the surface. These defects in addition to the losses in the welded attachment point dominate the Brownian thermal noise in the suspension. Techniques for evading this limit for the next generation are described in Sec. VIII.D.

\section{B. Mirror metrology}

To support Gaussian beam shapes in the Fabry-Pérot cavities, the mirrors are polished to have spherical profiles. Deviations from the ideal shape reduce the overall interferometer performance in a number of ways. Roughly speaking, perturbations at small spatial scales promptly scatter light out of the cavity. Larger scale defects distort the ideal $\mathrm{TEM}_{00}$ eigenmode of the arm cavities. To compute the power lost into wide angles, one needs to know only the bidirectional reflectance distribution function (Bass and Mahajan, 2009) of the mirror, which is readily obtained from measurements of the mirror surface map (Walsh, Leistner, and Oreb, 1999; Yamamoto, 2007). To first order, this distinction between small and large scales can be made in the following way: light scattered from a mirror which falls off the opposing mirror of the cavity is lost and does not contribute to the cavity mode distortion. In the LIGO case this corresponds to an angle of $\theta_{\text {lost }} \sim r_{\text {mirror }} / L_{\mathrm{FP}}$ and a spatial scale of $x_{\text {rough }} \sim$ $\lambda / \theta_{\text {lost }} \sim 2 \mathrm{~cm}$.

For the larger spatial scales the situation is complex; the scattered field is captured on the far mirror and so it is not precisely "lost." Rather, the resulting distortion in the cavity field results in an imperfect interference at the Michelson antisymmetric port. At the smaller scales, however, a good approximation for the power lost due to surface roughness is 

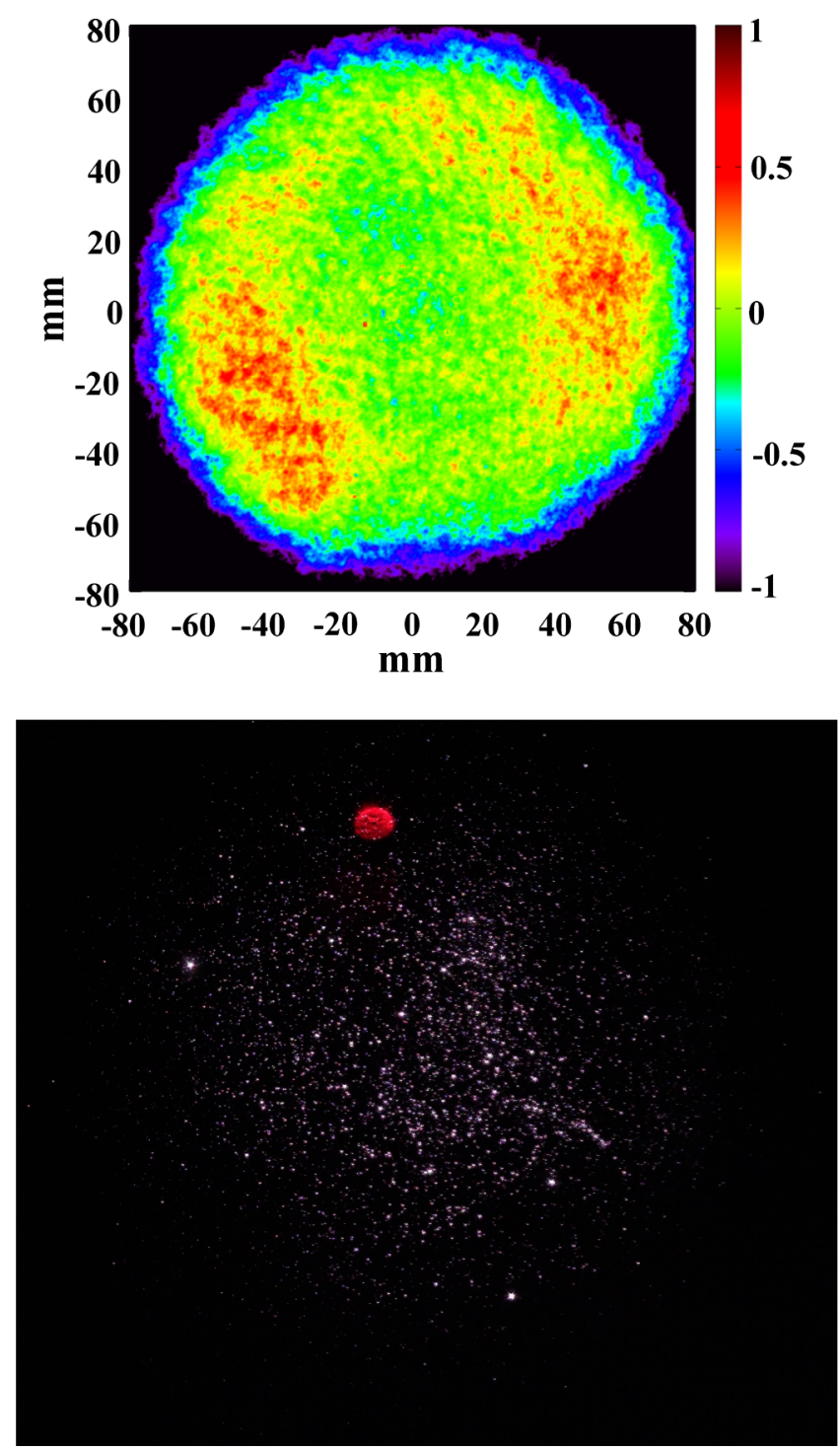

FIG. 10 (color online). (Top) Surface phase map (in units of nanometers) of one of the Advanced LIGO arm cavity mirrors after applying the high-reflectivity mirror coatings. From ZygoEPO, 2011. (Bottom) Infrared image of an initial LIGO arm cavity mirror taken with the cavity locked, highlighting the abundance of point defects. The gray oval is the diffuse scatter from an auxiliary beam used for tracking the mirror angle. From Vorvick, 2012.

$$
\frac{P_{\text {scatter }}}{P_{\text {incident }}}=\left(\frac{4 \pi \sigma}{\lambda}\right)^{2} \text {, }
$$

where $\sigma$ is the rms surface roughness and $\lambda$ is the laser wavelength. Finally, at the smallest scales the dominant source of the loss is a random distribution of point scatterers. The ultimate nature of these points has not been currently discovered; the common wisdom is that they are density or index defects in the dielectric coatings. The scatter from these points is therefore treated as either Rayleigh or Mie scattering (depending upon the defect size).

Power loss limits the maximum achievable power recycling buildup, reduces the maximum benefits achievable from QND techniques by degrading the quantum entanglement of the light (cf. Sec. VIII.A.4), and introduces technical noise from backscatter (cf. Sec. V.A.3). Over the past decade, an intense development effort has led to improvements in the mirror polish on both long and short scales. The combination of extremely accurate metrology (Sykora and de Groot, 2011) of the mirror profile and the use of ion beam figuring has resulted in an order of magnitude smoother mirror (see Fig. 10) than the first generation GW interferometers. It remains to be seen if a similarly good surface can be achieved for sapphire [as is planned for KAGRA (Uchiyama et al., 1999; Somiya, 2012)] or silicon (which is being considered for future cryogenic detectors).

\section{Dual recycling}

The dynamic tuning capability of signal recycled interferometers (e.g., Fig. 11) is a powerful one and makes these instruments qualitatively different from their predecessors. By adjusting the length of the signal recycling cavity by fractions of a wavelength, the coupled resonance between the arm cavities and the signal mirror can be fine-tuned to match the frequency content of astrophysical sources as shown in Fig. 12. The low-frequency response is due to the radiation pressure induced optical spring (cf. Sec. V.A.1) and can also be tuned by adjusting the laser power. Both the signal mirror position and the laser power can be adjusted remotely to arrive at a new configuration within minutes, in principle.

The baseline configuration of the Virgo and KAGRA interferometers will have the signal recycling cavity slightly detuned from resonance in order to maximize the sensitivity to a specific astrophysical source: the inspiral of a binary neutron star system $\left(M_{1}=M_{2}=1.4 M_{\odot}\right)$. The Advanced LIGO and GEO-HF interferometers will begin operation in a broadband resonant sideband extraction configuration.

Mode healing.-In addition to the ability to tune the response of the interferometer to the space-time strain, the interferometers with signal recycling cavities also exhibit the phenomenon known as mode healing (Meers, 1988; Strain and Meers, 1991; McClelland et al., 1993; Heinzel et al., 1998). Without this mirror, differences in the spot size or wave-front curvature of the beams from the two Michelson
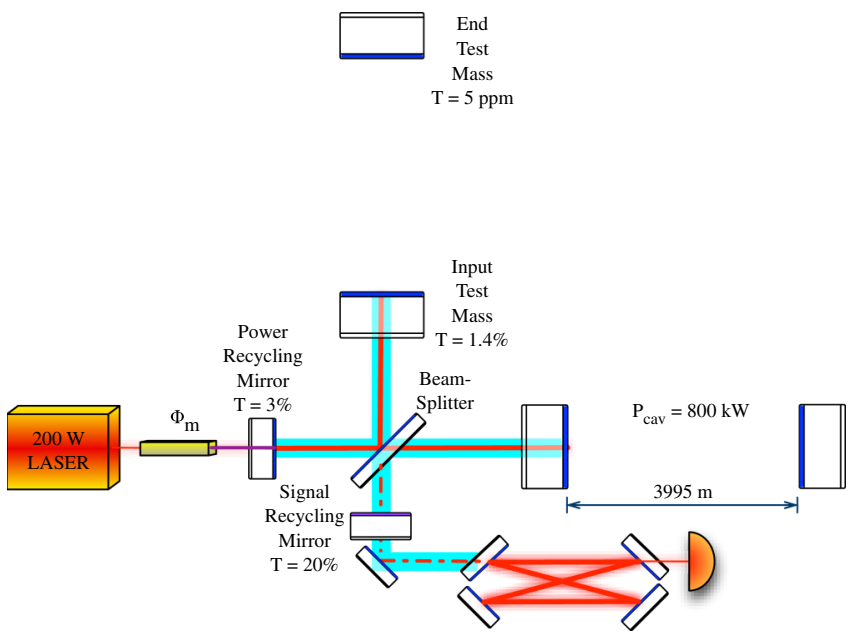

FIG. 11 (color online). Schematic of the Advanced LIGO interferometers. The output beam at the antisymmetric port is filtered by a rigid bow-tie cavity to remove the rf sidebands and the higherorder spatial modes that come from distortions in the optics. 


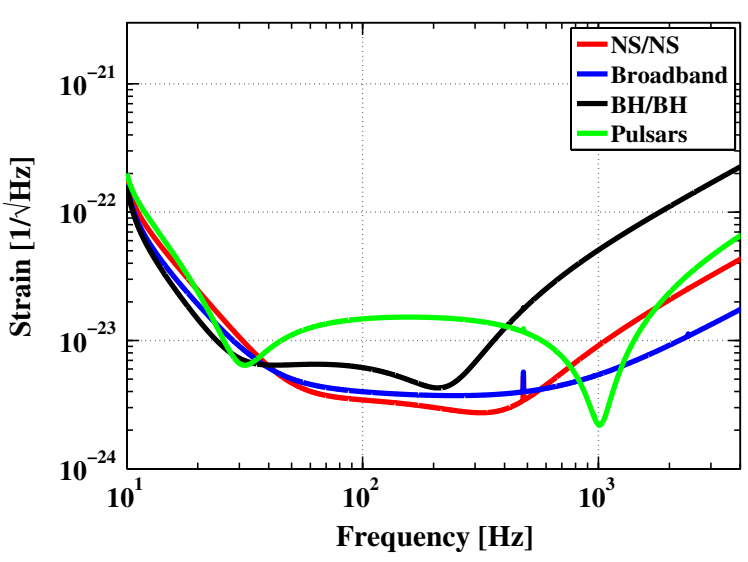

FIG. 12 (color online). Detector configurations to target particular astrophysical sources. The optimal tunings of the Advanced LIGO interferometer for (NS/NS) neutron star binary inspirals, $(\mathrm{BH} / \mathrm{BH})$ for intermediate mass black hole binary inspirals, and (pulsars) for narrow-band sources (such as pulsars) emitting gravitational radiation around $1 \mathrm{kHz}$ are shown. The broadband configuration has the best overall sensitivity and is expected to be the easiest to operate.

arms result in an imperfect destructive interference at the Michelson antisymmetric port (where the GW signal is recorded). This extra light produces no signal but contributes to extra shot noise as well as introducing technical difficulties with the interferometer control system (Smith-Lefebvre et al., 2011). The signal recycling cavity can be designed to be antiresonant for this "junk light" so as to preferentially keep it from getting to the detection port while allowing the signal light to pass (Bochner, 2003; Pan, 2006). With different storage times for each higher-order spatial mode, some of the energy which is initially scattered out from the fundamental mode can come back into this mode due to the mode mixing which occurs at each perturbed optical surface. Depending on the details of mirror roughness, signal cavity tuning, and $g$ factors of the arms and signal cavity, there can be either mode healing or mode harming for the fundamental mode.

\section{High-power optomechanics}

These new high-quality optics make it possible to use massively higher power levels. The designs of the advanced interferometers call for storing 0.5-1 MW in the arm cavities in order to improve the shot-noise limited sensitivity (shown in Fig. 13); this is a factor of $10-50$ higher than the previous generation.
While some differences exist among the laser designs for LIGO, Virgo (Gréverie et al., 2010), and KAGRA, they share a set of common themes. First, a low noise master oscillator $(\sim 1-2 \mathrm{~W})$ is amplified with one or two amplifier stages. The light is then passed through a low finesse cavity in order to filter angular fluctuations and to provide filtering of amplitude noise at rf frequencies.

The LIGO design has a $2 \mathrm{~W}$ Innolight nonplanar ring oscillator as the master oscillator, followed by a single pass power amplifier with an output of $35 \mathrm{~W}$. This configuration was used as the laser for the Enhanced LIGO (Fricke et al., 2012). This $35 \mathrm{~W}$ system has now been augmented by a highpower stage to produce $200 \mathrm{~W}$ of single mode light at $1064 \mathrm{~nm}$ (Willke et al., 2008).

With the direct (homodyne) readout scheme adopted for LIGO, Virgo, GEO, and KAGRA, the laser power fluctuations show up directly in the readout signal. At high-power levels, the dominant coupling path for laser power fluctuations is not so direct. The classical radiation pressure from the laser power fluctuations pushes the mirrors directly and couples to the antisymmetric port through the imbalance in the finesse of the arm cavities. To mitigate this somewhat, multistage active stabilization is used to suppress the raw laser noise by several orders of magnitude. In the end, the relative power stability of the light (shown in Fig. 14) entering the interferometer is $\lesssim 10^{-8} / \sqrt{\mathrm{Hz}}$ in the GW band (Kwee, Willke, and Danzmann, 2009).

The sensitivity to laser frequency noise is expected to be no greater than it was for the first generation detectors. Therefore, the same strategy of using a multistage active stabilization scheme (Fritschel et al., 2001; Abbott et al., 2009c; Acernese et al., 2009) is expected to be sufficient.

\section{Angular instabilities}

In 2002, the LIGO interferometers were beset by weak angular instabilities as the stored powers in the arm cavities exceeded $\sim 1 \mathrm{~kW}$. Sidles and Sigg (2006) pointed out that these instabilities must be due to radiation pressure overwhelming the mechanical restoring torques of the mirror suspensions.

The mechanism behind this "Sigg-Sidles" instability is illustrated in Fig. 15. In this picture the radiation pressure couples the suspended optics at either end of the cavity. Including this optical torque, the two mirror system can now be seen as having a "soft" and "stiff" mode. With enough stored optical power, the radiation pressure torque can statically destabilize the cavity in the soft mode.

TABLE III. Comparison of second generation interferometers [KAGRA (Somiya, 2011), GEO-HF (Lück et al., 2010), Advanced Virgo (Virgo Collaboration, 2009, 2012), and Advanced LIGO (Harry, 2010; LIGO, 2011)]. The numbers in the seismic row refer to the number of external active, internal passive, and pendulum suspension stages, respectively.

\begin{tabular}{lcccc}
\hline \hline Detector & KAGRA & GEO-HF & Advanced Virgo & Advanced LIGO \\
\hline Arm length $(\mathrm{m})$ & 3000 & 600 & 3000 & 3995 \\
Mirror mass $(\mathrm{kg})$ & 27 & 5.5 & 40 & 40 \\
Beam spot size $(\mathrm{cm})$ & 3.5 & 2.4 & 6 & 5.9 \\
No. of seismic stages & $1+5$ & $1+3+3$ & $1+6$ & $1+2+4$ \\
Stored power $(\mathrm{kW})$ & 400 & 10 & 760 & 800 \\
Strain noise $\left(10^{-23} / \sqrt{\mathrm{Hz}}\right)$ & 0.3 & 3.5 & 0.3 & 0.3 \\
Sensitive band $(\mathrm{kHz})$ & $0.02-3$ & $0.1-5$ & $0.02-3$ & $0.01-5$ \\
Location & Japan & Germany & Italy & USA \\
\hline \hline
\end{tabular}




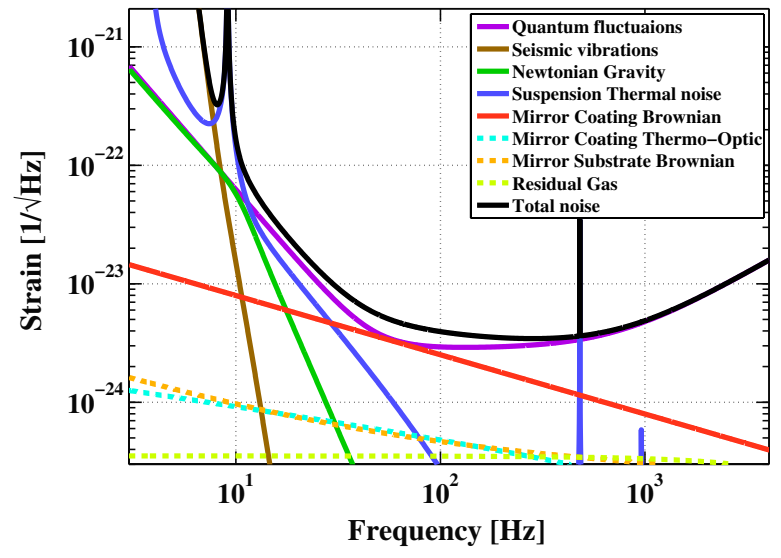

FIG. 13 (color online). Noise budget of the Advanced LIGO interferometers operating in a broadband configuration with the parameters of Table III.

In the individual mirror angle basis, we can define an optical torsional stiffness matrix:

$$
\kappa_{R P}=\frac{2 P}{c} \frac{L}{1-g_{1} g_{2}}\left(\begin{array}{cc}
-g_{2} & 1 \\
1 & -g_{1}
\end{array}\right),
$$

where $P$ is the cavity power, $L$ is the cavity length, and the cavity $g$ factors for each mirror are defined as $g_{i}=1-L / R_{i}$, where $R_{i}$ is the radius of curvature of the $i$ th mirror. The cavity instability occurs when the eigenvalue from this torsional matrix corresponding to the soft mode exceeds the mechanical torsional stiffness of the mirror suspension.

As described in Sec. V.B.1, the cavity beam sizes are maximized to reduce the impact of the mirror's thermal noise. This has the unfortunate side effect of amplifying these optical torsional stiffnesses. The large beam sizes can be realized by utilizing either a plane-parallel or concentric cavity design (Siegman, 1986). As seen from Eq. (18), the concentric design (which has negative $g$ factors) causes the dominant mode to have a positive sign and thereby contribute to the stiff, self-aligning mode. The plane-parallel design, on the other hand, has positive $g$ factors. In this case the

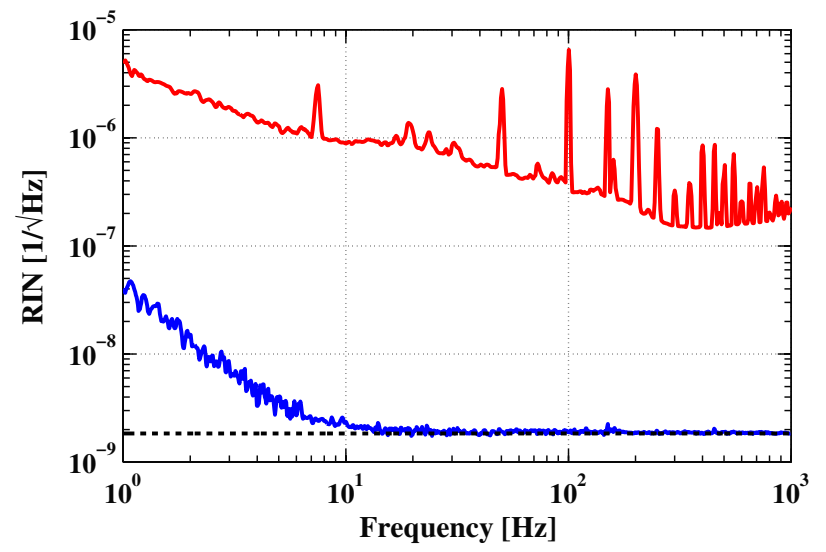

FIG. 14 (color online). Relative power fluctuations after stabilization of a prototype laser system: (top curve) free running laser noise, (bottom curve) stabilized level (out of loop), and (dashed line) shotnoise limit. The goal for Advanced LIGO is $2 \times 10^{-9} / \sqrt{\mathrm{Hz}}$. From Kwee, Willke, and Danzmann, 2009.

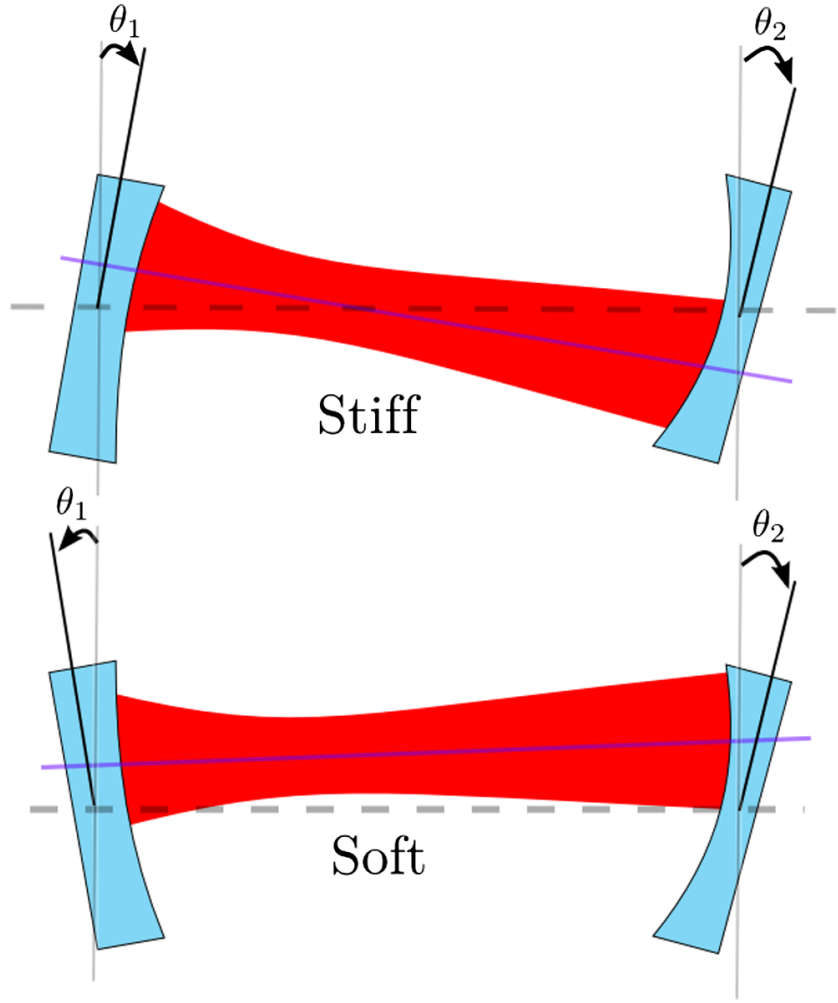

FIG. 15 (color online). The common and differential angular modes of the Fabry-Pérot cavity mirrors are softened (bottom) and stiffened (top) by the radiation pressure torque.

denominator of Eq. (18) blows up as the $g$ factors approach unity (as they must to increase the spot sizes). For this reason, the concentric design has been adopted for all modern GW detectors.

This Sigg-Sidles effect was first characterized for the initial LIGO detectors (Hirose et al., 2010) and then subsequently in the Enhanced LIGO where a modal control approach was used to stabilize it (Dooley, 2011). This modal approach seems to be sufficient to control the instability (Barsotti, Evans, and Fritschel, 2010) but the noise from the control system is likely to be comparable to the more fundamental limits (e.g., suspension thermal noise).

\section{Parametric instabilities}

With high circulating powers in the arm cavities, a parametric instability can occur involving the high- $Q$ mechanical modes of the mirrors and higher-order transverse optical modes of the Fabry-Pérot cavity (Braginsky, Strigin, and Vyatchanin, 2001, 2002; Strigin and Vyatchanin, 2007). Although not observed in the first generation detectors, similar instabilities have been observed in toroidal microcavities (Kippenberg et al., 2005) and in short, kilogram-scale FabryPérot cavities (Corbitt et al., 2006).

Following Evans, Barsotti, and Fritschel (2010), we can write the round-trip parametric gain for the $m$ th mechanical mode as

$$
R_{m}=\frac{4 \pi Q_{m} P}{M \omega_{m}^{2} c \lambda} \sum_{n=0}^{\infty} \Re\left\{G_{n}\right\} B_{m, n}^{2}
$$




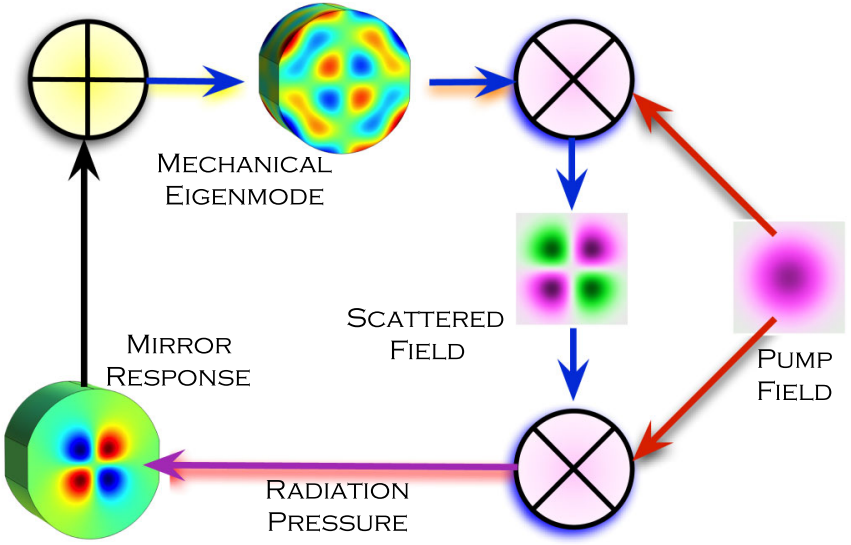

FIG. 16 (color online). Feedback loop diagram of the parametric instability process. The oscillation of one of the mirror's mechanical eigenmodes scatters the resonant cavity mode into a higher-order transverse mode which resonates partially in the coupled optical cavities of the interferometer and returns to excite the mirror via radiation pressure.

where $Q_{m}$ is the mechanical $Q$ of the mode, $P$ is the arm cavity stored power, $M$ is the mirror mass, $\omega_{m}$ is the mechanical eigenfrequency, $\lambda$ is the laser wavelength, $B_{m, n}$ is the overlap coefficient between the mechanical mode and the optical mode, and $G_{n}$ is the round-trip gain for the scattered field within the entire interferometer. This process is shown schematically in Fig. 16.

Even considering the optical resonance of the full interferometer, predicting the impact of parametric instabilities is difficult. The details of the surface figure for each of the mirrors shift the resonant frequency for the higher-order optical modes by a significant fraction of the cavity linewidth. Small differences in dimensions of mirrors and long term drifts in the laboratory temperatures can make order of magnitude changes in the round-trip gain by reducing the frequency overlap between the mechanical and optical modes.

A Monte Carlo analysis (Evans, Barsotti, and Fritschel, 2010) indicates that there is likely to be several unstable modes in a full power Advanced LIGO interferometer. As the masses, $Q$ 's, and power levels are similar, most likely the same problems will afflict the Advanced Virgo and KAGRA interferometers.

Several mitigation strategies have been proposed to suppress these instabilities: adding passive damping films to the mirror "barrel" (Gras, Blair, and Zhao, 2009), attaching a resonant electromechanical damper, active feedback via the existing mirror actuators (Miller et al., 2011), or using the radiation pressure of an external laser (Ju et al., 2009). While there are challenges to be overcome with all of these techniques, it seems likely that a combination of them will be able to suppress the instabilities down to the nuisance level. Future interferometers should be able to scale the mirror mass directly with laser power and thereby stay at a nearly invariant instability level.

\section{E. Low-frequency seismic isolation}

The experience with the initial interferometers highlighted the multitudinous ways in which large, low-frequency

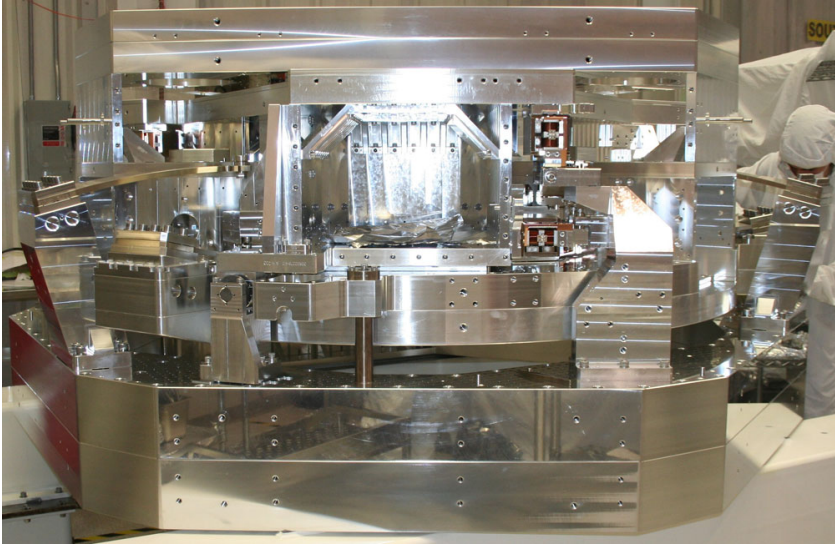

FIG. 17 (color online). Advanced LIGO vibration isolation platform: this double stage, in-vacuum platform provides active isolation from 0.5 to $30 \mathrm{~Hz}$ and passive isolation above $\sim 1 \mathrm{~Hz}$. The leaf springs around the outer edge of the image provide the vertical compliance. The copper coils near the center of the image are part of the coil-magnet actuators used in the active feedback. Inertial sensors in sealed pods are attached to the platforms to provide the readback signals in the isolation servos.

seismic motions can produce noise in the GW signal band through nonlinear upconversion (see Sec. VI.C). As a result, all of the second generation vibration isolation systems seek to reduce motions not only in the GW band, but also in the $0.01-10 \mathrm{~Hz}$ band.

The Advanced LIGO system is a three-stage hybrid, activepassive platform (Abbott et al., 2002). There is a hydraulic preisolator to provide coarse positioning and coarse active vibration control. This is followed by 2 compliant platforms (shown in Fig. 17) which provide passive isolation above $\sim 1 \mathrm{~Hz}$ and active isolation from 0.1 to $30 \mathrm{~Hz}$. An array of seismometers placed near each mirror will be used to reduce the fluctuations in the low-frequency, global interferometric lengths (DeRosa et al., 2012) that arise from the microseismic peaks (Giaime et al., 2003).

A comparison of the vibration isolation performance of all ground-based GW detectors is shown in Fig. 5.

\section{THIRD GENERATION DETECTORS}

Even conservative estimates of astrophysical event rates (Phinney, 1991; Cutler and Thorne, 2002; Abadie et al., 2010) predict many detections per year for the second generation detectors. Once the first detections are well established, one would like to move on to using the waveforms to make tests of astrophysical models, use "standard" sirens for high precision cosmography, and make tests of fundamental physics (Punturo et al., 2010). In order to pursue this type of science, the sensitivity must be pushed beyond what the second generation detectors are capable of.

A combination of astrophysical motivations and technical developments has driven the European design of the Einstein telescope (ET) (Einstein Telescope Science Team, 2011; Sathyaprakash et al., 2012). The ET is foreseen to be an underground, triangular, $10 \mathrm{~km}$ interferometer array operating at cryogenic temperatures. The goal is to improve upon the broadband sensitivity by an order of magnitude over 


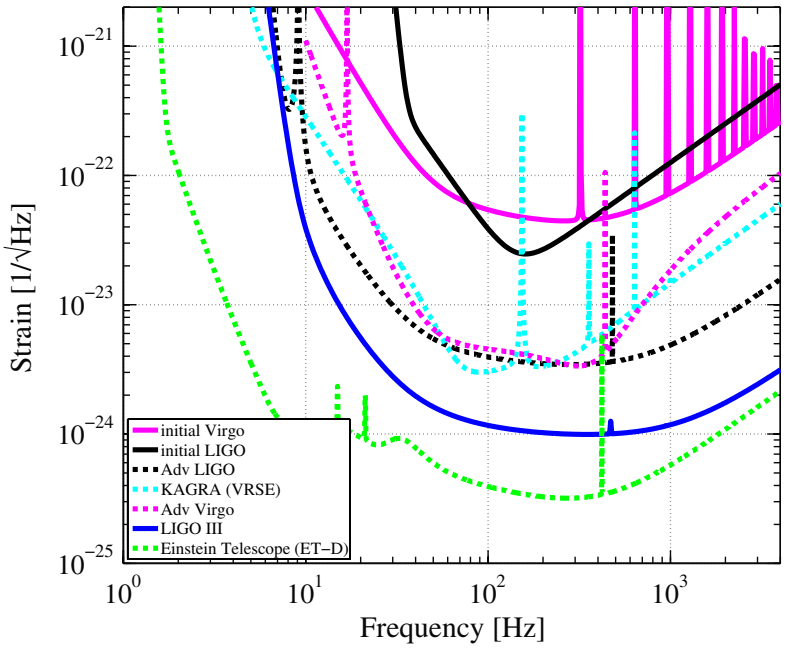

FIG. 18 (color online). Comparison of strain noise estimates for the ground-based detectors. The LIGO-III trace refers to an upgrade of the Advanced LIGO detector including several of the ideas mentioned in Sec. VIII.

the second generation instruments and to lower the low-frequency cutoff by a factor of 2-3. The most recent estimates of the ET sensitivity goal are shown in Fig. 18.

The LIGO Scientific Collaboration is currently studying the possibility of a complementary third generation detector network. As the LIGO detectors have yet to reach the fundamental limits of the existing facilities, the study focuses on implementing the new interferometers in the existing vacuum system.

In the following sections, several of the key techniques to making this improvement (summarized in Fig. 20 and Table IV) are described.

\section{A. Quantum nondemolition}

Most of the noise limits for the large interferometers have already been reduced to below the usual quantum limits (cf. Fig. 13). Improving the quantum limits will give a larger scientific payoff than any other technical improvement. Correspondingly, there has been an explosion of research into QND readout schemes for GW interferometers in the 21 st century. Here we describe several of the most promising ideas (Braginsky and Khalili, 1996).
Recent reviews of the state of the art in QND for GW detectors describe well some of the more promising techniques (Corbitt and Mavalvala, 2004; Schnabel et al., 2010; Chen et al., 2011; McClelland et al., 2011; Chen, 2013). In the past decade, there has been a number of theoretical and experimental advances which have led to better estimates of what is possible. We categorize the basic optical topologies in the following way.

\section{Frequency dependent squeezed state injection}

The injection of squeezed light has long been seen as a panacea for the quantum noise limits of GW detection. However, the direct injection of squeezed light can only reduce the noise in the quadrature which has been squeezed (Caves and Schumaker, 1985; Schumaker and Caves, 1985); phase-squeezed light would improve the shot-noise limited region, but add, at least, a corresponding amount of radiation pressure noise. Work by Kimble et al. (2001) and later by Harms et al. (2003) showed that kilometer sized cavities could be used to apply a frequency dependent phase shift to the squeezed fields. This phase shift can be tuned to provide amplitude squeezing in the band where the radiation pressure is dominant and phase squeezing where the shot noise dominates. For the broadband (tuned-RSE) configuration of Advanced LIGO, this can be accomplished with a single cavity. For the detuned-RSE configurations chosen by Virgo and KAGRA, two cavities are required to optimally match the squeeze quadrature to the interferometer's optomechanical response.

Following early work on producing squeezed states at high frequencies (Wu et al., 1986), the GW community pushed the technology to produce high levels of squeezing at audio frequencies (McKenzie et al., 2004; Vahlbruch et al., 2006, 2007; Mckenzie, 2008; Chua et al., 2011; Stefszky et al., 2012) on tabletop prototypes. In the last few years, moderate levels of noise improvement have been observed from injecting squeezed light into a suspended prototype (Goda et al., 2008) as well as the GEO600 (LIGO Scientific Collaboration, 2011) and Enhanced LIGO (Aasi et al., 2013; Dwyer, 2013) detectors.

With the confidence gained from these demonstrations and the imminent prototyping of quadrature rotating filter cavities, it is very likely that the second generation detectors can be upgraded with the injection of squeezed states of light before the end of the decade.

TABLE IV. Nominal values of some LIGO-III interferometer parameters used for Fig. 20.

\begin{tabular}{|c|c|c|c|c|c|c|c|}
\hline Parameter & Symbol & Value & Units & Parameter & Symbol & Value & Units \\
\hline Light wavelength & $\lambda$ & 1064 & $\mathrm{~nm}$ & Substrate Young's modulus & $Y_{\text {sub }}$ & 185 & $\overline{\mathrm{GPa}}$ \\
\hline Arm cavity mirror mass & $m$ & 145 & $\mathrm{~kg}$ & Suspension ribbon Young's modulus & $Y_{s i}$ & 130 & $\mathrm{GPa}$ \\
\hline Arm cavity length & $L$ & 4000 & $\mathrm{~m}$ & Suspension ribbon thickness & $h_{\mathrm{sus}}$ & 0.2 & $\mathrm{~mm}$ \\
\hline Arm cavity finesse & $F$ & 550 & $\cdots$ & Suspension ribbon width & $d_{\text {sus }}$ & 2 & $\mathrm{~mm}$ \\
\hline Arm cavity power & $P_{\text {cav }}$ & 3000 & $\mathrm{~kW}$ & Substrate loss angle & $\phi_{\text {sub }}$ & $3 \times 10^{-9}$ & $\mathrm{rad}$ \\
\hline Beam radius & $\omega$ & 5.8 & $\mathrm{~cm}$ & Coating loss angle & $\phi_{\text {coat }}$ & $2 \times 10^{-5}$ & $\mathrm{rad}$ \\
\hline Detection efficiency & $\eta$ & 0.95 & $\cdots$ & Mirror coating & $\cdots$ & GaAs:AlAs & $\cdots$ \\
\hline Squeeze factor & $R$ & 10 & $\mathrm{~dB}$ & & & & \\
\hline Filter cavity length & $L_{\mathrm{fc}}$ & 100 & $\mathrm{~m}$ & Newtonian noise subtraction factor & $\kappa_{\mathrm{NN}}$ & 30 & . . \\
\hline Filter cavity loss & $A_{\mathrm{fc}}$ & 33 & ppm & Mirror or suspension temperature & $T$ & 120 & K \\
\hline
\end{tabular}




\section{Frequency dependent readout quadrature}

The quantum correlations built up in the signal recycled interferometers make it possible to surpass the standard quantum Limit in a narrow band (Vyatchanin and Matsko, 1996; Buonanno and Chen, 2002). At high-power levels, the vacuum fields in the amplitude quadrature drive the mirror and produce signals in the phase quadrature as well. By choosing the appropriate combination of homodyne readout quadratures after the photodetection, the amplitude noise can be partially canceled.

The addition of one of the long quadrature rotation cavities allows one to rotate the readout quadrature as a function of frequency and cancel the sensitivity to the radiation pressure noise (Kimble et al., 2001; Khalili, 2007). The radiation pressure noise itself has not been canceled; the mirrors are still moving. Rather, we have chosen to adjust the phase of our optical readout so as to ignore such perturbations. This technique is often referred to as the variational readout technique. However, this delicate cancellation by tuning of the readout quadrature has its problems: optical losses in the rotation cavity degrade this scheme faster than the squeezed light injection scheme above. Since the cancellation also subtracts much of the signal, it becomes more sensitive to any degradation of the internal squeezing due to losses.

\section{QND observable readout}

In the Heisenberg picture, the increase in our positional resolution comes at the expense of increased momentum perturbations since the position and momentum do not commute. The momentum perturbations influence the time evolution of the mirror position and spoil the low-frequency sensitivity. An alternative to this approach is to read out some observable which carries the gravitational-wave information and also commutes with itself at later times. In this way, we have a true QND observable readout and not have to worry about the quantum backaction effects (Braginsky and Khalili, 1999). One such observable is the mirror's momentum (or speed) (Braginsky et al., 2000; Purdue and Chen, 2002; Chen, 2003). Practically, this can be done by adding one of the long filter cavities into the interferometer in such a way so as to differentiate the usual positional signal. By taking the differences between successive position measurements, the readout variable closely approximates momentum and so this type of interferometer is often referred to as a speed meter.

\section{Optical losses and QND}

In addition to the squeezing input, the variational readout, and the speed meter, there are a host of other possibilities for QND upgrades: optical "levers" (Khalili, 2002), multiwavelength optical springs (Rehbein et al., 2008), and multiwavelength "xylophones" (Rehbein et al., 2007), etc. The issues with most of the previous intercomparisons is that they do not include losses in a realistic way. In addition to the optical losses due to scattering within the interferometer, losses in the readout chain, and finite quantum efficiency of photodetectors, one must also include the losses in the quadrature rotating filter cavities. To include these losses in a realistic way, it is important to remember that the true loss will scale

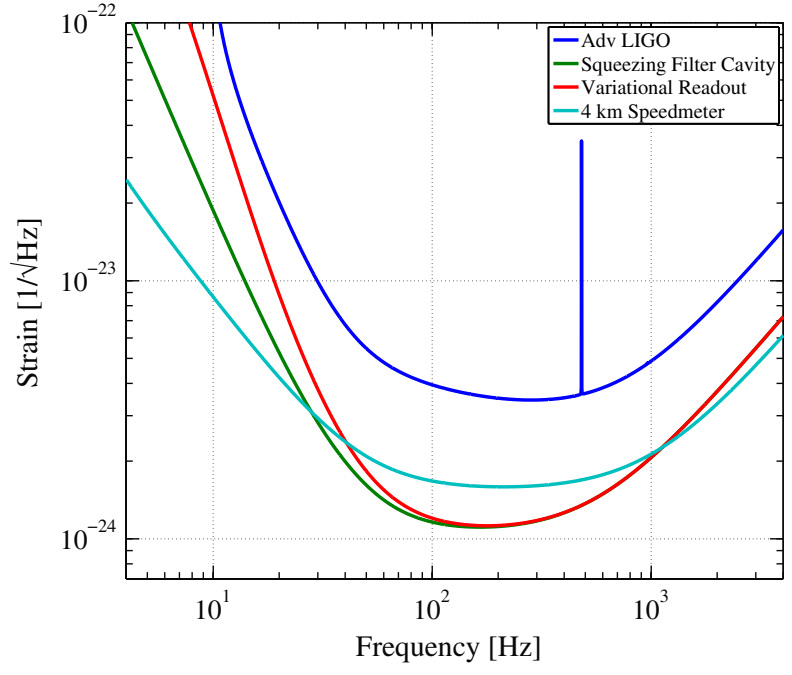

FIG. 19 (color online). Comparison of the equivalent strain noise levels of various quantum nondemolition schemes implemented on this LIGO-III concept. $10 \mathrm{~dB}$ squeezed light is injected in all cases. For the "Filter Cavity" trace, squeezed light is filtered with a $100 \mathrm{~m}$ cavity. For the "Variational" trace, the output of the interferometer is filtered by a $100 \mathrm{~m}$ filter cavity, and for the speed-meter case, the "Filter Cavity" configuration is modified by adding a $4 \mathrm{~km}$ speedmeter cavity.

with the beam size (cf. Sec. VII.B) and thereby the cavity length.

A numerical comparison that incorporates realistic losses within the framework of the "LIGO-III" design has been carried out by Miao, Chen, and Adhikari (2013) and is shown in Fig. 19. Here it has been assumed that the round-trip losses in the filter cavity are $33 \mathrm{ppm}$ (consistent with the past experience with large cavities).

\section{B. Circumventing mirror thermal noise}

The relatively large mechanical dissipation in the mirror coatings and the shallow frequency dependence of the resulting mirror surface fluctuations makes the coating thermal noise one of the most serious limits for future detectors. Broadly speaking, two approaches are being pursued to avoid this limit: new coatings with higher mechanical $Q$ 's and alternative optical cavity mode shapes that can partially reject the noise.

\section{Non-Gaussian beam shapes}

A straightforward approach to reducing the effects of coating thermal noise is to increase the beam size (cf. Sec. V.B.1). However, as described in Sec. VII.D.1, this can exacerbate the radiation pressure induced angular instability. Even if this can be compensated by an exceptionally sophisticated feedback control system, it is unlikely that beam size alone will offer more than a factor of 2 improvement in the long run.

A more effective approach might be using higher-order spatial modes of the cavity. The field inside of a Fabry-Pérot cavity with spherical mirrors can be well approximated with the set of orthonormal Laguerre-Gaussian functions (Siegman, 1986). Within the Virgo project (Vinet, 2010), it 
has been proposed to use axially symmetric LaguerreGaussian modes (chiefly the $\mathrm{LG}_{3,3}$ and the $\mathrm{LG}_{5,5}$ modes).

In addition to the technical difficulties associated with the generation (Granata, Buy et al., 2010) and control (Chelkowski, Hild, and Freise, 2009) of such beams, a stability analysis of the cavities (Hong et al., 2011) shows that the cavity field is strongly distorted when taking into account the realistic surface imperfections (see Fig. 10) of the best available mirrors. The several-fold degeneracy of these higherorder modes is weakly split by the surface deformations and all of the degenerate modes are partially resonant. In this perturbed state, the fields from the two arm cavities are no longer well matched and this degrades the interference at the antisymmetric port of the Michelson. Consequently, the ability to make a low phase noise optical readout is compromised.

An even more complicated option is to use a particular linear combination of Laguerre-Gaussian modes. The socalled "Mesa beams" (D'Ambrosio et al., 2004; Tarallo et al., 2007; Miller et al., 2008) are one such combination. Simulations (Hong et al., 2011) show that they are not much worse than $\mathrm{TEM}_{0,0}$ Gaussian modes in their susceptibility to angular instabilities or modal degeneracy. Unfortunately, it is not yet straightforward to produce the nonspherical mirrors required for Mesa beams.

The theoretical maximum improvement from any of the above beam shaping techniques is $\sim 70 \%$. To make any further improvements it will be necessary to either have radical improvements in the mechanical loss of mirror coatings or build a much longer interferometer.

\section{Heteroepitaxial Bragg mirrors}

As described in Sec. V.B.1, the thermal fluctuations of the mirror surface are dominated by the Langevin thermal forces generated in the high-reflectivity dielectric coatings. It has been shown (Phillips, 1987; Pohl, Liu, and Thompson, 2002) that the mechanical dissipation (and consequently the thermal noise) of nearly all amorphous, thin-film materials is higher than that of crystalline materials. The cause of the dissipation, almost universally, is known to be due to the presence of a set of low energy modes (which are not "frozen out"). Tunneling into this vast sea of available modes leads to the observed mechanical dissipation. Of all amorphous solids, fused silica seems to be singular in its extremely low dissipation at room temperature and above (Ageev et al., 2004). Unfortunately, this high $Q$ of the bulk material does not translate into high $Q$ for the silica thin films used in the optics industry.

One strategy in avoiding this thermal noise source is to eschew coatings altogether and to use corner reflectors (Braginsky and Vyatchanin, 2004; Cella and Giazotto, 2006) or total internal reflection (Schiller et al., 1992; Goßler et al., 2007). Although these approaches introduce new technical problems, there is, so far, no known fundamental reason why they cannot be used to supplant coatings. A rigorous theoretical treatment followed by a direct thermal noise experiment is required.

Another approach to avoiding mechanically lossy coatings is to pattern the surface of the substrate in order to produce grating based waveguide reflectors (Bunkowski et al., 2006). Work in this area has resulted in impressive performance in recent years (Brückner et al., 2008; Friedrich et al., 2011;
Kroker et al., 2011) approaching power reflectivities of up to $99.9 \%$. Incorporating gratings into interferometers for gravitational-wave detection require substantial hurdles to be overcome: the coupling of mirror alignment fluctuations (Freise, Bunkowski, and Schnabel, 2007; Kroker et al., 2013) and transverse mirror motions (Wise et al., 2005; Brown et al., 2013) into longitudinal phase noise, the control of microroughness to reduce the diffuse scattered light (Woods et al., 1994; Magaña-Sandoval et al., 2012), the control of the large scale flatness to control the mirror figure error, and reducing the transmission losses by another factor of $10(R=$ $99.999 \%$ ).

A less exotic option is to search more widely for lower mechanical loss materials which can produce Bragg reflectors in the same manner as is done with the standard dielectric coatings. The poor mechanical $Q$ of amorphous materials leads one toward crystalline coatings. Epitaxial deposition techniques (e.g., chemical vapor deposition, molecular beam epitaxy, atomic layer deposition) have advanced dramatically over the past several decades to support the development of electronic circuits and optoelectronics.

A promising set of prospects are trinary AlGaAs layers grown on GaAs substrates and then attached to silica or silicon mirrors via epitaxial liftoff (ELO) (Demeester et al., 1993). These structures have been grown on GaAs substrates and the resulting mechanical $Q$ is $\sim 30$ times larger than the best amorphous high-reflectivity coatings (Cole et al., 2008). Another possibility is to grow AlGaP:GaP (Lin, Harris, and Fejer, 2011) layers directly onto silicon substrates where the lattice matching is quite good. The matching may largely mitigate the thermal stresses and allow operation of the interferometer at cryogenic temperatures where the thermal noise is further reduced. If the mechanical dissipation can be maintained at such low levels after ELO and the absorption can be reduced to $\lesssim 5 \mathrm{ppm}$, these epitaxial coatings have the promise of expanding the astrophysical reach of the detectors by a factor of 3-10 in the most critical frequency band.

\section{Newtonian gravity noise subtraction}

As described in Sec. V.B.4, the fluctuations in the local gravitational field will limit any further progress below $\sim 20 \mathrm{~Hz}$ due to the inability to shield the mirrors from local gravitational perturbations. The seismic noise shown in Fig. 4 indicates that the situation is largely the same for LIGO and Virgo, while the Newtonian noise may be as much as an order of magnitude smaller for KAGRA. Underground detectors such as KAGRA and the Einstein telescope should be designed to have a high degree of symmetry in the shape of the caverns around each test mass. The symmetry of these caverns can then passively cancel much of the Newtonian gravity noise (Cella, 2006; Harms et al., 2009). Although it is not possible to significantly reduce the ambient vibrations, it is possible, in principle, to subtract this gravitational noise either by applying canceling forces on the mirrors or by regressing it from the data stream offline.

Clearly the major impediment to subtracting out noise sources, in general, is to determine what part of the interferometer output is noise and what part is signal. If this was straightforward, then all of the important noises could be 


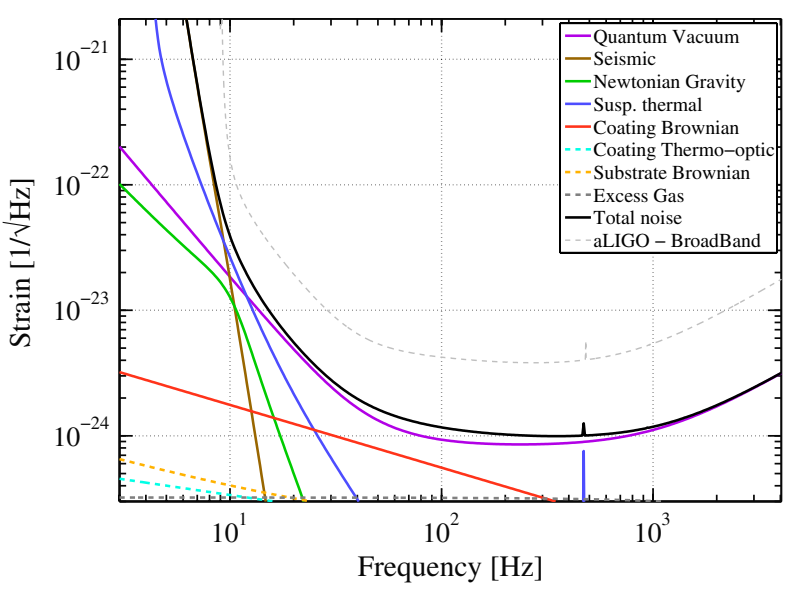

FIG. 20 (color online). Limiting noise sources for a potential third generation LIGO detector with $3 \mathrm{MW}$ of arm cavity power, $10 \mathrm{~dB}$ of frequency dependent squeezed light injection, $140 \mathrm{~kg} \mathrm{Si}$ mirrors with GaAs coatings operating cryogenically at $120 \mathrm{~K}$, and $30 \times$ subtraction of Newtonian gravity noise.

removed in this manner. The distinguishing feature of the Newtonian noise, however, is that the source terms are readily measured. As seen from Fig. 6, the dominant component comes from the ambient ground motion in the vicinity of the test masses. Of the various modes of the ground, the chief contributors to the gravitational noise are the Rayleigh waves (Beccaria et al., 1998b; Hughes and Thorne, 1998) on the surface. The body waves in the ground produce only small density perturbations and are at least 10 times smaller in their gravitational impact.

In principle, an array of seismometers near each mirror could measure this surface wave contribution. Given the time series of seismic noise, the remaining step is to then determine the Green's function that relates the motion of each sensor to the mirror motion. Given sufficient knowledge about the ground and the surrounding laboratory environment this could possibly give some moderate subtraction quality, but would require significant effort to perform the characterization and construct such an elaborate model with any accuracy.

A more promising approach is to use adaptive noise cancellation algorithms to "learn" the Green's function and apply the resulting digital filters to the data stream (Haykin, 2002; Sayed, 2003; Huang, Benesty, and Chen, 2006; Beker et al., 2011). This approach has proven to be successful in the laboratory (Thorpe et al., 2010; Driggers et al., 2012; Leibrandt, Bergquist, and Rosenband, 2013) in subtracting the direct seismic influences from fixed cavities and suspended interferometers using an array of several low noise seismometers and accelerometers. Moreover, this technique was employed in the recent LIGO science run (S6) to remove the seismic influence from several of the interferometric degrees of freedom (DeRosa et al., 2012) as well as magnetic field fluctuations at the main frequencies (Fricke et al., 2012).

Early estimates of the noise at the LIGO sites and simulations of the subtraction systems indicate that an array (shown in Fig. 21) of $\sim 10-20$ sensors per test mass will be sufficient to subtract $90 \%$ of the noise in the $5-20 \mathrm{~Hz}$ band (Driggers, Harms, and Adhikari, 2012). Experience with Advanced

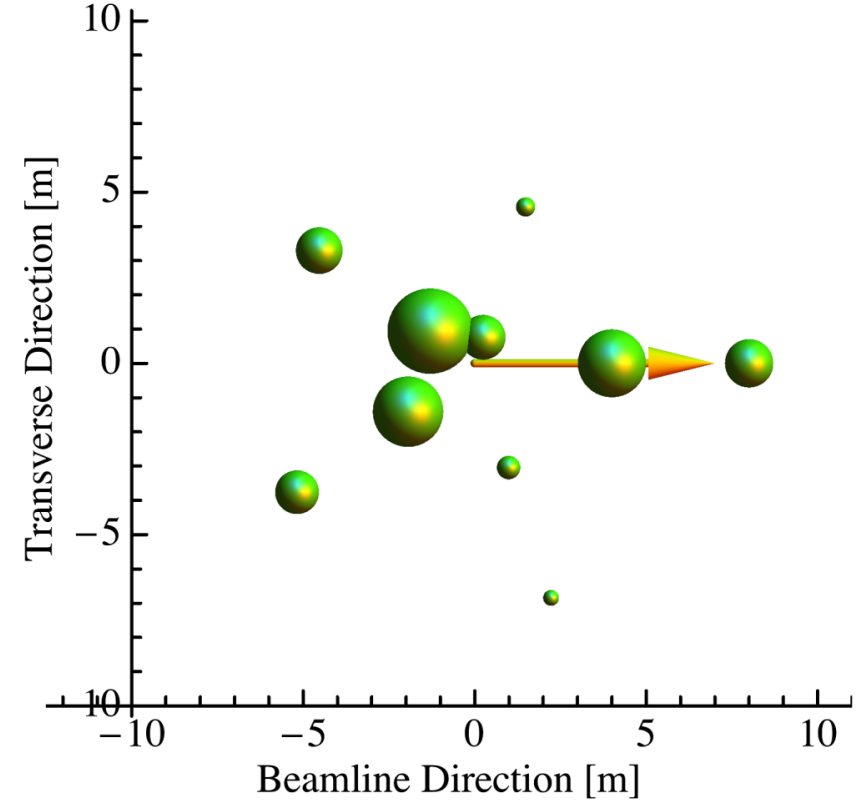

FIG. 21 (color online). An example spiral array optimized for subtracting Newtonian noise due to surface waves for the LIGO-III concept shown in Fig. 20. The center of each sphere indicates the location of one seismometer and the size of the sphere is proportional to the coherence between the seismometer and the Newtonian gravitational perturbations on the test mass. Here the test mass is at $(0,0)$ and the laser beam direction is indicated by the arrow. From Driggers, Harms, and Adhikari, 2012.

LIGO should allow for making improvements to this depending upon the complexity of the seismic fields.

At the low frequencies where Newtonian noise is dominant, the main sources of gravitational waves are expected to be the mergers of intermediate mass $\left[M \simeq(10-1000) M_{\odot}\right]$ black holes and the early part of the inspiral for solar mass compact binaries. The implementation of a Newtonian noise subtraction system should eventually allow for localizing the solar mass binaries to within a reasonable window in the sky and allow electromagnetic telescopes to point to the source well ahead of the merger.

\section{Beyond silica suspensions}

In the third generation mirror suspensions, the frontier is not in improving the vibration isolation, but rather it is in reducing the thermal noise due to the suspension fiber. As discussed in Sec. VII.A, the present limit to the mechanical dissipation comes from the residual defects in the surface layer of the silica fibers. From Eqs. (11) and (13) we can see that progress can be made on two fronts: reducing the temperature and reducing the loss.

\section{Silicon suspensions and cryogenics}

To reduce the loss it will be necessary to use a material with a very high mechanical $Q$ [e.g., sapphire, silicon, niobium, diamond (Gaidarzhy et al., 2007)] as well as an extremely high-quality surface. Fortunately, surface treatments of monocrystalline silicon have advanced dramatically over the past decade. Present-day technologies can already 
produce silicon with 10 times less surface loss than fused silica (Nawrodt et al., 2010) and new etching and passivation methods pioneered by the optomechanics community (Borselli, Johnson, and Painter, 2006) may surpass this limit by another order of magnitude in the coming decade.

A seemingly straightforward option is to simply operate the interferometer at cryogenic temperatures, thereby winning in the thermal noise as $T^{-1 / 2}$. In addition, cryogenic silicon has many other excellent low temperature properties: the thermal expansion coefficient goes through zero at 18 and $120 \mathrm{~K}$, the thermal conductivity is at least 300 times higher than that of silica (thus reducing thermal gradients and distortions), and, as is the case with many crystalline substances, the mechanical $Q$ increases with decreasing temperature.

With MW level laser power in the Fabry-Pérot cavities, extracting heat from the mirrors becomes an issue for low temperature operation. The high thermal conductivity of materials such as silicon and sapphire may make it possible to extract $\sim 10 \mathrm{~mW}$ of heat through the suspension fibers (Tomaru et al., 2002) before the thickness of the fiber compromises the thermal noise benefits.

At the higher zero crossing temperature of $120 \mathrm{~K}$, the radiative cooling power of a large mirror can exceed $\sim 10 \mathrm{~W}$. This should make it possible to cool the mirrors entirely by radiation using a cold shield around the suspension. This essentially noiseless approach should permit the use of 10 times higher circulating power in the interferometer while maintaining the thermal noise benefits of low temperature operation. It remains to be seen if the surfaces can be treated in a way so as to have a high emissivity while not spoiling the mechanical $Q$ too much.

\section{Electromagnetic suspensions}

A natural route to explore is that of eschewing the fiber altogether and using purely magnetic suspension forces (Jayawant, 1981; Drever, 1996). In principle, the lack of any mechanical support element will eliminate the suspension thermal noise contribution to the interferometer displacement noise. Attaching magnets directly to the mirror is likely to lead to too much mechanical dissipation in the attachments and in the magnets themselves. Another possibility is to find a paramagnetic mirror material and to levitate it with strong permanent magnets (Augst and Drever, 2000), although it seems problematic to simultaneously have a large magnetic susceptibility and high mechanical $Q$. In either case, the mirror must be well shielded from the ambient magnetic field fluctuations and even then, the Barkhausen noise in the permanent magnets could well introduce an insurmountable noise floor. Even superconducting magnetic suspensions may have dissipation (Hebard, 1973; Hammond et al., 2004) due to nearby eddy currents or small normal regions of the material.

Rather than directly levitating the mirror, the magnetic suspension could be used to support an upper stage of a multistage suspension system (Varvella et al., 2004). The mirror could then be supported from this magnetically levitated platform by a passive mechanical suspension. Although this approach would not avoid the fiber's thermal noise, it could allow for a very low-frequency suspension and concomitant improvement in filtering of seismic noise.
Another option is to instead use electrostatic (Giazotto, 1998; Willemenot and Touboul, 2000) suspensions. This would seemingly avoid the problems due to coupling from ambient magnetic fields. In any case, the passive stability of any such system is forbidden by Earnshaw's theorem, and some kind of active feedback must be used to stabilize at least 1 degree of freedom. The sensitivity of such a sensor limits the ultimate low-frequency performance of such a suspension-levitation system, but it may be very useful as an intermediate stage in a compound pendulum system.

\section{LOW-FREQUENCY DETECTORS}

The gravitational-wave spectrum spans 20 decades in frequency: at the lowest frequencies, corresponding to the age of the Universe, the polarization of the cosmic microwave background should contain signals from the primordial gravitational waves due to cosmic inflation $(\mathrm{Hu}$ and Dodelson, 2002). The nano- to micro-Hertz band is covered by timing of pulsars and artificial satellites (cf. Sec. III.B). Between the timing measurements and the ground-based detectors, the wide $10^{-5}-1 \mathrm{~Hz}$ band will be pursued with space-based interferometers in the near future.

\section{A. Interferometers in space}

Space detectors have tremendous advantages over groundbased detectors below $\sim 5 \mathrm{~Hz}$. Direct seismic vibrations and Newtonian gravitational fluctuations are almost completely absent. All of the proposed space missions, therefore, are designed to focus on sub- $\mathrm{Hz}$ frequencies.

\section{1. eLISA}

eLISA (previously LISA: the Laser Interferometer Space Antenna) is a proposed European Space Agency variant (Danzmann, 2013) of LISA (Faller et al., 1989; Prince et al., 2002; Danzmann and Rüdiger, 2003; Prince and LISA Science Team, 2009) slated for launch in the early part of the 21 st century. The design has three spacecraft flying in a near-equilateral triangle formation in orbit around the Sun, trailing the Earth by $\sim 20^{\circ}$. Whereas the original LISA mission had links between each of the spacecraft, the new eLISA concept has two interferometric links. From the central satellite, a laser beam is sent to the others using a large beam expanding telescope. Because of the large distances, most of the light is lost through diffraction, yielding very little power for detection at each end. The local lasers at each receiving satellite are then phase locked to the incoming light. The local laser light is then sent to the central satellite similar to standard transponder methods. The phase differences between the lasers contain the GW strain signal as well as various technical noise sources which can be removed by the techniques of time-delay interferometry (Armstrong, Estabrook, and Tinto, 1999; Tinto and Dhurandhar, 2005; de Vine et al., 2010). The low power levels which are received at each satellite result in the interferometer being shot noise limited above a few $\mathrm{mHz}$. Although the expected displacement sensitivity is "only" $\sim 10^{-11} \mathrm{~m} / \sqrt{\mathrm{Hz}}$, the impressive strain sensitivity is achieved by having arm lengths of $\sim 10^{6} \mathrm{~km}$. In contrast to the ground-based detectors, 


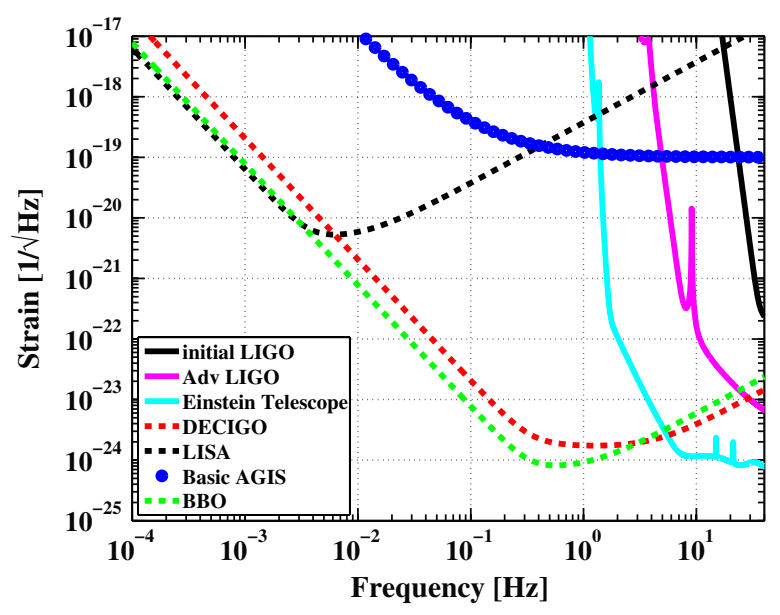

FIG. 22 (color online). Comparison of strain noise estimates for future detectors: LISA, DECIGO, BBO, Basic AGIS, and ET (D). The LIGO sensitivity curves are included for reference.

eLISA will operate in the limit of having many high SNR signals enabling it to do extremely precise tests of astrophysical models and general relativity. In fact, the high sensitivity is expected to lead to a so-called "confusion noise" limit (Królak, Tinto, and Vallisneri, 2004) where the lowfrequency end of the spectrum is dominated by a large foreground of gravitational radiation from galactic and extragalactic compact binaries. In order to reach the sensitivity shown in Fig. 22, sophisticated subtraction algorithms will have to be used in postprocessing (Cornish and Porter, 2007). The rotation of the eLISA constellation and its orbit around the Sun will produce phase and amplitude modulations of the detected signals. These modulations in turn will allow the analysis to reconstruct the angular position of the sources with orders-of-magnitude better resolution than the groundbased detectors. Details of the mission technology and science goals can be found in the eLISA Yellow Book (NGO, 2013).

\section{DECIGO and BBO}

Of all of the proposed sources of gravitational radiation, the most exciting one for cosmologists is perhaps the early Universe (cf. Sec. II.B.3). Because of the weak coupling of gravitational waves with matter, a detection of a primordial stochastic background would allow us to peer back into the time when the age of the Universe was less than $\sim 10^{-20} \mathrm{~s}$. For a scale invariant spectrum of radiation, we have the best chance of detection at low frequencies. Unfortunately, the astrophysical foreground of gravitational waves in the $10^{-9}-10^{-1} \mathrm{~Hz}$ band makes the detection of an inflationary background nearly hopeless. Nearly all of the white dwarf binaries have merged before their orbital frequencies have increased to $0.1 \mathrm{~Hz}$ (Farmer and Phinney, 2003) and so only a relatively small number of binaries containing neutron stars and black holes remain in the $0.1-1 \mathrm{~Hz}$ band.

Two space missions are being studied to probe this frequency band: the Japanese Deci-Hertz Gravitational-wave Observatory (DECIGO) (Ando et al., 2010; DECIGO, 2011) and the international Big Bang Observer (BBO)

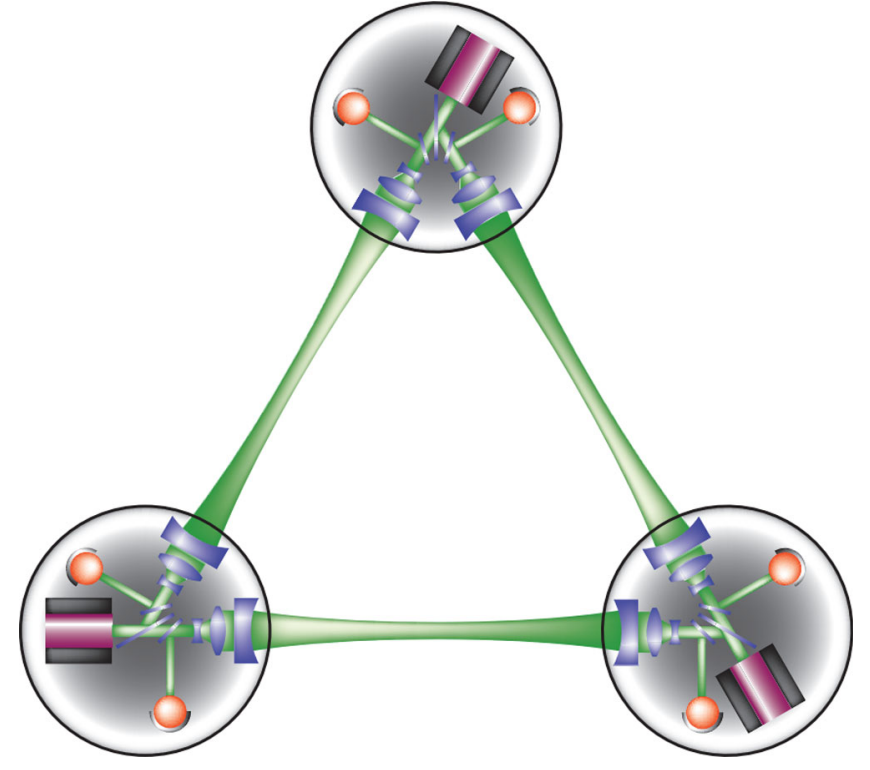

FIG. 23 (color online). DECIGO constellation concept. From Sato et al., 2009.

(Phinney, 2003; Cutler and Harms, 2006). In addition to the eventual detection of cosmological backgrounds, there is a wealth of astrophysical science which can be extracted during the foreground removal of these detectors (Cutler and Holz, 2009; Yagi and Tanaka, 2010). Unfortunately, it is unlikely that either of these missions will fly within the next decade due to budgetary constraints. A three constellation concept which is common to DECIGO, BBO, and the early versions of LISA/eLISA is shown in Fig. 23.

\section{B. Low-frequency terrestrial detectors}

The natural way to avoid terrestrial disturbances is to make an extraterrestrial detector. However, recent advances in our understanding of Newtonian gravitational noise have made it reasonable to reexamine this issue. In particular, the relatively higher abundance of sources in the $0.01-10 \mathrm{~Hz}$ band and their long duration make it possible to have astrophysically interesting detectors even if their noise floors are higher by a factor of $10^{5}$ than that of the km-scale, ground-based detectors (Harms et al., 2013).

\section{Torsion bar antenna}

Recently, a novel arrangement of torsion bars has been proposed to readout sub- $\mathrm{Hz}$ gravitational waves (Ando, Ishidoshiro et al., 2010; Ishidoshiro et al., 2011). The tidal force from an incoming wave will twist the crossed torsion bars differentially. A high sensitivity interferometric sensor is used to read out the differential torsion angle. Early estimates project the strain sensitivity to be near $10^{-19} / \sqrt{\mathrm{Hz}}$ above $\sim 0.1 \mathrm{~Hz}$ using $10 \mathrm{~m}$ bars. With such a sensitivity it should be possible to observe the mergers of intermediate mass black holes out to cosmological distances, search for the merger of galactic white dwarfs (Farmer and Phinney, 2003), and serve as an early warning system for extragalactic compact object inspirals for the ground-based detectors. 


\section{Atom interferometers}

An alternative to standard laser interferometry is to use clouds of atoms instead of mirrors (Dimopoulos et al., 2008; Hohensee et al., 2011). This method uses pulses of light to change the momentum states of some of the atoms in the clouds. These clouds then take different free fall paths. A final pulse is used to synchronize the momentum states of the atoms and the interference of the atomic clouds is used to read out the GW signal.

The advantages of these atomic techniques are many: the clouds have a very high immunity to radiation pressure noise, very low thermal noise, and no suspension noise. The common launch for the atomic clouds makes the influence of seismic noise nearly zero. However, the Newtonian noise is a problem for the atom interferometers just as it is for laser interferometers. A spaced-based detector, Atomic Gravitational wave Interferometric Sensor (AGIS), has also been proposed to circumvent these terrestrial limits (Hogan et al., 2011).

Bender (2011, 2012) highlighted several additional complications (including wavefront aberration and beam jitter) with the light-pulse atom interferometers which significantly limit the achievable sensitivity; these issues are being addressed by the atomic community (Dimopoulos et al., 2011) It remains to be seen if this type of atom interferometry can be made to be competitive with other technologies (such as DECIGO).

\section{CONCLUSION}

Many of the most interesting objects in the Universe remain invisible so far to those of us on Earth. Our understanding of astrophysics and cosmology has been transformed in the past millennium by observations of electromagnetic radiation, looking into new wavelengths, looking farther back into the early Universe, and looking more deeply at our local neighborhood.

We have yet to witness the same revolution through our observations of gravitational radiation, and yet the promise for discovery and revolution remains as profound as before.

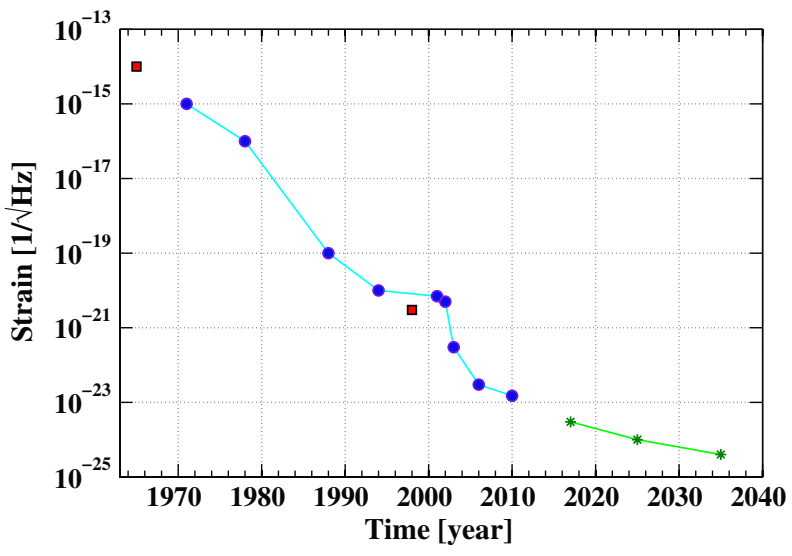

FIG. 24 (color online). Evolution in gravitational-wave detector sensitivity from 1965 into the near future. The minimum of the strain noise spectral density for the given detectors is plotted on the $y$ axis. The acoustic bar detectors are shown as squares and the laser interferometers as circles. Estimates for future detectors are stars.
The recent progress in numerical relativity, wide area astronomical surveys, and gravitational-wave detector technology (shown in Fig. 24) all point to a convergence of science that will ineluctably lead to another series of revolutions in our understanding of the Universe.

The upcoming crop of ground-based detectors is almost guaranteed to make detection in the next few years and the laboratory research of today promises to turn the gravitational-wave astronomy of the future into a precision science. Buoyed by the likely detections of signals by pulsar timing and terrestrial interferometers, the space missions should complete our coverage of the gravitational-wave spectrum. The sources of gravitational waves may often be dark but the future is bright.

\section{ACKNOWLEDGMENTS}

I thank the international community of scientists involved in the search for gravitational waves; I have learned much from them in the last decade. In particular, I thank K. Arai, L. Barsotti, M. Barsuglia, V. Frolov, H. Grote, H. Lück, J. Marque, D. Tatsumi, and G. Vajente for detailed information on the first generation interferometers and their noise sources. I am grateful to M. Ando, Y. Aso, P. Fritschel, S. Hild, and G. Losurdo for detailed information on the second generation interferometers. I must also thank M. Coughlin, J.C. Driggers, and J. Harms for assembling the updated seismic information from the worldwide network and producing the recent estimates of Newtonian gravity noise, P. Kwee for the information on the AEI $200 \mathrm{~W}$ laser, C. Vorvick for images of the LIGO optic scattering, T. Akutsu for information on DECIGO, W. W. Johnson for elucidation on the sensitivity of bar detectors, S. E. Whitcomb for discussions about excess gas, and E. K. Gustafson and K. Arai for a careful reading of the manuscript. I gratefully acknowledge the support of the National Science Foundation under Grant No. PHY-0555406.

\section{REFERENCES}

Aasi, J., et al., 2013, Nat. Photonics 7, 613.

Abadie, J., et al., 2010, Classical Quantum Gravity 27, 173001.

Abbott, B., et al., 2004, Nucl. Instrum. Methods Phys. Res., Sect. A 517, 154.

Abbott, B., et al. (LIGO Scientific Collaboration), 2008, Phys. Rev. Lett. 101, 211102.

Abbott, B.P., et al., 2009a, Nature (London) 460, 990.

Abbott, B. P., et al., 2009b, Phys. Rev. Lett. 102, 111102.

Abbott, B. P., et al., 2009c, Rep. Prog. Phys. 72, 076901.

Abbott, L., and M. B. Wise, 1984, Nucl. Phys. B244, 541.

Abbott, R., et al., 2002, Classical Quantum Gravity 19, 1591.

Abbott, R., et al., 2004, Classical Quantum Gravity 21, S915.

Abramovici, A., et al., 1992, Science 256, 325.

Accadia, T., F. Acernese, F. Antonucci, P. Astone, G. Ballardin, F. Barone, M. Barsuglia, A. Basti, T. Bauer, and M. G. Beker, 2011, Astropart. Phys. 34, 327.

Accadia, T., B. L. Swinkels, and V. Collaboration, 2010, Classical Quantum Gravity 27, 084002.

Accadia, T., et al., 2011a, Astropart. Phys. 34, 521.

Accadia, T., et al., 2011b, Low Frequency Noise, Vibration and Active Control 30, 63.

Accadia, T., et al., 2012a, JINST 7, P03012.

Accadia, T., et al., 2012b, Classical Quantum Gravity 29, 025005. 
Acernese, F., et al., 2004, Classical Quantum Gravity 21, S433.

Acernese, F., et al., 2006a, IEEE Trans. Instrum. Meas. 55, 1985.

Acernese, F., et al., 2006b, Classical Quantum Gravity 23, S91.

Acernese, F., et al., 2007, Appl. Opt. 46, 3466.

Acernese, F., et al., 2008, Classical Quantum Gravity 25, 184001.

Acernese, F., et al., 2009, Phys. Rev. A 79, 053824.

Acernese, F., et al., 2010a, Astropart. Phys. 33, 131.

Acernese, F., et al., 2010b, Astropart. Phys. 33, 182.

Acernese, F., et al., 2010c, Astropart. Phys. 33, 75.

Ade, P. A. R., et al. (Planck Collaboration), 2013, arXiv:1303.5062.

Adhikari, R., 2004, Sensitivity and Noise Analysis of $4 \mathrm{~km}$ Laser Interferometric Gravitational Wave Antennae, Ph.D. thesis (Massachusetts Institute of Technology) [http://hdl.handle.net/ 1721.1/28646].

Ageev, A., B. C. Palmer, A. D. Felice, S. D. Penn, and P. R. Saulson, 2004, Classical Quantum Gravity 21, 3887.

Ajith, P., et al., 2012, Classical Quantum Gravity 29, 124001.

Aki, K., and P.G. Richards, 2009, Quantitative Seismology (University Science Books, Sausalito, CA), 2nd ed.

Allen, B., 1988, Phys. Rev. D 37, 2078.

Allen, B., 1997, in Relativistic Gravitation and Gravitational Radiation, edited by J.-A. Marck, and J.-P. Lasota (Cambridge University Press, Cambridge, England), p. 373.

Allen, B., W. G. Anderson, P. R. Brady, D. A. Brown, and J. D. E. Creighton, 2012, Phys. Rev. D 85, 122006.

Ando, M., K. Ishidoshiro, K. Yamamoto, K. Yagi, W. Kokuyama, K. Tsubono, and A. Takamori, 2010, Phys. Rev. Lett. 105, 161101. Ando, M., et al., 2010, Classical Quantum Gravity 27, 084010.

Ando, S., et al., 2013, Rev. Mod. Phys. 85, 1401.

Andre, P., et al. (PRISM Collaboration), 2013, arXiv:1306.2259.

Anholm, M., S. Ballmer, J.D.E. Creighton, L.R. Price, and X. Siemens, 2009, Phys. Rev. D 79, 084030.

Arai, K., et al., 2009, Classical Quantum Gravity 26, 204020.

Arai, K., and TAMA Collaboration, 2002, Classical Quantum Gravity 19, 1843.

Arain, M. A., and G. Mueller, 2008, Opt. Express 16, 10018.

Armstrong, J.W., 2006, Living Rev. Relativity 9, 1 [http:// www.livingreviews.org/lrr-2006-1].

Armstrong, J. W., F. B. Estabrook, and M. Tinto, 1999, Astrophys. J. 527, 814.

Armstrong, J. W., L. Iess, P. Tortora, and B. Bertotti, 2003, Astrophys. J. 599, 806.

Asmar, S. W., J. W. Armstrong, L. Iess, and P. Tortora, 2005, Radio Sci. 40, RS2001.

Aso, Y., and A. Araya, 2012, Kamioka seismic data (private communication).

Aston, S. M., et al., 2012, Classical Quantum Gravity 29, 235004.

Aufmuth, P., and K. Danzmann, 2005, New J. Phys. 7, 202.

Augst, S. J., and R. W. P. Drever, 2000, AIP Conf. Proc. 523, 338.

Ballardin, G., et al., 2001, Rev. Sci. Instrum. 72, 3643.

Ballmer, S.W., 2006, LIGO interferometer operating at design sensitivity with application to gravitational radiometry, $\mathrm{Ph} . \mathrm{D}$. thesis (Massachusetts Institute of Technology).

Balser, M., and C. A. Wagner, 1960, Nature (London) 188, 638.

Barish, B., and R. Weiss, 1999, Phys. Today 52, No. 10, 44.

Barkhausen, H., 1919, Phys. Z. 20, 401.

Barsotti, L., 2006, The control of the Virgo interferometer for gravitational wave detection, Ph.D. thesis (University of Pisa).

Barsotti, L., M. Evans, and P. Fritschel, 2010, Classical Quantum Gravity 27, 084026.

Bass, M., and V. Mahajan, 2009, Handbook of Optics: Geometrical and Physical Optics, Polarized Light, Components and Instruments (McGraw-Hill, New York), Vol. 1 [http:// books.google.com/books?id=xmDntwAACAAJ].
Bassiri, R., K. Evans, K. Borisenko, M. Fejer, J. Hough, I. MacLaren, I. Martin, R. Route, and S. Rowan, 2013, Acta Mater. 61, 1070.

Baumann, D., A. Cooray, S. Dodelson, J. Dunkley, A. A. Fraisse, M. G. Jackson, A. Kogut, L. M. Krauss, K. M. Smith, and M. Zaldarriaga, 2009, in A Mission to Map our Origins, edited by S. Dodelson, D. Baumann, A. Cooray, J. Dunkley, A. Fraisse, M. G. Jackson, A. Kogut, L. Krauss, M. Zaldarriaga, and K. Smith, AIP Conf. Proc. No. 1141 (AIP, New York), pp. 3-9 [http:// adsabs.harvard.edu/abs/2009AIPC.1141....3B].

Beccaria, M., et al., 1998, Classical Quantum Gravity 15, 3339.

Beker, M. G., et al., 2011, Gen. Relativ. Gravit. 43, 623.

Belczynski, K., V. Kalogera, and T. Bulik, 2002, Astrophys. J. 572, 407.

Bender, P. L., 2011, Phys. Rev. D 84, 028101.

Bender, P. L., 2012, Gen. Relativ. Gravit. 44, 711.

Bennett, C. L., et al., 2012, arXiv:1212.5225.

Bernard, W., and H. B. Callen, 1959, Rev. Mod. Phys. 31, 1017.

Bertotti, G., 1998, Hysteresis in Magnetism: For Physicists, Materials Scientists, and Engineers, Electromagnetism Series (Academic Press, New York) [http://books.google.com/books? $\mathrm{id}=\mathrm{B} 7 \mathrm{xZctzCPfgC}]$.

Beyersdorf, P.T., R.L. Byer, and M.M. Fejer, 2000, AIP Conf. Proc. 523, 200.

Bildsten, L., 1998, Astrophys. J. Lett. 501, L89.

Bittel, H., 1969, IEEE Trans. Magn. 5, 359.

Blair, D., E. Howell, L. Ju, and C. Zhao, 2012, Advanced Gravitational Wave Detectors (Cambridge University Press, Cambridge, England) [http://books.google.com/books? id $=$ mvVBkgQZrecC].

Bochner, B., 2003, Gen. Relativ. Gravit. 35, 1029.

Borselli, M., T. J. Johnson, and O. Painter, 2006, Appl. Phys. Lett. 88, 131114.

Brady, P. R., T. Creighton, C. Cutler, and B. F. Schutz, 1998, Phys. Rev. D 57, 2101.

Braginsky, V. B., 2008, Astron. Lett. 34, 558.

Braginsky, V. B., M. L. Gorodetsky, F. Y. Khalili, and K. S. Thorne, 2000, Phys. Rev. D 61, 044002.

Braginsky, V. B., and F. Y. Khalili, 1996, Rev. Mod. Phys. 68, 1.

Braginsky, V.B., and F. Y. Khalili, 1999, Quantum Measurement (Cambridge University Press, Cambridge, England).

Braginsky, V.B., O. G. Ryazhskaya, and S.P. Vyatchanin, 2006, Phys. Lett. A 359, 86.

Braginsky, V. B., S.E. Strigin, and S.P. Vyatchanin, 2001, Phys. Lett. A 287, 331.

Braginsky, V.B., S.E. Strigin, and S.P. Vyatchanin, 2002, Phys. Lett. A 305, 111.

Braginsky, V.B., and S.P. Vyatchanin, 2003, Phys. Lett. A 312, 244.

Braginsky, V. B., and S. P. Vyatchanin, 2004, Phys. Lett. A 324, 345.

Brooks, A. F., et al., 2009, Appl. Opt. 48, 355.

Brown, B. L., A. P. Mills, and J. A. Tyson, 1982, Phys. Rev. D 26, 1209.

Brown, D., D. Friedrich, F. Brückner, L. Carbone, R. Schnabel, and A. Freise, 2013, Opt. Lett. 38, 1844.

Brückner, F., T. Clausnitzer, O. Burmeister, D. Friedrich, E.-B. Kley, K. Danzmann, A. Tünnermann, and R. Schnabel, 2008, Opt. Lett. 33, 264.

Bunkowski, A., O. Burmeister, D. Friedrich, K. Danzmann, and R. Schnabel, 2006, Classical Quantum Gravity 23, 7297.

Buonanno, A., and Y. Chen, 2001, Phys. Rev. D 64, 042006.

Buonanno, A., and Y. Chen, 2002, Phys. Rev. D 65, 042001.

Cagnoli, G., L. Gammaitoni, J. Kovalik, F. Marchesoni, and M. Punturo1999, Phys. Lett. A 255, 230. 
Cagnoli, G., J. Hough, D. DeBra, M. Fejer, E. Gustafson, S. Rowan, and V. Mitrofanov, 2000, Phys. Lett. A 272, 39.

Cagnoli, G., and P. A. Willems, 2002, Phys. Rev. B 65, 174111.

Caldwell, R., M. Kamionkowski, and L. Wadley, 1998, Phys. Rev. D 59, 027101.

Callen, H. B., and T. A. Welton, 1951, Phys. Rev. 83, 34.

Camp, J. B., H. Yamamoto, S. E. Whitcomb, and D. E. McClelland, 2000, J. Opt. Soc. Am. A 17, 120.

Campbell, W.H., 1965, in Report on Equatorial Aeronomy, edited by F. de Mendonça (National Academies, Washington, DC), p. 495.

Caves, C. M., 1981, Phys. Rev. D 23, 1693.

Caves, C. M., and B.L. Schumaker, 1985, Phys. Rev. A 31, 3068.

Cella, G., 2006, Underground Reduction of Gravity Gradient Noise, Gravitational Wave Advanced Detector Workshop [https:// dcc.ligo.org/LIGO-G060311/public].

Cella, G., and A. Giazotto, 2006, Phys. Rev. D 74, 042001.

Cella, G., and A. Giazotto, 2011, Rev. Sci. Instrum. 82, 101101.

Chakrabarty, D., E.H. Morgan, M. P. Muno, D. K. Galloway, R.

Wijnands, M. van der Klis, and C. B. Markwardt, 2003, Nature (London) 424, 42.

Chapline, G. F., J. Nuckolls, and L. L. Wood, 1974, Phys. Rev. D 10, 1064.

Chelkowski, S., S. Hild, and A. Freise, 2009, Phys. Rev. D 79, 122002.

Chen, Y., 2003, Phys. Rev. D 67, 122004.

Chen, Y., 2013, arXiv:1302.1924.

Chen, Y., S.L. Danilishin, F. Y. Khalili, and H. Müller-Ebhardt, 2011, Gen. Relativ. Gravit. 43, 671.

Christensen, N., 1992, Phys. Rev. D 46, 5250.

Chua, S. S. Y., M. S. Stefszky, C. M. Mow-Lowry, B. C. Buchler, S. Dwyer, D. A. Shaddock, P. K. Lam, and D. E. McClelland, 2011, Opt. Lett. 36, 4680.

Cole, G. D., S. Groblacher, K. Gugler, S. Gigan, and M. Aspelmeyer, 2008, Appl. Phys. Lett. 92, 261108.

Corbitt, T., and N. Mavalvala, 2004, J. Opt. B 6, S675.

Corbitt, T., D. Ottaway, E. Innerhofer, J. Pelc, and N. Mavalvala, 2006, Phys. Rev. A 74, 021802.

Cornish, N. J., and E. K. Porter, 2007, Phys. Rev. D 75, 021301.

Creighton, T., 2008, Classical Quantum Gravity 25, 125011.

Cumming, A., A. Heptonstall, R. Kumar, W. Cunningham, C. Torrie, M. Barton, K. A. Strain, J. Hough, and S. Rowan, 2009, Classical Quantum Gravity 26, 215012.

Cumming, A. V., et al., 2012, Classical Quantum Gravity 29, 035003.

Cutler, C., and J. Harms, 2006, Phys. Rev. D 73, 042001.

Cutler, C., and D.E. Holz, 2009, Phys. Rev. D 80, 104009.

Cutler, C., and K. S. Thorne, 2002, arXiv:gr-qc/0204090.

D'Ambrosio, E., R. O'Shaugnessy, K. Thorne, P. Willems, S. Strigin, and S. Vyatchanin, 2004, Classical Quantum Gravity 21, S867.

Danzmann, K., 2013, The Gravitational Universe, Technical Report, Albert Einstein Institute Hannover [https://www.elisascience.org/ whitepaper/].

Danzmann, K., and A. Rüdiger, 2003, Classical Quantum Gravity 20, S1.

Daw, E. J., J. A. Giaime, D. Lormand, M. Lubiński, and J. Zweizig, 2004, Classical Quantum Gravity 21, 2255.

DECIGO, 2011, http://tamago.mtk.nao.ac.jp/decigo/.

Demeester, P., I. Pollentier, P. D. Dobbelaere, C. Brys, and P. V. Daele, 1993, Semicond. Sci. Technol. 8, 1124.

DeRosa, R., J. C. Driggers, D. Atkinson, H. Miao, V. Frolov, M. Landry, J.A. Giaime, and R.X. Adhikari, 2012, Classical Quantum Gravity 29, 215008.
Detweiler, S., 1979, Astrophys. J. 234, 1100.

de Vine, G., B. Ware, K. McKenzie, R. E. Spero, W. M. Klipstein, and D. A. Shaddock, 2010, Phys. Rev. Lett. 104, 211103.

Dimopoulos, S., P. W. Graham, J. M. Hogan, M. A. Kasevich, and S. Rajendran, 2008, Phys. Rev. D 78, 122002.

Dimopoulos, S., P. W. Graham, J. M. Hogan, M. A. Kasevich, and S. Rajendran, 2011, Phys. Rev. D 84, 028102.

Dooley, K., 2011, Design and Performance of High Laser Power Interferometers for Gravitational-wave Detection, Ph.D. thesis (University of Florida).

Douglass, D. H., R. Q. Gram, J. A. Tyson, and R.W. Lee, 1975, Phys. Rev. Lett. 35, 480.

Drever, R.W.P., 1983a, in Lecture Notes in Physics (SpringerVerlag, Berlin), Vol. 124, pp. 321-338.

Drever, R. W. P., 1983b, AIP Conf. Proc. 96, 336.

Drever, R.W.P., 1991, in The Detection of Gravitational Waves, edited by D. G. Blair (Cambridge University Press, Cambridge, England), p. 306.

Drever, R.W.P., 1996, in Dark Matter in Cosmology Quantam Measurements Experimental Gravitation, edited by R. Ansari, Y. Giraud-Heraud, and J. Tran Thanh Van (Atlantica Séguier Frontières, France), p. 375.

Drever, R. W. P., J. L. Hall, F. V. Kowalski, J. Hough, G. M. Ford, A. J. Munley, and H. Ward, 1983, Appl. Phys. B 31, 97.

Driggers, J. C., M. Evans, K. Pepper, and R. Adhikari, 2012, Rev. Sci. Instrum. 83, 024501.

Driggers, J. C., and J. Harms, 2011, Results of Phase 1 Newtonian Noise Measurements at the LIGO Sites, Technical Report No. T1100237, LIGO [https://dcc.ligo.org/LIGO-T1100237/ public].

Driggers, J. C., J. Harms, and R. X. Adhikari, 2012, Phys. Rev. D 86, 102001.

Durin, G., and S. Zapperi, 2004, arXiv:cond-mat/0404512 [http:// arxiv.org/abs/cond-mat/0404512].

Dwyer, S., 2013, Quantum noise reduction using squeezed states in LIGO, Ph.D. thesis (Massachusetts Institute of Technology) [http://hdl.handle.net/1721.1/79427].

Einstein @ Home, 2012 [http://einstein.phys.uwm.edu/].

Einstein, A., 1916, Sitzungsberichte der Königlich Preußischen Akademie der Wissenschaften (Berlin) 33, 688.

Einstein, A., 1918, Sitzungsberichte der Königlich Preußischen Akademie der Wissenschaften (Berlin) 8, 154.

Einstein, A., and A. Engel, 1997, The Collected Papers of Albert Einstein: The Berlin Years: Writings, 1914-1917 (Princeton University Press, Princeton, NJ) [http://books.google.com/ books?id=AfMclQEACAAJ].

Einstein Telescope Science Team, 2011, Einstein gravitational wave telescope conceptual design study [http://www.et-gw.eu/].

Estabrook, F. B., and H. D. Wahlquist, 1975, Gen. Relativ. Gravit. 6, 439.

Evans, K., R. Bassiri, I. Maclaren, S. Rowan, I. Martin, J. Hough, and K. Borisenko, 2012, J. Phys. Conf. Ser. 371, 012058.

Evans, M., S. Ballmer, M. Fejer, P. Fritschel, G. Harry, and G. Ogin, 2008, Phys. Rev. D 78, 102003.

Evans, M., L. Barsotti, and P. Fritschel, 2010, Phys. Lett. A 374, 665.

Faller, J., P. Bender, J. Hall, D. Hils, R. Stebbins, and M. Vincent, 1989, Adv. Space Res. 9, 107.

Farmer, A. J., and E. S. Phinney, 2003, Mon. Not. R. Astron. Soc. 346, 1197.

Faye, G., S. Marsat, L. Blanchet, and B. R. Iyer, 2012, Classical Quantum Gravity 29, 175004.

Feynman, R.P., 1965, Feynman lectures on physics, Quantum Mechanics Vol. 3 (Addison-Wesley, Reading, MA). 
Flaminio, R., J. Franc, C. Michel, N. Morgado, L. Pinard, and B. Sassolas, 2010, Classical Quantum Gravity 27, 084030.

Flanagan, E. E., and K. S. Thorne, 1995, Scattered-Light Noise for LIGO, Technical Report No. T950102, LIGO [https://dcc.ligo.org/ LIGO-T950102/public].

Fraisse, A. A., et al., 2009, in "Prospects for polarized foreground removal," edited by S. Dodelson, D. Baumann, A. Cooray, J. Dunkley, A. Fraisse, M. G. Jackson, A. Kogut, L. Krauss, M. Zaldarriaga, and K. Smith, AIP Conf. Ser. No. 1141 (AIP, New York), pp. 265-310.

Freise, A., A. Bunkowski, and R. Schnabel, 2007, New J. Phys. 9, 433.

Freise, A., and K. A. Strain, 2010, Living Rev. Relativity 13, 1 [http://www.livingreviews.org/lrr-2010-1].

Fricke, T. T., et al., 2012, Classical Quantum Gravity 29, 065005.

Friedrich, D., et al., 2011, Opt. Express 19, 14955.

Fritschel, P., R. Bork, G. González, N. Mavalvala, D. Ouimette, H. Rong, D. Sigg, and M. Zucker, 2001, Appl. Opt. 40, 4988.

Fritschel, P., N. Mavalvala, D. Shoemaker, D. Sigg, M. Zucker, and G. González, 1998, Appl. Opt. 37, 6734.

Fritschel, P., D. Shoemaker, and R. Weiss, 1992, Appl. Opt. 31, 1412.

Gaidarzhy, A., M. Imboden, P. Mohanty, J. Rankin, and B. W. Sheldon, 2007, Appl. Phys. Lett. 91, 203503.

Giaime, J., P. Saha, D. Shoemaker, and L. Sievers, 1996, Rev. Sci. Instrum. 67, 208.

Giaime, J. A., E. J. Daw, M. Weitz, R. Adhikari, P. Fritschel, R. Abbott, R. Bork, and J. Heefner, 2003, Rev. Sci. Instrum. 74, 218.

Giazotto, A., 1989, Phys. Rep. 182, 365.

Giazotto, A., 1998, Phys. Lett. A 245, 203.

Giazotto, A., 2012, Phys. Lett. A 376, 667.

Gillespie, A., and F. Raab, 1995, Phys. Rev. D 52, 577.

Goda, K., O. Miyakawa, E. E. Mikhailov, S. Saraf, R. Adhikari, K. McKenzie, R. Ward, S. Vass, A. J. Weinstein, and N. Mavalvala, 2008, Nat. Phys. 4, 472.

González, G., 2000, Classical Quantum Gravity 17, 4409.

González, G. I., and P. R. Saulson, 1994, J. Acoust. Soc. Am. 96, 207.

Goßler, S., J. Cumpston, K. McKenzie, C.M. Mow-Lowry, M.B. Gray, and D.E. McClelland, 2007, Phys. Rev. A 76, 053810.

Granata, M., M. Barsuglia, R. Flaminio, A. Freise, S. Hild, and J. Marque, 2010, J. Phys. Conf. Ser. 228, 012016.

Granata, M., C. Buy, R. Ward, and M. Barsuglia, 2010, Phys. Rev. Lett. 105, 231102.

Gras, S., D. G. Blair, and C. Zhao, 2009, Classical Quantum Gravity 26, 135012

Gretarsson, A., and G. Harry, 1999, Rev. Sci. Instrum. 70, 4081.

Gretarsson, A. M., E. D'Ambrosio, V. Frolov, B. O'Reilly, and P. K. Fritschel, 2007, J. Opt. Soc. Am. B 24, 2821.

Gretarsson, A. M., G. M. Harry, S. D. Penn, P. R. Saulson, W. J. Startin, S. Rowan, G. Cagnoli, and J. Hough, 2000, Phys. Lett. A 270, 108.

Gréverie, C., A. Brillet, C. N. Man, W. Chaibi, J.P. Coulon, and K. Feliksik, 2010, in Conference on Lasers and ElectroOptics (Optical Society of America, San Jose, CA), p. JTuD36 [http://www.opticsinfobase.org/abstract.cfm?URI=CLEO-2010JTuD36].

Grote, H., 2003, Making it Work: Second Generation Interferometry in GEO 600!, Ph.D. thesis (Universität Hannover).

Grote, H., 2008, Classical Quantum Gravity 25, 114043.

Grote, H., 2010, Classical Quantum Gravity 27, 084003.

Grote, H., G. Heinzel, A. Freise, S. Gossler, and B. Willke, 2002, Classical Quantum Gravity 19, 1849.
Hammond, G. D., A. V. Cumming, J. Hough, R. Kumar, K. Tokmakov, S. Reid, and S. Rowan, 2012, Classical Quantum Gravity 29, 124009.

Hammond, G. D., A. Pulido-Paton, C. C. Speake, and C. Trenkel, 2004, Rev. Sci. Instrum. 75, 955.

Hardham, C., et al., 2004, in Proceedings of ASPE Spring Topical Meeting on Control of Precision Systems (American Society for Precision Engineering, Raleigh, NC), pp. 127-132.

Harms, J., Y. Chen, S. Chelkowski, A. Franzen, H. Vahlbruch, K. Danzmann, and R. Schnabel, 2003, Phys. Rev. D 68, 042001.

Harms, J., R. DeSalvo, S. Dorsher, and V. Mandic, 2009, Phys. Rev. D 80, 122001.

Harms, J., B. J. J. Slagmolen, R.X. Adhikari, M. C. Miller, M. Evans, Y. Chen, H. Müller, and M. Ando, 2013, arXiv:1308.2074.

Harry, G., T. Bodiya, and R. DeSalvo, 2012, Optical Coatings and Thermal Noise in Precision Measurement (Cambridge University Press, Cambridge, England) [http://books.google.com/books? id=770n4QLmBrMC].

Harry, G. M., 2010, Classical Quantum Gravity 27, 084006.

Harry, G. M., et al., 2002, Classical Quantum Gravity 19, 897.

Harry, G. M., et al., 2006, Appl. Opt. 45, 1569.

Harry, G. M., et al., 2007, Classical Quantum Gravity 24, 405.

Hawking, S., and W. Israel, 1989, Eds., Three Hundred Years of Gravitation, Philosophiae Naturalis, Principia Mathematica (Cambridge University Press, Cambridge, England).

Haykin, S., 2002, Adaptive Filter Theory, Prentice-Hall Information and System Sciences Series (Prentice-Hall, Englewood Cliffs, $\mathrm{NJ})$.

Hebard, A. F., 1973, Rev. Sci. Instrum. 44, 425.

Heinzel, G., K. A. Strain, J. Mizuno, K. D. Skeldon, B. Willke, W. Winkler, R. Schilling, A. Rüdiger, and K. Danzmann, 1998, Phys. Rev. Lett. 81, 5493.

Hellings, R. W., and G. S. Downs, 1983, Astrophys. J. 265, L39.

Hello, P., and J. Vinet, 1990, J. Phys. (Les Ulis, Fr.) 51, 1267.

Hello, P., and J.-Y. Vinet, 1993, Phys. Lett. A 178, 351.

Hensley, J. M., A. Peters, and S. Chu, 1999, Rev. Sci. Instrum. 70, 2735.

Heptonstall, A., et al., 2010, Classical Quantum Gravity 27, 035013. Herriott, D. R., and H. J. Schulte, 1965, Appl. Opt. 4, 883.

Hewish, A., S. J. Bell, J.D.H. Pilkington, P. F. Scott, and R. A. Collins, 1968, Nature (London) 217, 709.

Hild, S., et al., 2006, Appl. Opt. 45, 7269.

Hirose, E., K. Kawabe, D. Sigg, R. Adhikari, and P. R. Saulson, 2010, Appl. Opt. 49, 3474.

Hogan, J. M., et al., 2011, Gen. Relativ. Gravit. 43, 1953.

Hohensee, M., S.-Y. Lan, R. Houtz, C. Chan, B. Estey, G. Kim, P.-C. Kuan, and H. Müller, 2011, Gen. Relativ. Gravit. 43, 1905.

Hong, T., J. Miller, H. Yamamoto, Y. Chen, and R. Adhikari, 2011, Phys. Rev. D 84, 102001.

Hong, T., H. Yang, E. Gustafson, R. Adhikari, and Y. Chen, 2012, arXiv:1207.6145.

Hu, W., and S. Dodelson, 2002, Annu. Rev. Astron. Astrophys. 40, 171.

Hu, W., and M. White, 1997, New Astron. 2, 323.

Huang, Y., J. Benesty, and J. Chen, 2006, Acoustic MIMO Signal Processing, Signals and Communication Technology (Springer, New York).

Hughes, S. A., and K. S. Thorne, 1998, Phys. Rev. D 58, 122002.

Ipser, J. R., 1971, Astrophys. J. 166, 175.

Ishidoshiro, K., M. Ando, A. Takamori, H. Takahashi, K. Okada, N. Matsumoto, W. Kokuyama, N. Kanda, Y. Aso, and K. Tsubono, 2011, Phys. Rev. Lett. 106, 161101.

Jayawant, B. V., 1981, Rep. Prog. Phys. 44, 411.

Ju, L., D. G. Blair, and C. Zhao, 2000, Rep. Prog. Phys. 63, 1317. 
Ju, L., D. G. Blair, C. Zhao, S. Gras, Z. Zhang, P. Barriga, H. Miao, Y. Fan, and L. Merrill, 2009, Classical Quantum Gravity 26, 015002.

Kafka, R., and L. Schnupp, 1978, Astron. Astrophys. 70, 97 [http:// adsabs.harvard.edu/abs/1978A\&A....70...97K.

KAGRA, 2011[http://gwcenter.icrr.u-tokyo.ac.jp/en/].

Khalili, F. Y., 2002, Phys. Lett. A 298, 308.

Khalili, F. Y., 2007, Phys. Rev. D 76, 102002.

Kimble, H. J., Y. Levin, A. B. Matsko, K. S. Thorne, and S.P. Vyatchanin, 2001, Phys. Rev. D 65, 022002.

Kippenberg, T. J., H. Rokhsari, T. Carmon, A. Scherer, and K. J. Vahala, 2005, Phys. Rev. Lett. 95, 033901.

Knispel, B., et al., 2011, Astrophys. J. Lett. 732, L1.

Kondratiev, N. M., A. G. Gurkovsky, and M. L. Gorodetsky, 2011, Phys. Rev. D 84, 022001.

Kovalik, J., and P. R. Saulson, 1993, Rev. Sci. Instrum. 64, 2942.

Kroker, S., T. Käsebier, F. Brückner, F. Fuchs, E.-B. Kley, and A. Tünnermann, 2011, Opt. Express 19, 16466.

Kroker, S., T. Käsebier, E.-B. Kley, and A. Tünnermann, 2013, Opt. Lett. 38, 3336.

Królak, A., M. Tinto, and M. Vallisneri, 2004, Phys. Rev. D 70, 022003.

Kubo, R., 1966, Rep. Prog. Phys. 29, 255.

Kwee, P., B. Willke, and K. Danzmann, 2009, Opt. Lett. 34, 2912.

Lada, C. J., 2006, Astrophys. J. Lett. 640, L63.

Lantz, B., R. Schofield, B. O'Reilly, D. E. Clark, and D. DeBra, 2009, Bull. Seismol. Soc. Am. 99, 980.

Lawrence, R., D. Ottaway, M. Zucker, and P. Fritschel, 2004, Opt. Lett. 29, 2635.

Leibrandt, D. R., J.C. Bergquist, and T. Rosenband, 2013, arXiv:1301.0022.

Levin, Y., 1998, Phys. Rev. D 57, 659.

LIGO, 2011 [https://www.advancedligo.mit.edu/].

LIGO Scientific Collaboration, J. Abadie et al., 2011, Nat. Phys. 7, 962.

Lin, A. C., J. S. Harris, and M. M. Fejer, 2011, J. Vac. Sci. Technol. B 29, 03C120 [http://dx.doi.org/10.1116/1.3562191].

Liu, Y. T., and L. Lindblom, 2001, Mon. Not. R. Astron. Soc. 324, 1063.

Logan, J.E., J. Hough, and N. A. Robertson, 1993, Phys. Lett. A 183, 145.

Lorén-Aguilar, P., J. Guerrero, J. Isern, J. A. Lobo, and E. GarcíaBerro, 2005, Mon. Not. R. Astron. Soc. 356, 627.

Lorimer, D. R., 2008, Living Rev. Relativity 11, 8.

Loudon, R., 1981, Phys. Rev. Lett. 47, 815.

Lück, H., J. Degallaix, H. Grote, M. Hewitson, S. Hild, B. Willke, and K. Danzmann, 2008, J. Opt. A 10, 085004.

Lück, H., A. Freise, S. Goßler, S. Hild, K. Kawabe, and K. Danzmann, 2004, Classical Quantum Gravity 21, S985.

Lück, H., et al., 2006, Classical Quantum Gravity 23, S71.

Lück, H., et al., 2010, J. Phys. Conf. Ser. 228, 012012.

Magaña-Sandoval, F., R. X. Adhikari, V. Frolov, J. Harms, J. Lee, S. Sankar, P. R. Saulson, and J. R. Smith, 2012, J. Opt. Soc. Am. A 29, 1722.

Mandic, V., and A. Buonanno, 2006, Phys. Rev. D 73, 063008.

Márka, S., et al., 2002, Classical Quantum Gravity 19, 1605.

McClelland, D. E., N. Mavalvala, Y. Chen, and R. Schnabel, 2011, Laser Photonics Rev. 5, 677.

McClelland, D.E., C. M. Savage, A. J. Tridgell, and R. Mavaddat, 1993, Phys. Rev. D 48, 5475.

Mckenzie, K., 2008, Squeezing in the Audio Gravitational Wave Detection Band, Ph.D. thesis (Australian National University).
McKenzie, K., N. Grosse, W.P. Bowen, S.E. Whitcomb, M. B. Gray, D. E. McClelland, and P. K. Lam, 2004, Phys. Rev. Lett. 93, 161105.

Meers, B. J., 1988, Phys. Rev. D 38, 2317.

Melchior, P., 1983, The Tides of the Planet Earth (Pergamon Press, New York), 2nd ed.

Miao, H., Y. Chen, and R. Adhikari, 2013, unpublished.

Miller, J., M. Evans, L. Barsotti, P. Fritschel, M. MacInnis, R. Mittleman, B. Shapiro, J. Soto, and C. Torrie, 2011, Phys. Lett. A 375, 788.

Miller, J., P. Willems, H. Yamamoto, J. Agresti, and R. DeSalvo, 2008, Classical Quantum Gravity 25, 235016.

Misner, C. W., K. S. Thorne, and J. A. Wheeler, 1973, Gravitation (Freeman, San Francisco).

Mitrofanov, V., L. Prokhorov, K. Tokmakov, and P. Willems, 2004, Classical Quantum Gravity 21, S1083.

Miyakawa, O., et al., 2006, Phys. Rev. D 74, 022001.

Mizuno, J., 1995, Comparison of optical configurations for laserinterferometric gravitational-wave detectors, Ph.D. thesis (Universität Hannover and Max-Planck-Institut für Quantenoptik, Garching).

Mizuno, J., K. A. Strain, P. G. Nelson, J. M. Chen, R. Schilling, A. Rüdiger, W. Winkler, and K. Danzmann, 1993, Phys. Lett. A 175, 273.

Morrison, E., B. Meers, D. Robertson, and H. Ward, 1994a, Appl. Opt. 33, 5037.

Morrison, E., B. J. Meers, D. I. Robertson, and H. Ward, 1994b, Appl. Opt. 33, 5041.

Mueller, G., Q. Shu, R. Adhikari, D. B. Tanner, D. Reitze, D. Sigg, N. Mavalvala, and J. Camp, 2000, Opt. Lett. 25, 266.

Nawrodt, R., et al., 2010, arXiv:1003.2893.

Newell, D. B., S. J. Richman, P. G. Nelson, R. T. Stebbins, P. L. Bender, J. E. Faller, and J. Mason, 1997, Rev. Sci. Instrum. 68, 3211.

NGO, 2013, Yellow Book, Technical Report, NGO [https://lisalight.aei.mpg.de/lisa-light/pub/ScienceWorkingTeam/ YellowBook/].

Ott, C. D., 2009, Classical Quantum Gravity 26, 063001.

Ottaway, D., J. Betzwieser, S. Ballmer, S. Waldman, and W. Kells, 2006, Opt. Lett. 31, 450.

Ottaway, D. J., P. Fritschel, and S. J. Waldman, 2012, Opt. Express 20, 8329.

Owen, B. J., 2005, Phys. Rev. Lett. 95, 211101.

Owen, B. J., 2006, Classical Quantum Gravity 23, S1.

Pan, Y., 2006, arXiv:gr-qc/0608128.

Peebles, P. J. E., 1993, Principles of Physical Cosmology, Princeton series in physics (Princeton University Press, Princeton, NJ) [http://books.google.com/books?id=AmlEt6TJ6jAC].

Penn, S. D., A. Ageev, D. Busby, G. M. Harry, A. M. Gretarsson, K. Numata, and P. Willems, 2006, Phys. Lett. A 352, 3.

Penn, S. D., et al., 2003, Classical Quantum Gravity 20, 2917.

Peterson, J., 1993, Open-file report U. S. Geological Survey 93-322. Phillips, W. A., 1987, Rep. Prog. Phys. 50, 1657.

Phinney, E. S., 1991, Astrophys. J. Lett. 380, L17.

Phinney, E. S., 2003, NASA Mission Concept Study.

Pitkin, M., S. Reid, S. Rowan, and J. Hough, 2011, Living Rev. Relativity 14, 5.

Plissi, M. V., K. A. Strain, C. I. Torrie, N. A. Robertson, S. Killbourn, S. Rowan, S. M. Twyford, H. Ward, K. D. Skeldon, and J. Hough, 1998, Rev. Sci. Instrum. 69, 3055.

Poenaru, D., and W. Greiner, 1997, Experimental Techniques in Nuclear Physics (de Gruyter, Berlin) [http://books.google.com/ books?id=7yq7ZV2G5D8C].

Pohl, R. O., X. Liu, and E. Thompson, 2002, Rev. Mod. Phys. 74, 991. 
Pollack, S. E., M. D. Turner, S. Schlamminger, C. A. Hagedorn, and J. H. Gundlach, 2010, Phys. Rev. D 81, 021101.

Ponslet, E.R., and W.O. Miller, 1998, in "Coil springs with constrained-layer viscoelastic damping for passive isolation," Smart Structures and Materials 1998: Passive Damping and Isolation, edited by L.P. Davis, SPIE Conference Series Vol. 3327 (SPIE-International Society for Optical Engineering, Bellingham, WA), pp. 432-443.

Prince, T., and LISA Science Team, 2009, LISA: Probing the universe with gravitational waves.

Prince, T. A., M. Tinto, S. L. Larson, and J. W. Armstrong, 2002, Phys. Rev. D 66, 122002.

Punturo, M., et al., 2010, Classical Quantum Gravity 27, 084007.

Purdue, P., and Y. Chen, 2002, Phys. Rev. D 66, 122004.

Regehr, M.W., 1995, Ph.D. thesis (California Institute of Technology).

Rehbein, H., H. Müller-Ebhardt, K. Somiya, S. L. Danilishin, R. Schnabel, K. Danzmann, and Y. Chen, 2008, Phys. Rev. D 78, 062003.

Rehbein, H., H. Müller-Ebhardt, K. Somiya, C. Li, R. Schnabel, K. Danzmann, and Y. Chen, 2007, Phys. Rev. D 76, 062002.

Rempe, G., R. J. Thompson, H. J. Kimble, and R. Lalezari, 1992, Opt. Lett. 17, 363.

Ringler, A., and C. Hutt, 2010, Seismol. Res. Lett. 81, 972.

Robertson, N. A., et al., 2002, Classical Quantum Gravity 19, 4043.

Rocchi, A., E. Coccia, V. Fafone, V. Malvezzi, Y. Minenkov, and L. Sperandio, 2012, J. Phys. Conf. Ser. 363, 012016.

Romero, F., and H. Dehnen, 1981, Z. Naturforsch. Teil A 36, 948.

Rowan, S., J. Hough, and D. R. M. Crooks, 2005, Phys. Lett. A 347, 25.

Rubakov, V. A., M. V. Sazhin, and A. V. Veryaskin, 1982, Phys. Lett. 115B, 189.

Saccorotti, G., D. Piccinini, L. Cauchie, and I. Fiori, 2011, Bull. Seismol. Soc. Am. 101, 568.

Sathyaprakash, B., and B.F. Schutz, 2009, Living Rev. Relativity 12, 2 [http://www.livingreviews.org/lrr-2009-2].

Sathyaprakash, B., et al., 2012, Classical Quantum Gravity 29, 124013.

Sato, S., et al., 1999, Appl. Opt. 38, 2880.

Sato, S., et al., 2009, J. Phys. Conf. Ser. 154, 012040.

Saulson, P., 1994, Fundamentals of Interferometric Gravitational Wave Detectors (World Scientific, Singapore) [http:// books.google.com/books?id=4JyGQgAACAAJ].

Saulson, P. R., 1984, Phys. Rev. D 30, 732.

Saulson, P. R., 1990, Phys. Rev. D 42, 2437.

Sayed, A.H., 2003, Fundamentals of Adaptive Filtering (John Wiley \& Sons, New York).

Sazhin, M. V., 1978, Sov. Astron. 22.

Scheel, M. A., M. Boyle, T. Chu, L. E. Kidder, K. D. Matthews, and H. P. Pfeiffer, 2009, Phys. Rev. D 79, 024003.

Schiller, S., I. I. Yu, M. M. Fejer, and R. L. Byer, 1992, Opt. Lett. 17, 378.

Schilling, R., L. Schnupp, W. Winkler, H. Billing, K. Maischberger, and A. Rudiger, 1981, J. Phys. E 14, 65.

Schnabel, R., N. Mavalvala, D. E. McClelland, and P. K. Lam, 2010, Nat. Commun. 1, 121.

Schnupp, L., W. Winkler, K. Maischberger, A. Rudiger, and R. Schilling, 1985, J. Phys. E 18, 482.

Schofield, R., 2010 (private communication).

Schumaker, B. L., and C. M. Caves, 1985, Phys. Rev. A 31, 3093.

Shoemaker, D., R. Schilling, L. Schnupp, W. Winkler, K. Maischberger, and A. Rüdiger, 1988, Phys. Rev. D 38, 423.

Sidles, J. A., and D. Sigg, 2006, Phys. Lett. A 354, 167.
Siegman, A., 1986, Lasers (University Science Books, Mill Valley, CA).

Smith-Lefebvre, N., S. Ballmer, M. Evans, S. Waldman, K. Kawabe, V. Frolov, and N. Mavalvala, 2011, Opt. Lett. 36, 4365.

Somiya, K., 2011, arXiv:1111.7185.

Somiya, K., 2012, Classical Quantum Gravity 29, 124007.

Starobinskii, A. A., 1979, JETP Lett. 30, 719 [http:// adsabs.harvard.edu/abs/1979ZhPmR..30..719S].

Stefszky, M. S., C. M. Mow-Lowry, S. S. Y. Chua, D. A. Shaddock, B. C. Buchler, H. Vahlbruch, A. Khalaidovski, R. Schnabel, P. K. Lam, and D. E. McClelland, 2012, Classical Quantum Gravity 29, 145015.

Strain, K. A., 2012 (private communication).

Strain, K. A., K. Danzmann, J. Mizuno, P. G. Nelson, A. Rüdiger, R. Schilling, and W. Winkler, 1994, Phys. Lett. A 194, 124.

Strain, K. A., and B. J. Meers, 1991, Phys. Rev. Lett. 66, 1391.

Strain, K. A., et al., 2003, Appl. Opt. 42, 1244.

Strigin, S. E., and S. P. Vyatchanin, 2007, Phys. Lett. A 365, 10.

Sun, K.-X., B. Allard, S. Buchman, S. Williams, and R. L. Byer, 2006, Classical Quantum Gravity 23, S141.

Sun, K.-X., M. M. Fejer, E. Gustafson, and R. L. Byer, 1996, Phys. Rev. Lett. 76, 3053.

Sykora, D. M., and P. de Groot, 2011, in Society of Photo-Optical Instrumentation Engineers, SPIE Conference Series Vol. 8126 (SPIE-International Society for Optical Engineering, Bellingham, WA).

Szilágyi, B., L. Lindblom, and M. A. Scheel, 2009, Phys. Rev. D 80, 124010.

Takahashi, R., 2012 (private communication).

Takahashi, R., Y. Saito, M. Fukushima, M. Ando, K. Arai, D. Tatsumi, G. Heinzel, S. Kawamura, T. Yamazaki, and S. Moriwaki, 2002, J. Vac. Sci. Technol. A 25, 114036.

Takahashi, R., et al., 2008, Classical Quantum Gravity 25, 114036.

Tarallo, M. G., et al., 2007, Appl. Opt. 46, 6648.

Tatsumi, D., 2008, J. Phys. Conf. Ser. 120, 032011.

Tatsumi, D., K. Arai, and TAMA Collaboration, 2006, J. Phys. Conf. Ser. 32, 94.

Taylor, J.H., L. A. Fowler, and P.M. McCulloch, 1979, Nature (London) 277, 437.

Thorne, K.S., 1989, Light Scattering and Proposed Baffle Configuration for the LIGO, LIGO Technical Report, Caltech [http://www.ligo.caltech.edu/docs/T/T890017-00.pdf].

Thorne, K. S., and C. J. Winstein, 1999, Phys. Rev. D 60, 082001.

Thorpe, M. J., D. R. Leibrandt, T. M. Fortier, and T. Rosenband, 2010, Opt. Express 18, 18744.

Tinto, M., and S. V. Dhurandhar, 2005, Living Rev. Relativity 8, 4, http://www.livingreviews.org/lrr-2005-4.

Tomaru, T., T. Suzuki, T. Uchiyama, A. Yamamoto, T. Shintomi, C. T. Taylor, K. Yamamoto, S. Miyoki, M. Ohashi, and K. Kuroda, 2002, Phys. Lett. A 301, 215.

Turner, M. S., 1997, Phys. Rev. D 55, R435.

Tyson, J. A., and R. P. Giffard, 1978, Annu. Rev. Astron. Astrophys. 16, 521.

Uchiyama, T., D. Tatsumi, T. Tomaru, M. Tobar, K. Kuroda, T. Suzuki, N. Sato, A. Yamamoto, T. Haruyama, and T. Shintomi, 1998, Phys. Lett. A 242, 211.

Uchiyama, T., et al., 1999, Phys. Lett. A 261, 5.

Uehara, N., A. Ueda, K. Ueda, H. Sekiguchi, T. Mitake, K. Nakamura, N. Kitajima, and I. Kataoka, 1995, Opt. Lett. 20, 530.

Ugolini, D., M. Girard, G. M. Harry, and V.P. Mitrofanov, 2008, Phys. Lett. A 372, 5741.

Ushomirsky, G., C. Cutler, and L. Bildsten, 2000, Mon. Not. R. Astron. Soc. 319, 902. 
Vahlbruch, H., S. Chelkowski, K. Danzmann, and R. Schnabel, 2007, New J. Phys. 9, 371.

Vahlbruch, H., S. Chelkowski, B. Hage, A. Franzen, K. Danzmann, and R. Schnabel, 2006, Phys. Rev. Lett. 97, 011101.

Van Haasteren, R., Y. Levin, P. McDonald, and T. Lu, 2009, Mon. Not. R. Astron. Soc. 395, 1005.

Varvella, M., E. Calloni, L. D. Fiore, L. Milano, and N. Arnaud, 2004, Astropart. Phys. 21, 325.

Vinet, J.-Y., 2010, Phys. Rev. D 82, 042003.

Vinet, J.-Y., V. Brisson, and S. Braccini, 1996, Phys. Rev. D 54, 1276.

Virgo Collaboration, 2011, https://wwwcascina.virgo.infn.it/ advirgo/.

Virgo Collaboration, 2008, Appl. Opt. 47, 5853.

Virgo Collaboration, 2009, Internal Technical Document, "Advanced Virgo Baseline Design."

Virgo Collaboration, 2010, Appl. Opt. 49, 4780.

Virgo Collaboration, 2012, Advanced Virgo Technical Design Report, Technical Report [https://tds.ego-gw.it/ql/?c=8940].

Volland, H., 1995, Handbook of Atmospheric Electrodynamics (CRC Press, Boca Raton, FL), Vol. 1.

Vorvick, C., 2012 (private communication).

Vyatchanin, S. P., and A. B. Matsko, 1996, JETP 82, 1007.

Wahlquist, H., 1987, Gen. Relativ. Gravit. 19, 1101.

Walsh, C. J., A. J. Leistner, and B. F. Oreb, 1999, Appl. Opt. 38, 4790.

Webb, S., 1992, J. Acoust. Soc. Am. 92, 2141.

Weber, J., 1960, Phys. Rev. 117, 306.

Weber, J., 1970, Phys. Rev. Lett. 24, 276.

Weinberg, S., 2004, Phys. Rev. D 69, 023503.
Weiss, R., 1972, Electromagnetically Coupled Broadband Gravitational Antenna, Technical Report, Massachusetts Institute of Technology [https://dcc.ligo.org/cgi-bin/DocDB/ ShowDocument?docid=38618].

Weiss, R., 1999, Rev. Mod. Phys. 71, S187.

Wenzel, 2012 [http://www.wenzel.com/oscillators.htm].

Willemenot, E., and P. Touboul, 2000, Rev. Sci. Instrum. 71, 310.

Willke, B., et al., 2008, Classical Quantum Gravity 25, 114040.

Winkler, W., K. Danzmann, A. Rüdiger, and R. Schilling, 1991, Phys. Rev. A 44, 7022.

Winkler, W., R. Schilling, K. Danzmann, J. Mizuno, A. Rüdiger, and K. A. Strain, 1994, Appl. Opt. 33, 7547.

Wise, S., et al., 2005, Phys. Rev. Lett. 95, 013901.

Woodard, R. P., M. G. Romania, and N. C. Tsamis, 2011, Classical Quantum Gravity 28, 075013.

Woods, T. N., R. T. Wrigley, III, G. J. Rottman, and R.E. Haring, 1994, Appl. Opt. 33, 4273.

Wu, L.-A., H. J. Kimble, J.L. Hall, and H. Wu, 1986, Phys. Rev. Lett. 57, 2520.

Yagi, K., and T. Tanaka, 2010, Prog. Theor. Phys. 123, 1069.

Yamamoto, H., 2007 (private communication).

Yamamoto, K., H. Hayakawa, A. Okada, T. Uchiyama, S. Miyoki, M. Ohashi, K. Kuroda, N. Kanda, D. Tatsumi, and Y. Tsunesada, 2008, Phys. Rev. D 78, 022004.

Zucker, M.E., and S.E. Whitcomb, 1996, Proceedings of the Seventh Marcel Grossman Meeting on General Relativity (World Scientific, Hackensack, NJ).

ZygoEPO, 2011, ITM04 phasemap, Metrology Report. 